\title{
CHARACTERIZING THE PERFORMANCE OF THE PRECISION ARRAY FOR PROBING THE EPOCH OF REIONIZATION
}

\author{
Nicole E. Gugliucci \\ Charlottesville, VA
}

B.A., Lycoming College, 2005

M.S., University of Virginia, 2007

A Dissertation Presented to the Graduate

Faculty of the University of Virginia

in Candidacy for the Degree of

Doctor of Philosophy

\section{Department of Astronomy \\ University of Virginia}

August, 2012
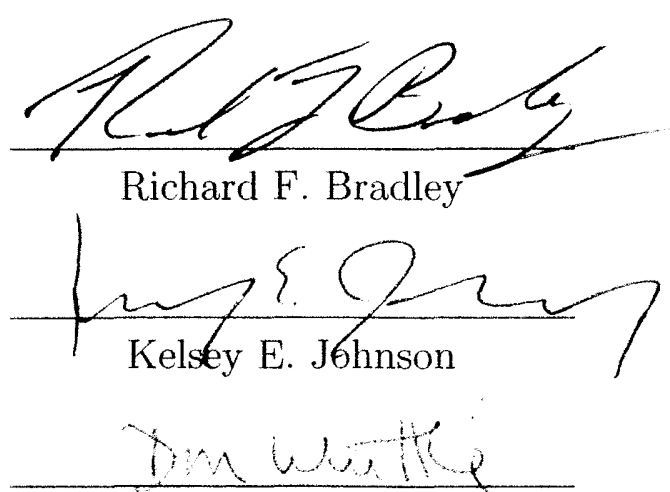

D. Mark Whittle

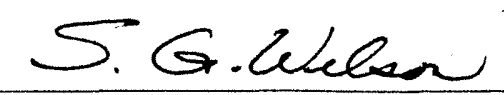

Stephen G. Wilson 


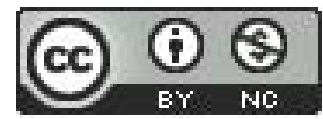

Creative Commons Attribution-NonCommericial

Nicole E. Gugliucci

August 2012 
Dedicated to "The Fabulous Four," the foundation of everything I am; and to Aunt Mary, never give up. 


\begin{abstract}
With better telescopes and observing techniques, astronomers have entered the age of precision observational cosmology. The processes of formation of the first stars and galaxies is still a mystery to us, however, as our optical and infrared telescopes are not yet powerful enough to see back to these events that occurred over 12 billion years ago in what has been coined the epoch of reionization, or EoR. The search for this signal has pushed radio astronomy to lower frequencies than have traditionally been used. This low-frequency regime is complicated by strong foreground sources, such as the emission of our own galaxy, human-made radio frequency interference, and the Earth's refractive and turbulent ionosphere.

PAPER (The Precision Array for Probing the Epoch of Reionization) is a collaboration between the University of Virginia, the National Radio Astronomy Observatory, University of California at Berkeley, University of Pennsylvania, and the Square Kilometer Array consortium in South Africa. PAPER will consist of a 128-antenna array of sleeved dipoles with a clean reception pattern over the entire frequency range of interest.

This thesis reports on work mainly done with the PAPER array in Green Bank at various stages of development. I will describe the planning of the array and various steps in the deployment and calibration process. In the early days of the experiment, it was ideal to determine the receiver temperature of the instrument in the field. This led to the discovery of a stability issue with amplifiers in the PAPER system which were then characterized to achieve the desired imaging capability.

As PAPER became more sensitive and could begin to image fainter sources on the sky, the ionosphere became more of a concern for present and future observations. The brightest sources were tracked over short timescales. After calibrating for antenna
\end{abstract}


positions, the brightest sources in the sky were shown to be changing position on the scale of arcminutes over short timescales. The implications of these findings in light of near and long-term EoR observations and other astronomical projects are discussed. 


\section{Acknowledgments}

In order to properly thank all of the people involved in the completion of this dissertation, this section would be obscenely long and far too profanity-laden for the traditional standards of academic publishing. These acknowledgments represent an abridged version. Those of you that know how much your help and support has meant to me already know it, so thank you. With any luck, the full version of acknowledgements will be available on my blog which is bound to have more readers than this dissertation ever will. But not by much.

First, I thank my advisor and mentor, Rich Bradley, without whom this crazy project would have never been done. You have been a guide and a calming force in my otherwise manic grad school career. You never let me quit, and for that I am truly grateful.

I have been incredibly fortunate to work with some of the smartest, most talented people I have ever met. Thank you to Don Backer, who left us all to soon, but whose passion for discovery kept us going through the dark times; to Aaron Parsons who has been an inspiration; to Danny Jacobs, Jonnie Pober, David Moore, James Aguirre, Chris Carilli, Jason Manley, who could beat a stake into the ground or beat the data into submission; you are all taking the radio astronomy world by storm, and I love it. To the beloved Charlottesville crew: Erin Mastrantonio, Pat Klima, Chaitali Parashare, Dan Boyd; to the fantastic support staff in Green Bank; you turned PAPER from an idea into a real, physical thing, and that is better than magic. To William Walborough, who literally drove us across the desert and back and made sure our South Africa site was equipped with everything we needed, baie dankie.

Science does not happen in a vacuum, and I was honored to share my work with 
the best astronomy grad students of all time. To my classmates, holy heckballs, we actually made it. Gail, may the astronomical community continue to confuse our identities for decades to come, as I consider it a great honor to be mistaken for you; Jarron, you let me pester you endlessly and always greeting me with humor and a smile; Jake, your guidance through my personal struggles was invaluable; Genevieve, your boundless optimism was infectious; Joleen, your professionalism was inspiring and your pies delicious; Paul, you are a perpetual motion machine of energy and goodwill; Lisa May, the cuddliest of grad students; Dan, living proof that scientists are not stuffy and boring; Rachael, a mighty force of awesome; Kristen, friend, confidante, and voice that I truly hope will lead our colleagues into the future; Tukk and Anya, my sisters in astronomy and in dance; George, who lives life to the fullest.

Many thanks to my supportive committee members who stuck with me from the proposal to the end, Mark Whittle and Kelsey Johnson. Thank you Steve Wilson for serving on my committee from the Engineering Department. And many thanks to C.C. Peterson for wading through my writing and making it actually readable!

To the science bloggers, the skeptics, and the tweeters, specifically the Space Tweeps, the Discovery News team, the Skepchicks, the Phinishers, the Bad Astronomer, and Star Stryder... you rock my world online and off.

For all the people who listened to my whining over the years, to my patient Timothy, to Mom, Paul, and Steven... I love you, and I can’t thank you enough.

This work was supported by NSF-AST Grants 0804508, 0505354, and 0607838 and the Virginia Space Grant Consortium. 


\section{Table of Contents}

ABSTRACT iv

Acknowledgments vi

List of Figures $\quad$ xiv

List of Tables $\quad$ xv

1 General Introduction 1

1.1 Epoch of Reionization . . . . . . . . . . . . . . . . . 2

1.1.1 HI Line Physics . . . . . . . . . . . . . . . . . . . . . 4

1.1.2 Temperature Effects of First Luminous Sources . . . . . . . 6

1.1.3 Reionization of the IGM: Global Signal . . . . . . . . . . . 9

1.1.4 Individual Emission Regions . . . . . . . . . . . . . . . . . 14

1.1.5 Models . . . . . . . . . . . . . . . . . . . . . 15

1.2 Radio Astronomy Fundamentals . . . . . . . . . . . . . . . . . . . 22

1.2 .1 Interferometry . . . . . . . . . . . . . . . . . . . 24

1.3 PAPER-specific Challenges . . . . . . . . . . . . . . . . . . . 28

2 PAPER Instrument 31

2.1 Hardware . . . . . . . . . . . . . . . . . . . . . . . . 31

2.2 Deployment and Configurations . . . . . . . . . . . . . . 37

2.3 Calibration and Imaging $\ldots \ldots \ldots \ldots$

3 Total Power Measurement 48

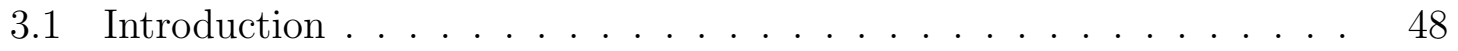

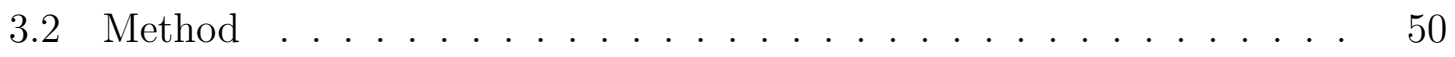

3.3 Data and Analysis . . . . . . . . . . . . . . . . . . . . 52

3.4 Engineering Work . . . . . . . . . . . . . . . . . . 55

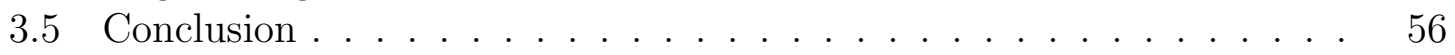


4 Effects of the Ionosphere $\quad 57$

4.1 Radio Astronomy and the Ionosphere . . . . . . . . . . . . . 57

4.2 Observations . . . . . . . . . . . . . . . . . 67

4.3 Results ........................ . . . . . 70

4.3.1 Comparison to Ionosphere Model . . . . . . . . . . . . . 80

4.4 Conclusion . . . . . . . . . . . . . . . . . . . . 82

5 General Conclusions $\quad 85$ 


\section{List of Figures}

1.1 Temperature history of the IGM without luminous sources, from Furlanetto, Oh, \& Briggs (2006), as described at the end of Section 1.1.1. The top panel shows the evolution of the CMB, kinetic, and spin temperatures. The bottom panel shows the expected signal of the brightness temperature observed in this scenario. HI is only seen in absorption in this simple model where no luminous sources are present. . . . . . .

1.2 (a) Temperatures, (b) ionization fraction, and (c) brightness temperature of the IGM with reionization dominated by Pop II stars, from Furlanetto (2006), as described above in Section 1.1.3. The solid black line represents the simple fiducial model. The red and purple dashed curves in all panels allow for feedback to slow reionization. The blue dot-dashed curves in panels (a) and (c) indicate a slower heating of the IGM. The black and blue dotted curves in panel (c) ignore shock heating. . . . . . . . . . . . . . . . .

1.3 (a) Temperatures, (b) ionization fraction, and (c) brightness temperature of the IGM with reionization dominated by Pop III stars, from Furlanetto (2006), as described above in Section 1.1.3. The solid black line represents the simple fiducial model. The red and purple dashed curves in all panels allow for feedback to slow reionization. The blue dot-dashed curves in panels (a) and (c) indicate a slower heating of the IGM. The black and blue dotted curves in panel (c) ignore shock heating. . . . . . . . . . . . . . . . . .

.4 Bubble size distribution, from Furlanetto, Zaldarriaga, \& Hernquist (2004) and as described by Equation 1.10, where each curve from left to right is for $z=18,16,14,13$, and 12. As is expected, the ionized bubbles generally grow larger at later times. . . . . . . . . . . .

1.5 Cartoon of first and second stage star formation Strömgren spheres, from Barkana and Loeb (2001). The spheres from the very first stage of star formation are thought to disappear due to reionization before the second stage is underway. . . . . . . . . . . . . . . 
1.6 Simulation of reionization from Furlanetto, Sokasian, \& Hernquist (2004), presenting nine timesteps as a result of the modeling process described above. The color from orange to red indicates the brightness temperature of the HI signal. Evolution proceeds from the first panel where the IGM is nearly completely neutral to the last panel where only filaments of neutral gas are left. . . . . . . . . . . . . . . . . . .

1.7 Milky Way synchrotron emission dominates at all but the smallest size scales (right side of the graph), where the synchrotron emission of extragalactic point sources become important. Shown here are the power spectra of various foreground contributions and of the EoR signal itself. From Santos et al. (2005). . . . . . . . . . . . . . . . .

1.8 Evolving power spectrum of the EoR, from Barkana (2009). The colored lines show the power spectrum at different ionization fractions, corresponding to different redshifts. $k$ is the wavenumber and indicates and angular scale, and $\Delta_{21}$ is the brightness temperature of the HI signal. . . . . . . . . . . . . . . .

2.1 Single PAPER element or antenna. The copper dipoles in this structure are held in place by dialectric foam and epoxy in between two precisely spaced aluminum plates. The more recent design replaces the dielectric foam with plastic spacers and is thus easier to assemble. The antenna assembly is mounted on a grey PVC pipe containing the first stage of amplification and sits on a steel and PVC groundscreen. . . . . . . .

2.2 Block diagram of individual PAPER element, as described in Section 1.3. The cosmic radiation collected at the dipole is first amplified in the balun then sent along 500-ft of RG-6 cable. The signal then enters a shielded hut or shielded boxes within a hut and is further amplified and filtered by the receivers. The signal is then digitized and correlated and sent offsite for further analysis. . . . . . . . . . . . . .

2.3 Visualization of dipole beam pattern from Computer Simulation Technology's Microwave Studio. The beam is slightly elliptical for a single dipole. The peak gain is $12.4 \mathrm{dBi}$. . . . . . . . . . . . . . .

2.4 Aerial view of the 16-antenna array in Green Bank from Google Maps, centered on the Galford Meadow. The road, central hut, and 16 groundscreens are visible. The outermost antenna in the Green Bank Three-Element Interferometer can be seen at the top left as a white circle. . . . . . . . . . . . . . . . . .

2.5 Diagram of antenna positions for PAPER-32 in Green Bank. This same minimum-redundancy configuration was later adapted for the 32-element array in South Africa. . . . . . . . . . . . . . . . 
2.6 The double-lobe structure of Centaurus A is clearly visible, along few a few point sources and the Galactic Plane, after initial calibration with the 64-element array in South Africa in July 2011. . . . . . . . . . . .

3.1 The Galactic Plane as seen at $408 \mathrm{MHz}$ by the Haslam All-Sky Survey. This image is from a HealPix map created by NASA's Legacy Archive for Microwave Background Data Analysis. . . . . . . . . . . . . . . .

3.2 Top: Model of single-antenna observations using the extrapolation of the Haslam sky map and the simulated antenna beam. Bottom: Power data from antenna 1 of 8 in Green Bank in September 2006. Both are shown as a function of Local Sidereal Time. . . . . . . . . . . . . . .

3.3 The black dashed line plots the model temperature versus the total power from the data for one antenna over a 24-hour period, matched up in time. The red solid line is the expected relationship between the model and data given the fitted set of parameters, gain and instrument temperature. . . . . . . . . . . . . . . .

3.4 Top: Model temperature and temperature predicted from the data. Bottom: Actual power data and power predicted from the model. This shows, in addition to Figure 3.3, that the fitted parameters are not precise over the entire day range. . . . . . . . . . . . . . .

3.5 Top left: Model. Top right: Data. Middle left: Result of linear fit. Middle right: Model and power extrapolated with linear fit. Bottom left: Ambient temperature with polynomial fit. Bottom right: Model with power extrapolated from fit to Eqn 3.7. . . . . . . . . . .

4.1 Electronic density as a function of elevation for daytime, nightime, solar maximum and solar minimum periods. The F2, F1, E and D layers are indicated. From Peter Guest at http://www.weather.nps.navy.mil/ psguest/ 59

4.2 Global TEC distribution from by the Australian Government Radio and Space Services as measured by GPS satellites sending signals to ground-based receivers at several frequencies. The continents are outlined in red and the color map indicates the total electron content in TECU. The area of highest density occurs on the daytime side of the Earth, following the subsolar point as ultraviolet solar radiation ionizes those regions of the atmosphere. The aforementioned equatorial anomaly can be seen as a trough in density over South America. . 
4.3 Phase of three antennas relative to a central antenna during an approximately 8 hour observation of Virgo A illustrating many of the ionospheric phenomena typically observed at the VLA. Ionospheric disturbances on small, medium, and large size scales are seen, with TID's happening during the daytime, scintillation at night, and the refractive wedge at dawn which makes observations difficult if not impossible near sunrise. From Kassim et al. (2007). . . . . . . . . . . . . . . . .

4.4 Cartoon of the four ionosphere calibration regimes. When the field of view is smaller than the scale of ionospheric density fluctuations, such as in cases (a) and (b), self-calibration is used without regard to the ionosphere. PAPER is an example of case (c) where the field of view encompasses several ionospheric patches, but the array is small in comparison to these. Larger observatories, such as LOFAR, will have to work in the most challenging regime $(\mathrm{d}) \ldots . . . . . . . .$.

4.5 Top: Position offset in arcminutes vs local sidereal time for Cas A at $130 \mathrm{MHz}$, as measured by the process described in Section 4.3. Bottom:

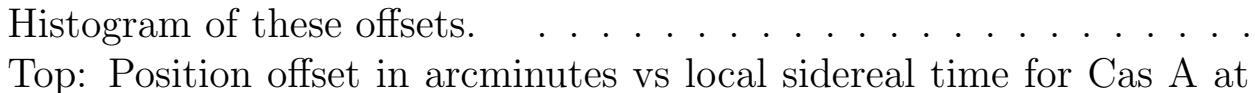
$165 \mathrm{MHz}$, as measured by the process described in Section 4.3. Bottom: Histogram of these offsets.

4.7 Top: Position offset in arcminutes vs local sidereal time for Cyg A at $130 \mathrm{MHz}$, as measured by the process described in Section 4.3. Bottom: Histogram of these offsets. . . . . . . . . . . . . . . .

4.8 Top: Position offset in arcminutes vs local sidereal time for Cyg A at $165 \mathrm{MHz}$, as measured by the process described in Section 4.3. Bottom: Histogram of these offsets.

4.9 Top: Position offset in arcminutes vs local sidereal time for Vir A at $130 \mathrm{MHz}$, as measured by the process described in Section 4.3. Bottom: Histogram of these offsets.

4.10 Top: Position offset in arcminutes vs local sidereal time for Vir A at $165 \mathrm{MHz}$, as measured by the process described in Section 4.3. Bottom: Histogram of these offsets. . . . . . . . . . . . . .

4.11 Top: Position offset in arcminutes vs local sidereal time for Tau A at $130 \mathrm{MHz}$, as measured by the process described in Section 4.3. Bottom: Histogram of these offsets. . . . . . . . . . . . . . . .

4.12 Top: Position offset in arcminutes vs local sidereal time for Tau A at $165 \mathrm{MHz}$, as measured by the process described in Section 4.3. Bottom: Histogram of these offsets. . . . . . . . . . . . . . .

4.13 Contour plot of Cygnus A made with CASA. Cyg A is an unresolved point source as seen by PAPER, and image rms 100 mJy. . . . . . . 
4.14 Results from Monte-Carlo simulations with a PAPER-like array and nine point sources using a 2-D Kolmogorev screen with a Fried length of $3 \mathrm{~km}$ at height of $300 \mathrm{~km}$. The standard deviation of position offsets are several times larger than the measured offsets in the PAPER data. 


\section{List of Tables}

1.1 PAPER Deployments . . . . . . . . . . . . . . . . . 30

4.1 Bright Sources . . . . . . . . . . . . . . . . . . . . 67

4.2 Source Position Offsets in Arcmins . . . . . . . . . . . . . . 81 


\section{Chapter 1}

\section{General Introduction}

The first astronomical observations in the radio regime were made serendipitously in the 1930s with an antenna built to receive radiation at $20.5 \mathrm{MHz}$. Karl Jansky was working for Bell Labs in New Jersey, trying to determine a source of noise for transatlantic phone service when he found a bright source that moved with the sidereal motion of the stars. This turned out to be emission from the center of the Milky Way Galaxy. The first targeted astronomical observations were made by Grote Reber several years later with his home-made radio telescope with which he detected the Milky Way at $160 \mathrm{MHz}$. Over the decades, radio astronomy moved higher in frequency to the centimeter $(\sim 1-100 \mathrm{GHz})$ regime, which was easily accessible in terms of calibration and allowed the study of a diverse array of astrophysical phenomena, from active galactic nuclei to lunar radar mapping.

Attention has returned to lower frequencies for the search for the signal of the epoch of reionization $(\mathrm{EoR})$ at redshifts $(z)$ greater than 6. Low-frequency observatories and experiments will have to contend with the refractive and often turbulent nature of the Earth's ionosphere in order to make sensitive observations. The Precision Array for Probing the Epoch of Reionization (PAPER, Parsons et al. 2010) 
is one such experiment dedicated to making the first detection of the EoR signal. The sensitive measurement must be made among foreground sources several orders of magnitude brighter that the signal itself, so a thorough understanding of these foregrounds and the effects of such observations of the ionosphere will be key to interpreting the results.

\subsection{Epoch of Reionization}

The history of the universe can be traced in part by the history of its most common atomic constituent: hydrogen. The Big Bang Theory, the accepted model of the creation of the universe, successfully predicts that the universe was first made of $75 \%$

hydrogen by mass, $25 \%$ helium, and trace amounts of lithium. These conditions were set in the first three minutes of the universe's existence when these atomic nuclei, or the protons and neutrons, were formed. Only some time later, when the universe was 380,000 years old did the conditions become cool enough for neutral atoms to be formed from protons, neutrons, and electrons.

The Cosmic Microwave Background (CMB) is the key observable of this latter event, known as recombination. This microwave radiation is detected nearly isotropically around the sky and was created by photons that were first able to escape the blinding fog of charged particles that had dominated the universe up until that point. The detection of the CMB by Penzias and Wilson in 1964 was an important confirmation of the hot Big Bang model and represents the most distant light that can ever be observed.

At this point in the Universe's history, it enters what is known as the "Dark Ages." Little is known about this epoch when the very first stars, black holes, and, eventually, galaxies came to form. 
With the development of more sensitive optical and infrared telescopes, such as the James Webb Space Telescope (JWST), the first epoch of galaxy formation will be probed in the coming decades. However, the first look will come from radio telescopes that will directly observe the intergalactic hydrogen gas as it is affected by these first galaxies in what is known as the Epoch of Reionization.

Atomic hydrogen has a hyperfine transition at $1.4 \mathrm{GHz}$. This means that the spins of the electron and proton of a hydrogen atom can be aligned or anti-aligned, and the former state is slightly more energetic than the latter. When electron "flips" from the higher state to the lower, the atom emits a photon with a wavelength of $21 \mathrm{~cm}$, or $1.4 \mathrm{GHz}$. This means that, on average, it takes over ten million years for a given atom of hydrogen to undergo this transition. Fortunately, there is a huge mass of neutral hydrogen in the universe and in the intergalactic medium (IGM).

Optical and infrared surveys have slowly been pushing the limits to the farthest galaxies and quasars at higher and higher redshifts. The Lyman- $\alpha$ forest, as measured along the lines of sight to distant quasars, probes the neutral hydrogen content through time. The Gunn-Peterson trough is the complete absorption of Ly- $\alpha$ along the line of sight to distant quasars (Gunn \& Peterson 1965). The first G-P trough was discovered by Becker et al. (2001) for a quasar with $z=6.28$ and for several more galaxies shortly after (Fan et al. 2006). However, only a tiny amount of hydrogen (neutral fraction $\sim 10^{-3}$ ) is necessary to be optically thick for this effect, so this only probes the end of reionization. Also, these galaxies and quasars only represent a few pierce points, not complete probes, so a global picture is needed.

The CMB radiation also provides information about the EoR. It originates from a redshift of 1089, and so it has to travel through the IGM, probing the ionization history of the universe. The CMB photons undergo Thompson scattering due to free 
electrons and thus probe the optical depth due to such electrons. This polarized CMB signal probes the summed history of reionization, but does not give any temporal information. The seven-year Wilkinson Microwave Anisotropy Probe data indicate $z \sim 10.5$ as the redshift for instantaneous reionization (Larson et al. 2011). Since the quasar data indicate an end of reionization around $z$ of 6 , this shows that reionization was not instantaneous. The quasar and CMB data together thus indicate a protracted history of reionization from $z \sim 6$ to 15 . This is why the HI signal from the EoR is expected to appear in a range $\sim 100-200 \mathrm{MHz}$.

\subsubsection{HI Line Physics}

Neutral hydrogen has a hyperfine transition due to the spin alignment of the proton and electron. The excitation temperature for this transition is called the spin temperature, $T_{S}$, and measures the relative number densities of atoms in the high and low energy states. The brightness temperature of the emission is

$$
T_{b}(\nu)=T_{S}\left(1-e^{-\tau_{\nu}}\right)+T_{C M B}(\nu) e^{-\tau_{\nu}},
$$

which depends on the optical depth, $\tau_{\nu}$, and the temperature of the background, which in this case is the CMB temperature as a function of redshift.

The optical depth is the integral of the absorption coefficient along the line of sight. It is described by

$$
\tau_{\nu}=\frac{3 c^{2} A_{10}}{8 \pi \nu^{2}} \int d s\left(1-e^{-E_{10} / k_{B} T_{S}}\right) \phi(\nu) n_{0}
$$

where $\nu$ is the frequency at rest, $c$ is the speed of light, $A_{10}$ is the spontaneous emission coefficient of the transition at $2.85 \times 10^{-15} \mathrm{~s}^{-1}, E_{10}$ is the energy of the tran- 
sition at $5.9 \times 10^{-6} \mathrm{eV}, k_{B}$ is Boltzmann's constant, $\phi(\nu)$ is the line profile, and $n_{0}$ is the number density of hydrogen atoms in the lower state of the hyperfine transition. The line broadening is dominated by the Hubble flow, and the number density of hydrogen is dependent on the fractional overdensity of baryons. Under these conditions, the optical depth is in the optically thin regime.

The signal to detect is the difference between the emission of the neutral hydrogen and the emission of the background CMB which is, when corrected for redshift and assuming low optical depth,

$$
\delta T_{b}(\nu) \simeq \frac{T_{S}-T_{C M B}(z)}{1+z} \tau_{\nu}
$$

Clearly, the interplay between the CMB temperature and spin temperature are important to observing the epoch of reionization. The gas kinetic temperature, $T_{K}$, is also vital to the description of the temperature evolution.

In the period of the dark ages after recombination, the IGM gas cools due to the expansion of the universe and energy conservation. However, Compton scattering of $\mathrm{CMB}$ photons by residual free electrons in the IGM heat the gas to the same temperature as the CMB, which is falling with redshift. Thus, $T_{K} \rightarrow T_{C M B} \propto$ $(1+z)$. The gas may decouple from the CMB when there are not enough free electrons available for heating due to continued recombinations. Models have shown this to happen by $z \sim 200$ (Seager et al. 1999), so the gas cools adiabatically beyond this point, or $T_{K} \propto(1+z)^{2}$. Atomic collisions allow the spin temperature to become equal to the kinetic temperature $\left(T_{S} \rightarrow T_{K}\right)$, so the spin temperature is also cooling faster than the CMB. Thus, the neutral hydrogen may be seen in absorption against the CMB at $z \sim 100$.

The spin temperature is not only dependent on the kinetic temperature; $T_{S}$ is also 
dependent on the absorption of and stimulated emission by CMB photons and by scattering of UV photons. Collisional coupling is driven mainly by collisions between hydrogen atoms, so $T_{S}=T_{K}$ for the early IGM history $(z>100)$ as above. As the universe expands, these collisions become less and less frequent. Thus, $T_{S}$ approaches $T_{C M B}$ at $z \leq 70$ according to simulations (Furlanetto et al. 2006), and $T_{S}=T_{C M B}$ at $z \sim 30$. Now, the IGM is invisible before reionization (see Figure 1.1). Some mechanism is needed to raise $T_{S}$ above $T_{C M B}$ in order for the ionization regions around the first sources to be seen as holes in HI emission.

The collisional coupling to the gas temperature and radiative coupling to the CMB are typical effects in determining the excitation temperature of transitions. For the spin temperature of HI, there is also a dependence on the Ly $\alpha$ background through a mechanism known as the Wouthuysen-Field effect (hereafter WF effect; Wouthuysen 1952; Field 1959). The absorption of a Ly- $\alpha$ photon allows a transition from the $S_{1 / 2}$ state to either the $P_{1 / 2}$ or $P_{3 / 2}$ state. After spontaneous emission of that photon, the

electron can then go back to the original hyperfine sub-level of the $S_{1 / 2}$ state, or it can flip to the other hyperfine state. In fact, any Lyman series photon can be absorbed then re-emitted, causing a spin-flip change in the process. This transition has its own excitation temperature, called the color temperature, $T_{C}$.

This process is dependent on the specific intensity of the Lyman- $\alpha$ radiation which is unknown for the high redshift universe. The evolution of $T_{K}$ from this point also depends on the populations and types of the first luminous sources.

\subsubsection{Temperature Effects of First Luminous Sources}

Up to this point, the kinetic gas temperature has decoupled from the CMB temperature in the dark universe, and the HI spin temperature was first affected by the 


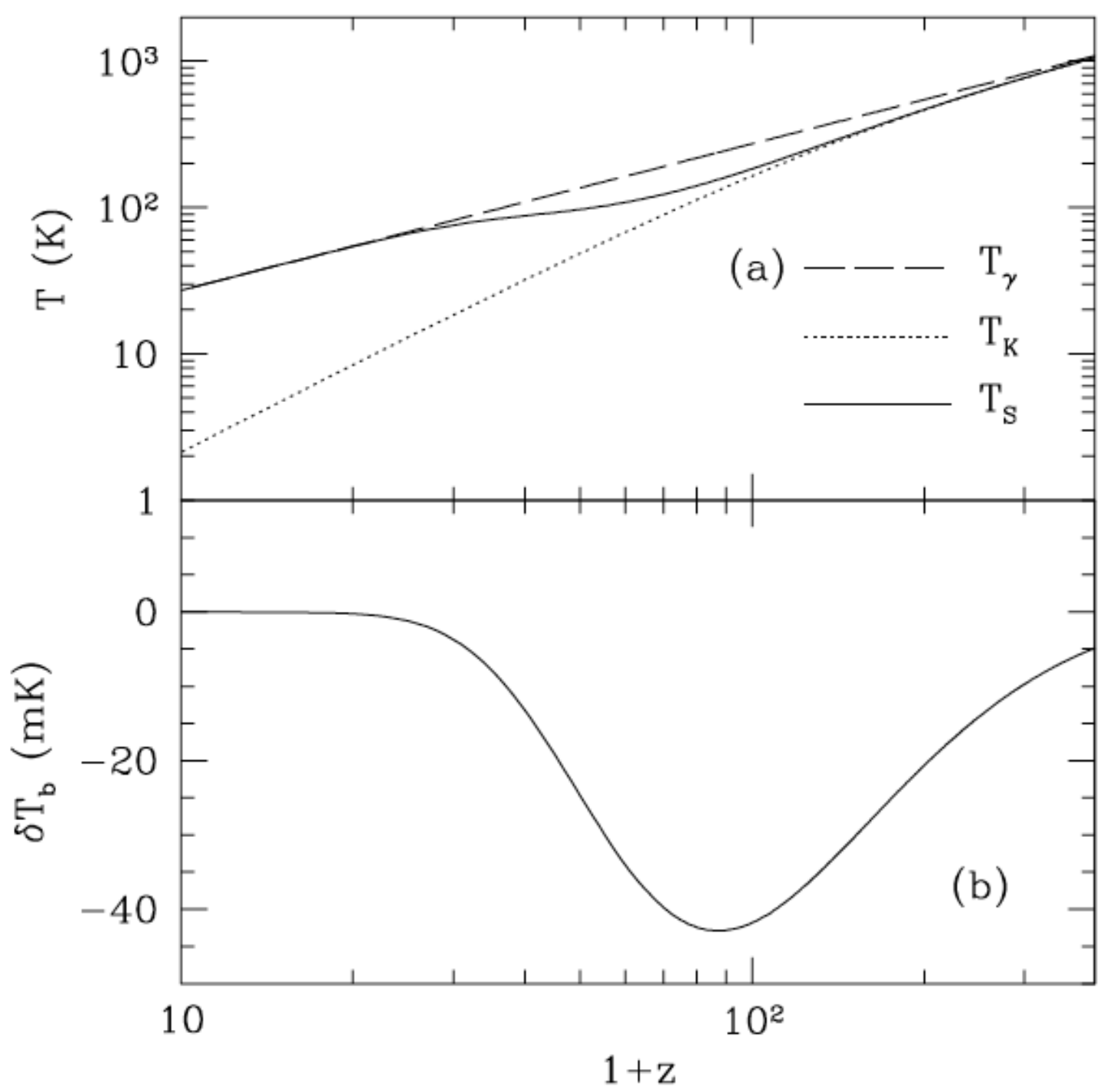

Fig. 1.1.- Temperature history of the IGM without luminous sources, from Furlanetto, Oh, \& Briggs (2006), as described at the end of Section 1.1.1. The top panel shows the evolution of the CMB, kinetic, and spin temperatures. The bottom panel shows the expected signal of the brightness temperature observed in this scenario. HI is only seen in absorption in this simple model where no luminous sources are present. 
kinetic temperature. Then, as the universe became less dense, became equal again to the CMB temperature. Thus, at $z \simeq 30, T_{K}<T_{S}=T_{C M B}$ and the IGM is once again invisible.

At some redshift, the specifics of which are dependent on the radiation field and thus the star formation rate, initial mass function of the first stars, and properties of the first $\mathrm{x}$-ray sources which may also provide Ly- $\alpha$ photons, the spin temperature will be coupled to the color temperature. The global spin temperature will drop below the CMB temperature and again become equal to the kinetic temperature. The exact redshift at which this happens depends on whether the stars involved at this time are primarily the metal-free Population III stars or the later generation of Population II stars of low metallicity. The WF effect only includes photons between Ly- $\alpha$ and the Lyman limit. Pop III stars are expected to have a number density, or $N_{\alpha}=4800$ photons per baryon with a flat spectrum in these limits. Pop II stars have $N_{\alpha}=9690$ photons per baryon with a steeply falling spectrum. Thus, the redshift at which $T_{S} \rightarrow T_{K}$ is affected strongly by the type of sources available.

When the spin temperature is once again coupled to the gas temperature via the WF effect, gas heating by luminous sources and nonlinear structure formation is taken into account . X-rays are an efficient heating source when they ionize hydrogen or helium to produce free electrons with kinetic temperatures $\sim 10^{6} \mathrm{~K}$. These free electrons distribute their energy through collisional excitations and ionizations of other atoms or through Coulomb collisions with other free electrons. The x-ray background at high redshifts is estimated by extrapolating the current x-ray background to high redshifts, but this is fraught with uncertainty. X-ray emission is most likely to come from supernovae, high-mass x-ray binaries, and active galactic nuclei, so formulas relating x-ray luminosity to star formation may be used with a correction factor, $f_{X}$, 
to take into account differences at high redshift, such that

$$
L_{X}=3.4 \times 10^{40} f_{X}\left(\frac{S F R}{1 \mathrm{M}_{\odot} \mathrm{yr}^{-1}}\right) \mathrm{erg} \mathrm{s}^{-1}
$$

(Furlanetto et al. 2006). In this formulation, the x-ray heating is rapid as long as the unknown factor is not too small, or $f_{X} \geq 0.1$.

The IGM may also be heated by shocks caused by the first nonlinear structures. It has been shown in the Millennium simulation (Springel et al. 2005) and others that the dark matter distribution of the universe is a web of sheets and filaments. These sheets form shocks if the kinetic temperature of the shock is greater than the kinetic temperature of the gas. With $T_{K, g a s} \sim 10 \mathrm{~K}$, this scenario is not difficult to achieve. More detailed simulations indicate that shocks are significant for structure formation, but are unimportant to heating in comparison to x-rays (Furlanetto et al. 2006).

A redshift at which $T_{K}$ becomes greater than $T_{C M B}$ can be defined through these processes. Since $T_{S}$ is coupled to $T_{K}$, this means that $T_{S}>T_{C M B}$, and finally the neutral hydrogen can be seen in emission in front of the CMB. Now, the stage is set for reionization to be observed.

\subsubsection{Reionization of the IGM: Global Signal}

One of the first results to come from reionization will most likely be a measure of the global ionized fraction, $\bar{x}_{i}$, as measured by the global signal as a function of redshift. In a very simple model, this is related to the global star formation rate $(S)$ such that

$$
\frac{d \bar{x}_{i}}{d t}=\zeta(z) \frac{d f_{\text {coll }}}{d t}-\alpha_{A} C \bar{x}_{i}(z) \bar{n}_{e}(z) S
$$

where $\zeta(z)$ is the ionizing efficiency in units of ionizing photons per collapsed 
atom, $f_{\text {coll }}$ is the collapsed halo fraction, $\alpha_{A}$ is the recombination coefficient, $C$ is a clumping factor due to large scale structures, and $\bar{n}_{e}(z)$ is the average electron density (Furlanetto et al. 2006). Note that most of these parameters are estimated or taken from pre-existing models of structure formation. These are set such that ionization will span the timescale $12>z>6$ as is consistent with quasar spectra and WMAP polarization results. This requires significant recombination to slow reionization that would otherwise be completed rapidly by Pop III stars.

The optical depth of the hyperfine transition and brightness temperature is used to see how the ionizing fraction is measured by the neutral hydrogen brightness temperature, such that

$$
\delta T_{b} \propto\left(\frac{T_{S}-T_{C M B}(z)}{T_{S}}\right)(1+z)\left(1-x_{i}\right)
$$

When expressed with fiducial parameters, this is

$$
\delta T_{b}=27\left(1-x_{i}\right)(1+\delta)\left(\frac{\Omega_{b} h^{2}}{0.023}\right)\left(\frac{0.15}{\Omega_{m} h^{2}} \frac{1+z}{10}\right)^{1 / 2}\left(\frac{T_{S}-T_{C M B}(z)}{T_{S}}\right) \mathrm{mK}
$$

where $\delta$ is the fractional overdensity, $\Omega_{b}$ is the ratio of the density of baryons to the critical density, $\Omega_{m}$ is the ratio of the density of matter to the critical density, and $h$ is the dimensionless Hubble parameter. Furlanetto et al. (2006) go through the analytic calculations outlined here to produce Figure 1.2 for Pop II parameters for the ionizing sources. The solid lines represent this simple model in all three panels, and reionization is complete at $z \sim 7$. The dashed curves in panel (b) allow for feedback to slow reionization, matching observational constraints. Panel (c) shows the most interesting constraints for future observations, where the absorption is strong 
$\left(\left|\delta T_{b}\right|_{\max } \sim 80 \mathrm{mK}\right)$ before reionization, and emission levels reach $30 \mathrm{mK}$ at the beginning of reionization $(z \sim 12)$ and decline during reionization. Fortunately, the dimming of emission is delayed as reionization is delayed in the models with feedback. The dot-dashed curves in panels (a) and (c) indicate a slower heating of the IGM. This allows for a stronger absorption signal and weaker emission against the CMB background, making reionization harder to detect. Finally, the dotted curves in panel (c) ignore shock heating, and this has little effect.

If the epoch of reionization is dominated by Pop III stars, then our parameters change so that reionization proceeds as in Figure 1.3. Again, reionization ends at $z \sim 7$ without feedback. Since Pop III stars are more efficient at heating than Pop II stars, the redshift at which the gas temperature rises above the CMB temperature is closer to the redshift at which the spin temperature couples to the gas temperature than for Pop II. Therefore, the absorption era is shorter and the signal weaker than previously seen. The emission is also weaker during the epoch of reionization, so a high abundance of Pop III stars at this time will make EoR harder to detect. For the long-dashed line, the escape fraction is unity and ionization happens quickly, by $z \sim 11$, so it is only visible in the absorption epoch. The short-dashed curve allows for very efficient $\mathrm{x}$-ray production, increasing the heating rate and making the absorption epoch hardly observable, but ionization is still seen during the emission epoch. The combination of these constraints is the worst-case scenario for detecting the global signal of reionization, presented as the dot-dashed line. The signal only peaks at $\delta T_{b} \leq 10 \mathrm{mK}$

Though one might expect the global sky signal to be a straightforward experiment, this requires one very stable and well-characterized low-frequency antenna and receiver since no spatial information is needed. The total power is $\delta T_{b}$ from Eqn. 1.7 

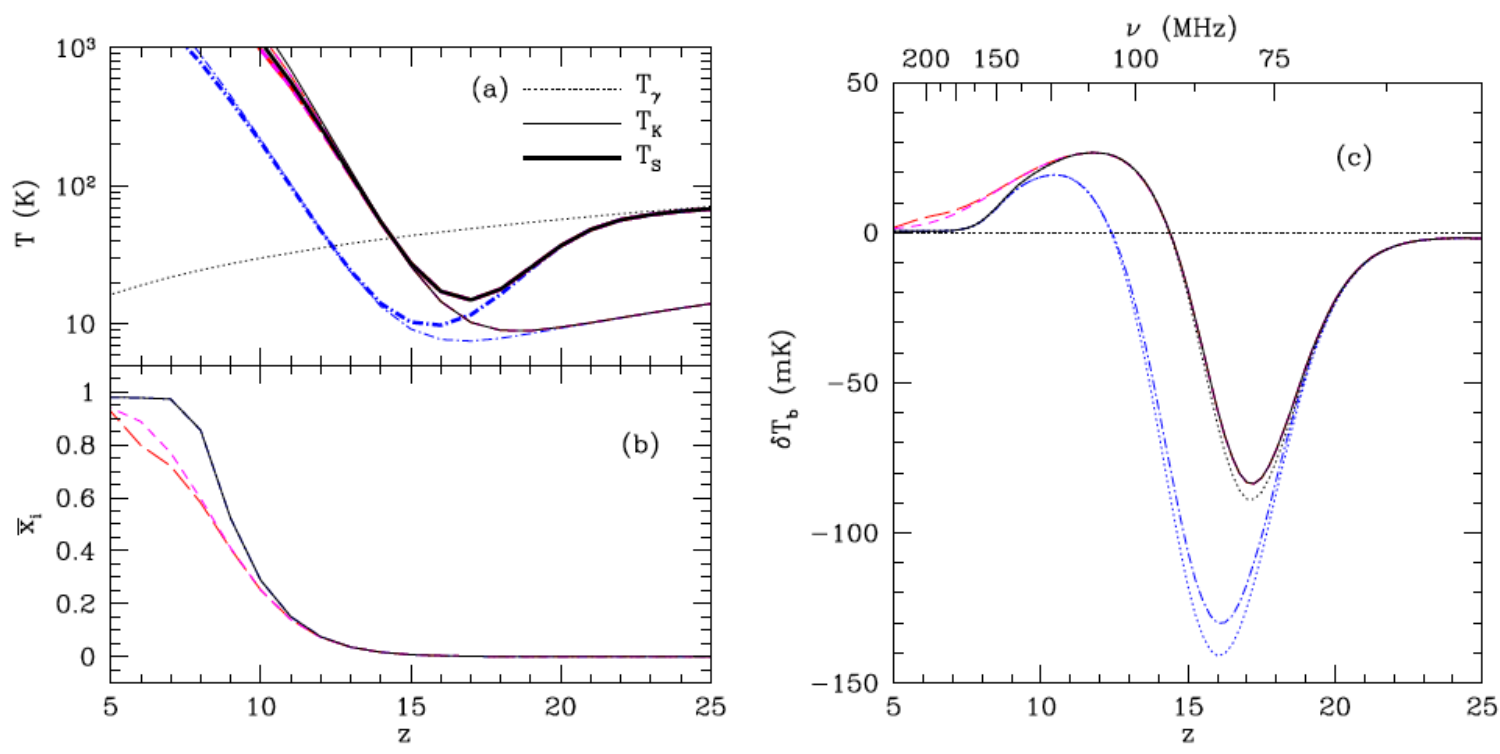

Fig. 1.2.- (a) Temperatures, (b) ionization fraction, and (c) brightness temperature of the IGM with reionization dominated by Pop II stars, from Furlanetto (2006), as described above in Section 1.1.3. The solid black line represents the simple fiducial model. The red and purple dashed curves in all panels allow for feedback to slow reionization. The blue dot-dashed curves in panels (a) and (c) indicate a slower heating of the IGM. The black and blue dotted curves in panel (c) ignore shock heating. 

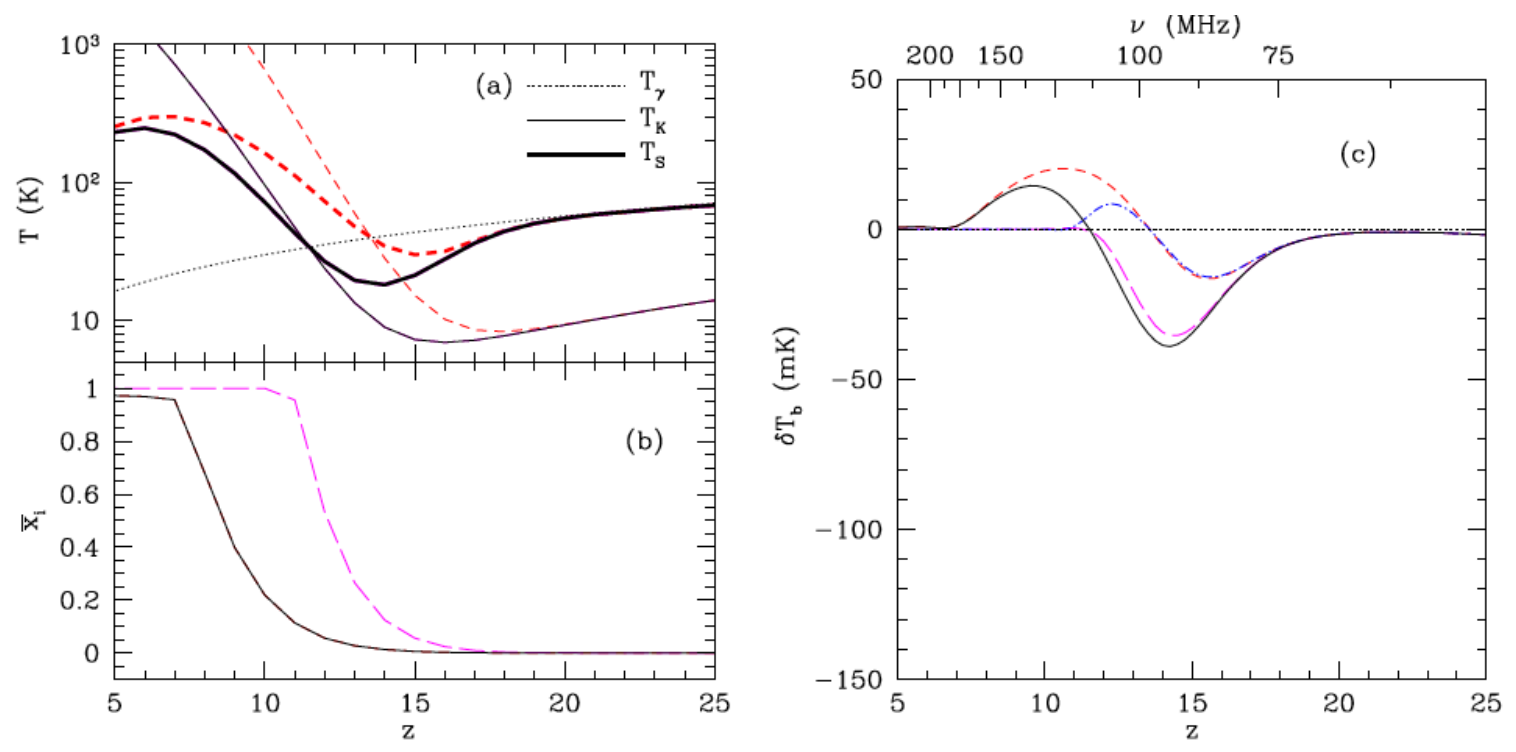

Fig. 1.3.- (a) Temperatures, (b) ionization fraction, and (c) brightness temperature of the IGM with reionization dominated by Pop III stars, from Furlanetto (2006), as described above in Section 1.1.3. The solid black line represents the simple fiducial model. The red and purple dashed curves in all panels allow for feedback to slow reionization. The blue dot-dashed curves in panels (a) and (c) indicate a slower heating of the IGM. The black and blue dotted curves in panel (c) ignore shock heating. 
and evolves over cosmic history depending on how reionization plays out. This evolution is present in the spectrum of the total power. Although PAPER antennas are well-characterized, there is no switch to loads of known noise temperature, so they are not ideal for this experiment.

One such experiment exploring this avenue is EDGES (Experiment to Detect the Global EoR Signature). Their noise limits to date support longer reionization epoch at the limit $\Delta z>0.06$ (Bowman \& Rogers 2010). This rules out the most extremely instantaneous scenarios, but it does not narrow the field of EoR simulations enough to make any further measurements. However, this experiment did provide the very first measurements of the reionization epoch.

\subsubsection{Individual Emission Regions}

The largest individual ionization bubbles near the end of reionization are the most likely targets of experiments that are currently being planned. A simple Strömgren sphere for each galaxy is not sufficient even for a simple model because of galaxy clustering and the large difference between the number densities of the galaxies and of the voids. The so-called "transmission" gap between the $z \sim 6$ quasar Ly $\alpha$ lines and the onset of the Gunn-Peterson trough is thought to be the minimum extent of the HII region of the quasar. However, the pre-existing HII region (before quasar turnon) may have been larger. Furlanetto et al. (2004b) provide an analytic description for the bubble sizes when the ionizing regions follow the halo mass function. $\zeta$ is the number of ionizing photons per baryon, so an HII region is formed when the collapse fraction for the halo is greater than the inverse of the efficiency, or $f_{\text {coll }} \geq \zeta^{-1}$. The Press-Schecter form is used to describe the collapse fraction, or 


$$
f_{\text {coll }}=\operatorname{erfc}\left(\frac{\delta_{c}(z)-\delta_{m}}{\sqrt{2\left[\sigma^{2}\left(m_{m i n}\right)-\sigma^{2}(m)\right]}}\right)
$$

with $\delta_{m}$ now the mean overdensity of the region, $\delta_{c}$ the critical density for collapse, and $\sigma^{2}(m)$ the variance of density fluctuations at a scale $m$. Solving for $\delta_{m}$ and inserting $f_{\text {coll }} \geq \zeta^{-1}$,

$$
\delta_{m} \geq \delta_{c}(z)-\operatorname{erf}^{-1}\left(1-\zeta^{-1}\right) \sqrt{2\left[\sigma^{2}\left(m_{\min }\right)-\sigma^{2}(m)\right]} .
$$

If this is modeled as a linear function in $\sigma^{2}(m)$, using the formalism of Sheth (1998), Furlanetto et al. (2004b) derive the mass power spectrum as

$$
M \frac{d n}{d M}=\sqrt{\frac{2}{\pi}} \frac{\rho}{M}\left|\frac{d \ln \sigma}{d \ln M}\right| \frac{\delta_{c}-\sqrt{2} \operatorname{erf}^{-1}\left(1-\zeta^{-1}\right) \sigma\left(m_{\min }\right)}{\sigma M} \exp \left[-\frac{\delta_{m}^{2}}{2 \sigma^{2}(M)}\right]
$$

where $\rho$ is the average comoving density.

Figure 1.4 shows this bubble size distribution in terms of radius at different redshifts, normalized to the global ionized fraction expected at each redshift. Some of the largest bubbles at the lower redshifts are arcminutes in scale, and will be detectable with interferometers in development. Some of the earliest and most simplistic models show two stages of "bubbles" as recombination occurs after the first stars create small Strömgren spheres, as demonstrated in Figure 1.5.

\subsubsection{Models}

Several models of reionization, whether analytical, numerical, or semi-numerical, have been developed to probe reionization on varying scales and with different levels of complexity, though no one method can include all the necessary complexity. (See 


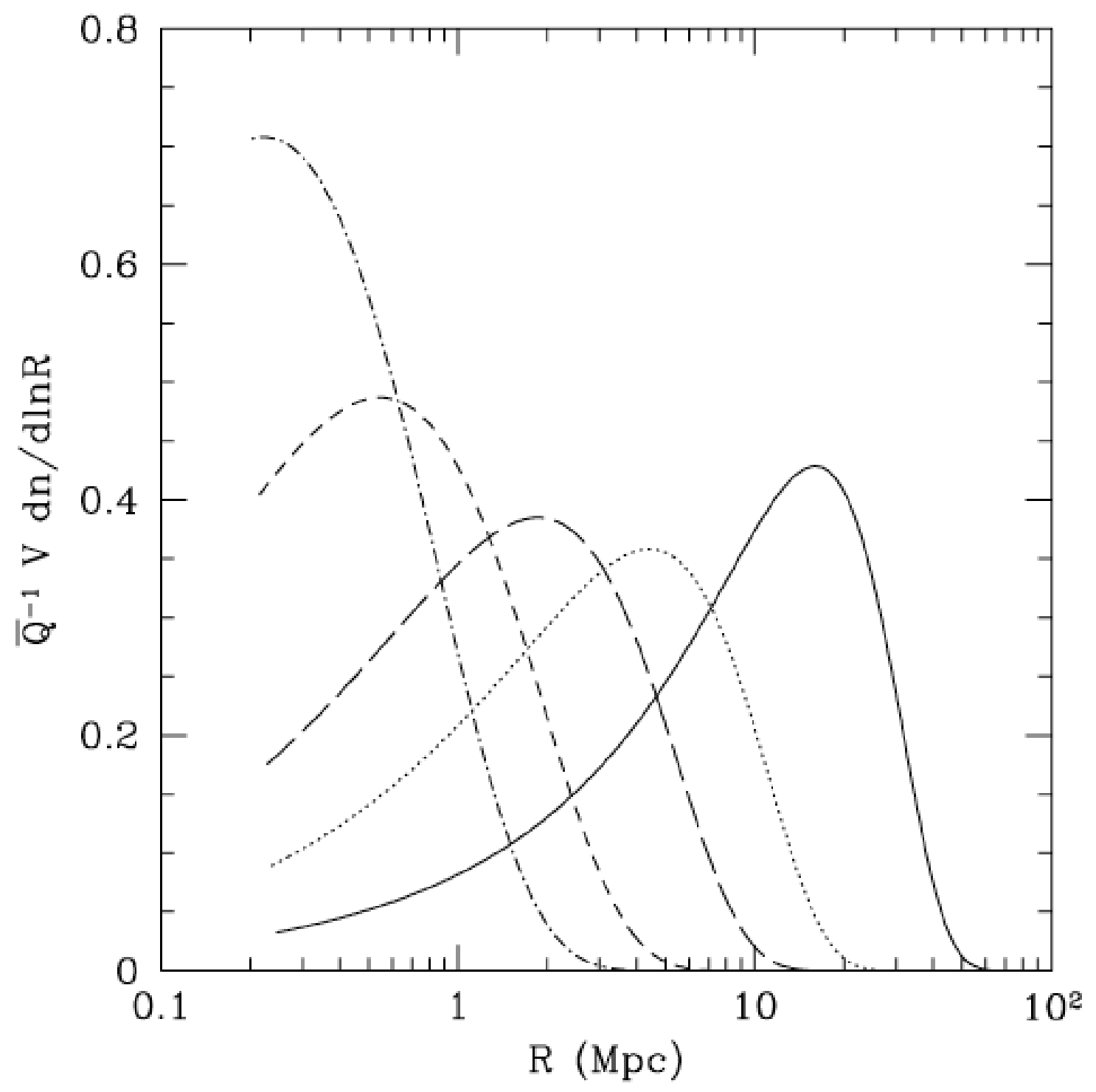

Fig. 1.4. - Bubble size distribution, from Furlanetto, Zaldarriaga, \& Hernquist (2004) and as described by Equation 1.10, where each curve from left to right is for $z=18$, $16,14,13$, and 12 . As is expected, the ionized bubbles generally grow larger at later times. 


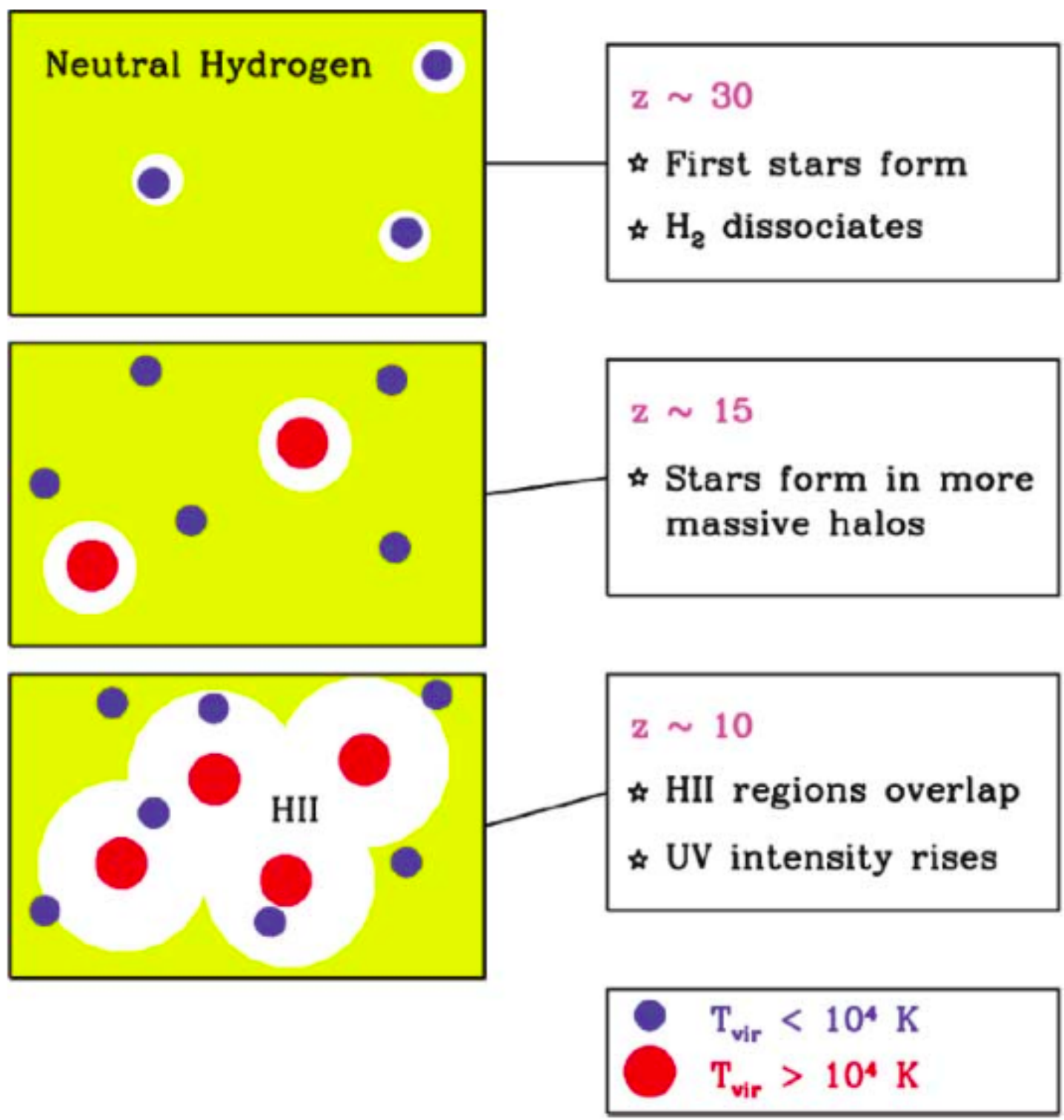

Fig. 1.5. - Cartoon of first and second stage star formation Strömgren spheres, from Barkana and Loeb (2001). The spheres from the very first stage of star formation are thought to disappear due to reionization before the second stage is underway. 
Morales \& Wyithe (2010) for a review.) Many physical processes remain partly or wholly unknown, such as star formation rates, the clumpiness of the intergalactic medium, the contribution of quasars, and the transfer of photons from luminous objects to the intergalactic medium (Mitra et al. 2011). Since reionization is not expected to be instantaneous (Bowman \& Rogers 2010), the evolution of reionization will be available by observing a wide range of frequencies, or redshifts, over which reionization occurs. Thus, characterization of the signal from the EoR will help to define these important missing quantities. The following is the prescription used by Sokasian et al. (2003) to model reionization, with analysis of the predicted signal from this simulation by Furlanetto et al. (2004a).

The simulation describes dark matter as particles and gas as smoothed-particle hydrodynamics in a box of $10 h^{-1}$ co-moving Mpc with $\Lambda$ CDM cosmology. Star formation and feedback in the interstellar medium is modeled such that at high redshifts, star formation is determined by the number density of massive haloes. Simulations of the star formation rate with redshift by Springel \& Hernquist (2003) show that star formation happens primarily in more massive haloes as redshift decreases. The ultraviolet background is turned on suddenly at $z \sim 6$, simulating the end of reionization when uv photons are free to flood the now ionized IGM. It is important to not confuse this with the ultraviolet radiation that is coming from each source and creating HII regions up to and through redshift 6 .

Radiative transfer is calculated after the structure formation above is simulated. That means that reionization in this model will occur without any feedback effects of the radiation on the structure. Some kind of feedback is generally added into models to delay reionization over the entire range as indicated by quasar and WMAP observations. 
The temperature model for the interplay among $T_{S}, T_{K}$, and $T_{C M B}$ is also added analytically. This model assumes from global models that by the time of reionization, $T_{S} \approx T_{K}$ and $T_{S} \gg T_{C M B}$. Finally, a slice of the simulation is "observed" via Equation 1.7, mapping the HI and HII regions determined by the simulation. The result is shown in nine time steps between redshifts 12.1 and 7.6 in Figure 1.6. HII regions form around the luminous sources and grow until the time of percolation, or overlap, after which most of the universe is ionized. The difference between ionized and neutral regions, according to this simulation, is tens of milliKelvin, so imaging these regions may be within the reach of interferometers on the horizon.

The predicted brightness temperature of the HI power spectrum signal is expected to be on the order of $10-20 \mathrm{mK}$, or five orders of magnitude below the population of foreground point sources, as shown in Figure 1.7 (Santos et al. 2005). The sensitivity needed to actually image the HI signal requires something on the order of a Square Kilometer Array (McQuinn et al. 2006).

The first generation of EoR probes, such as PAPER, the Murchison Widefield Array (MWA, Lonsdale et al. 2009) ${ }^{1}$, the Low Frequency Array (LOFAR, Rottgering et al. 2006) ${ }^{2}$, and the Giant Metrewave Radio Telescope (GMRT, Paciga et al. 2011) are gearing towards detecting the power spectrum of the neutral hydrogen signal over a limited range of spatial frequencies within the anticipated epoch. Upcoming, more sensitive instruments such as HERA (Hydrogen Epoch of Reionization Array, Backer et al. 2010) will aim to fully characterize this power spectrum and further define our picture of the early universe.

The brightness temperatures of foreground astronomical sources in this frequency regime can reach several to hundreds of Kelvin and will need to be removed to a

\footnotetext{
${ }^{1}$ http://www.mwatelescope.org/

${ }^{2}$ http://www.lofar.org
} 


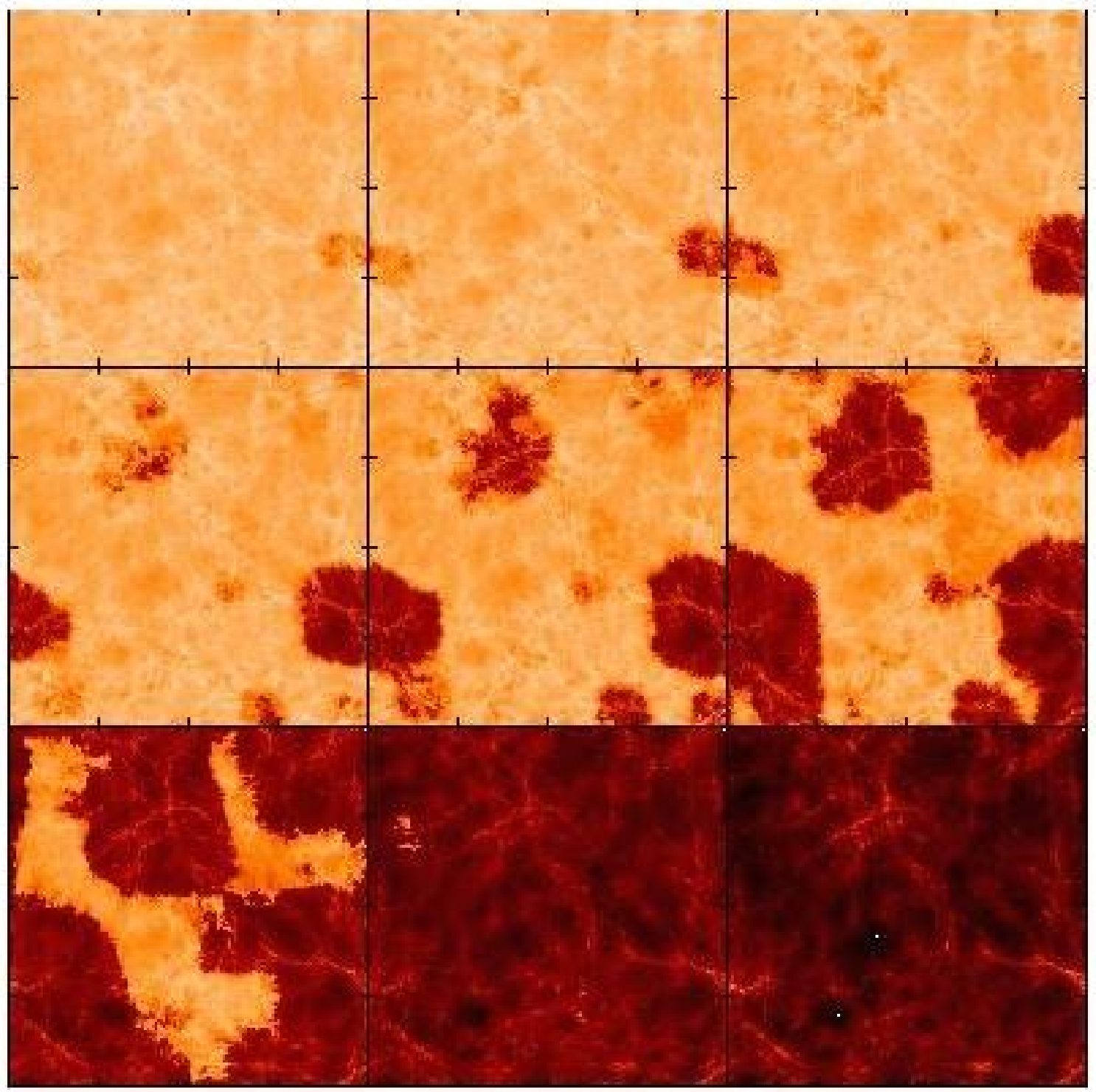

$\log \left(\delta \mathrm{T}_{\mathrm{b}}\right)(\mathrm{mK})$

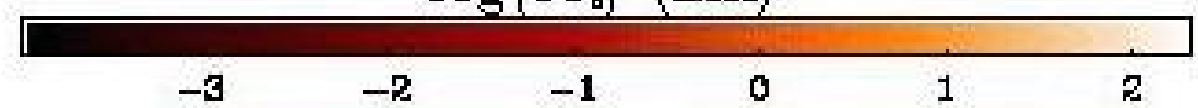

Fig. 1.6.- Simulation of reionization from Furlanetto, Sokasian, \& Hernquist (2004), presenting nine timesteps as a result of the modeling process described above. The color from orange to red indicates the brightness temperature of the HI signal. Evolution proceeds from the first panel where the IGM is nearly completely neutral to the last panel where only filaments of neutral gas are left. 


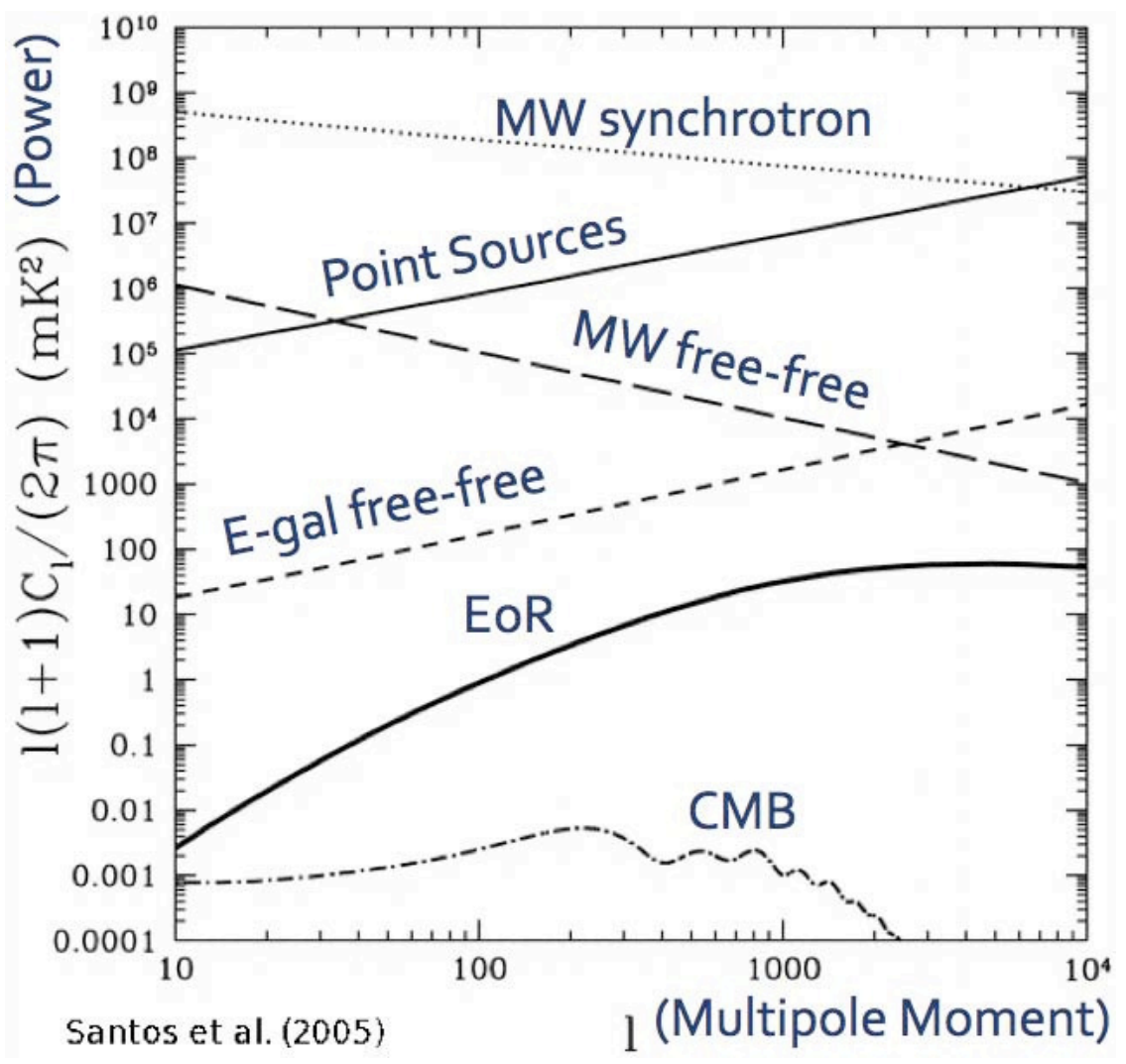

Fig. 1.7. - Milky Way synchrotron emission dominates at all but the smallest size scales (right side of the graph), where the synchrotron emission of extragalactic point sources become important. Shown here are the power spectra of various foreground contributions and of the EoR signal itself. From Santos et al. (2005).

level of tens of mK. Though the expected spectral smoothness of the foreground signals makes this theoretically possible (Santos et al. 2005), implementation of this idea is fraught with challenges. If foreground sources are subtracted imperfectly, residuals will introduce spectral fluctuations which may not necessarily be at the source location, complicating further analysis (Morales \& Wyithe 2010). Irregularities in the ionosphere can cause refractive displacements for bright foreground sources, and imperfect calibration for these displacements will lead to imperfect foreground subtraction. The statistical distribution of these irregularities is not known well 
enough to predict how severe these effects will be, so preliminary observations with EoR-detecting experiments, such as those presented in this paper, are needed to get a handle on the problem.

Several projects are looking to detect the power spectrum of the epoch of reionization fluctuations. This echoes the early work in the 1990s on the CMB, which detected the matter fluctuations in a statistical sense. The EoR power spectrum has one more dimension, however, that of time. The EoR data set will be even richer than the CMB as it probes a span of several hundred million years. This three dimensional power spectrum is represented by wavenumber, $k$, and the power or brightness temperature fluctuation in that wavenumber which corresponds to a particular angular size. The predicted power spectrum of the EoR changes as the inputs to the models differ. Barkana (2009) presents a typical fiducial model of the power spectrum and its evolution in time (or observing frequency) which is reproduced in Figure 1.8. Again, the typical bubble size increases with time, or at higher ionization fractions, though the overall signal decreases in brightness.

\section{$1.2 \quad$ Radio Astronomy Fundamentals}

The power detected by a radiometer can be expressed in terms of a temperature $(T)$ and a voltage gain $(g)$ and is set by the bandwidth $(\Delta \nu)$ of the instrument,

$$
P=g^{2} k_{B} T \Delta \nu
$$

where $k_{B}$ is Boltzmann's constant. The noise power is set by the system temperature, $T_{\text {sys }}$, which is a property of the instrument and includes noise contributions from components in the signal path, as well as from ambient sky. One figure of merit 


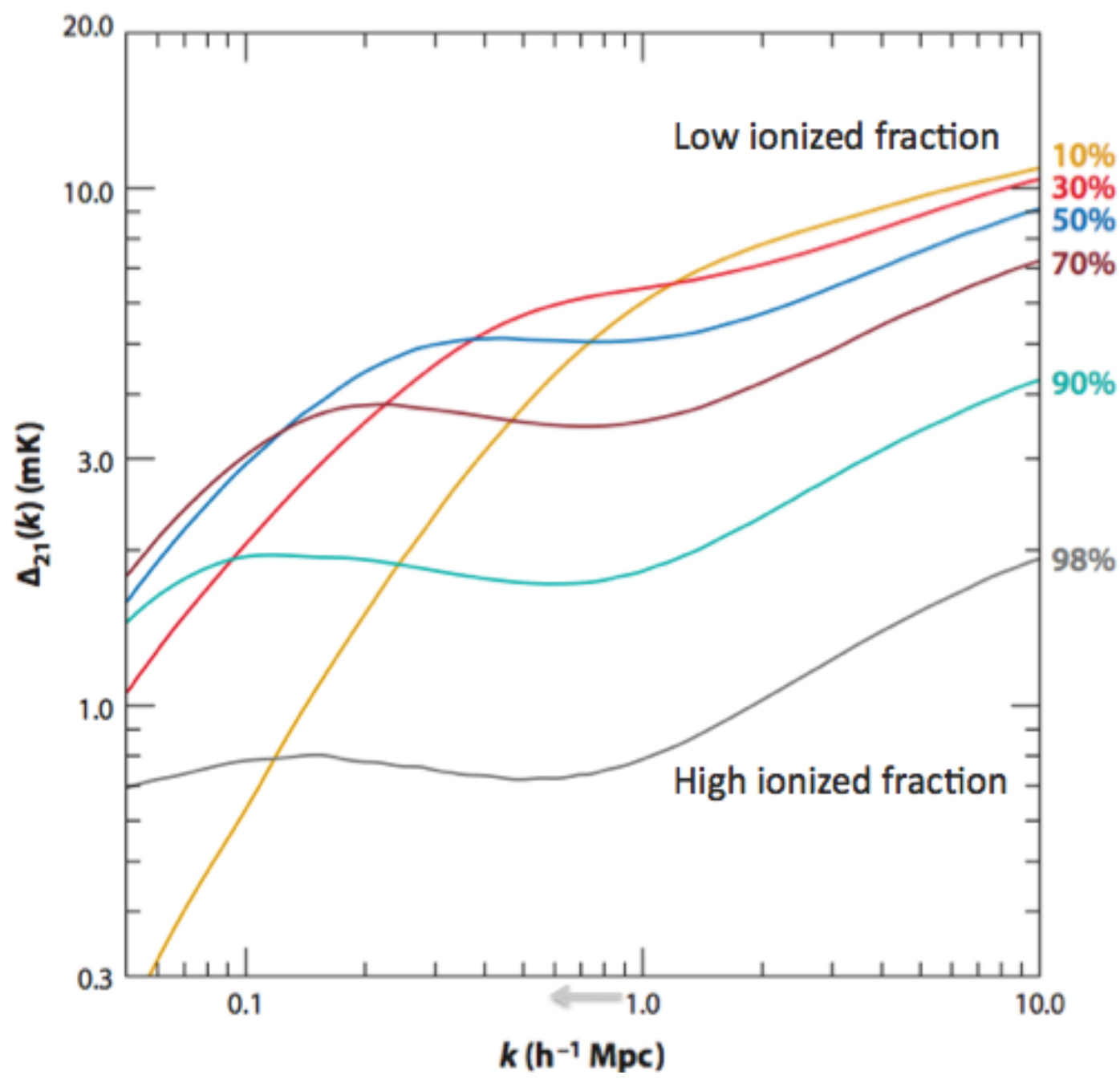

Fig. 1.8. - Evolving power spectrum of the EoR, from Barkana (2009). The colored lines show the power spectrum at different ionization fractions, corresponding to different redshifts. $k$ is the wavenumber and indicates and angular scale, and $\Delta_{21}$ is the brightness temperature of the HI signal.

of a system's performance is the system equivalent flux density $(S E F D)$,

$$
S E F D=\frac{2 k_{B} T_{\text {sys }}}{A_{e}}
$$

where $A_{e}$ is the effective area of the antenna. This is a directionally dependent product of an antenna's isotropic area and the gain in that direction. For a zenith- 
pointing instrument,

$$
A_{e}=G_{0, \max } A_{\text {iso }}=\frac{G_{0, \max } \lambda^{2}}{4 \pi}
$$

with $\lambda$ as the observing wavelength. At this time, it is also convenient to define a sensitivity term,

$$
K=\frac{A_{e}}{2 k_{B}}
$$

In most radio astronomy applications, the brightness temperature of the sky is well below the equivalent electron noise temperature in the receiving electronics. At these frequencies, however, the dominant component of the system temperature is of the Galactic synchrotron, which is several hundred kelvin in our frequency range. The amplifying and receiving components of PAPER were designed to have an electron noise temperature around $100 \mathrm{~K}$. We do not need to cryogenically cool the electronics for PAPER, as is the case in telescopes that work at higher frequencies, since the component from the Galaxy is dominant.

\subsubsection{Interferometry}

The description of the imaging of the sky with a radio interferometer begins with Maxwell's description of an electromagnetic field (Clark 1999). The electric field is treated as a scalar field and it is assumed that the source is at a distance much larger than the scale of the interferometer. Huygens's principle assumes that the field is propagating from the celestial sphere of radius $|\mathbf{R}|$. The correlation of the measured field from two different locations on the ground, $\mathbf{r}_{1}$ and $\mathbf{r}_{2}$, is defined as the visibility function 


$$
V_{\nu}\left(\mathbf{r}_{1}, \mathbf{r}_{2}\right)=\int I_{\nu}(\mathbf{s}) e^{-2 \pi i \nu \mathbf{s} \cdot\left(\mathbf{r}_{1}-\mathbf{r}_{2}\right) / c} d \Omega
$$

where $\mathbf{s}$ is defined as the unit vector $\mathbf{R} /|\mathbf{R}|, I_{\nu}$ is the intensity, and $d \Omega$ is the element of solid angle on the sky. This equation is key to astronomical observations using interferometry. When described in two dimensions, this represents a spatial Fourier transform which can be inverted to produce the intensity distribution on the sky. In reality, however, the measurement of the visibilities is not perfect, so calibration is needed to correct for baseline lengths, delay through the atmosphere, quantization, bandpass shapes, etc. Calibration and imaging techniques have been developed over the past few decades that have worked especially well at centimeter and millimeter wavelengths and over small fields of view. Low frequencies and widefields, such as is required for EoR experiments, are more difficult to handle because of a variable ionosphere and wide-field imaging effects that make the two dimensional approximation invalid.

Interferometry is the method by which signals from multiple antennas are crosscorrelated to make an image of the sky. When two antenna signals, $s_{i}$ and $s_{j}$, are combined in such a way, the power measured by the interferometer is,

$$
\left\langle P_{i j}\right\rangle \propto\left\langle\left(s_{i}+n_{i}\right)\left(s_{j}+n_{j}\right)\right\rangle=\left\langle s_{i} s_{j}\right\rangle
$$

and the noise from each, $n_{i}$ and $n_{j}$, is assumed to be uncorrelated with the others ("Sensitivity" from "Synthesis Imaging in Radio Astronomy," Wrobel \& Walker 1999). The resulting power includes the gains and sensitivities of each antenna along with an interferometer efficiency factor, $\eta_{s}$, 


$$
\left\langle P_{i j}\right\rangle=\frac{g_{i} g_{j}}{\eta_{s}} \sqrt{K_{i} K_{j}} k_{b} \Delta \nu S
$$

with $\mathrm{S}$ as the flux density. The noise produced by correlation is derived by assuming Gaussian statistics and taking the expectation value of the power minus the square of the mean. The derivation can be followed in full in Wrobel \& Walker (1999) to produce the minimum detectable signal by two identical elements when the source temperature is less than the system temperature and $t_{\text {avg }}$ is the averaging time of the correlator,

$$
\Delta S_{i j}=\frac{S E F D}{\eta_{s} \sqrt{2 \Delta \nu t_{a v g}}},
$$

where $S E F D$ is as described in Equation 1.12.

This is then translated into noise in the synthesis image. Each point in the image plane includes information from the visibility, or spatial frequency, plane sampled by the interferometer. Assuming no weighting or tapering of the visibility plane, the noise in the central pixel is the noise power calculated above divided by the square root of the number of samples, or more simply, the number of baselines in the array, $N(N-1)$, times the number of samples taken with time $\left(t_{\text {int }} / t_{\text {avg }}\right)$,

$$
\Delta I=\frac{S E F D}{\eta_{s} \sqrt{2 N(N-1) \Delta \nu t_{i n t}}} .
$$

PAPER will consist of an array of dipole antennas, each on one groundscreen to increase gain. The maximum gain at zenith of this system is $8.7 \mathrm{dBi}$. The effective area of one of these is then $2.33 \mathrm{~m}^{2}$ (according to equation 1.13). The system temperature is expected to be $100 \mathrm{~K}$ or less. Therefore, the $S E F D$ of one antenna is $118 \mathrm{kJy}$. The large number must not be misleading, for it is partially dependent on a 
very small antenna size. For example, the imaging sensitivity for an 8-hour run with a 16-element array is $4.3 \mathrm{mJy}$, assuming that the bandwidth chosen to ignore RFI is $70 \mathrm{MHz}$ and the efficiency factor is $\sim 0.88$ for a 4-quantization level correlator. This is, however, under simplistic assumptions that the effective aperture and image noise calculations are the same over the whole sky. Also the effective area of an array of dipoles may not be strictly described by the scaling of an individual element by the number of dipoles, especially when elements are in each other's near-field.

This can be used to see how long it would take to get imaging sensitivity to the level of the signal of EoR. The signal decrement in the HI signal from an ionized bubble at high redshift is expected to be on the order of $10 \mathrm{mK}$. The flux density from decrement is given by the Rayleigh-Jeans approximation of Planck's law,

$$
S_{\nu}=\frac{2 k_{B} T \theta^{2}}{\lambda^{2}}
$$

where a bubble is $\sim 10$ arcminutes in size and therefore 0.06 mJy in flux. With a bandwidth of $100 \mathrm{MHz}$, feasible in the radio frequency quiet area of South Africa, and 128 elements with the same effective aperture, efficiency factor, and system temperature as above, an integration time of 430 hours is needed to reach this sensitivity. If a piece of sky was within good observing limits (i.e. plane of the Milky Way close to the horizon, stable ionosphere) for 12 hours per day, this amounts to 36 days. However, to achieve a $3 \sigma$ detection, these integration times would be be multiplied by 9 . That is, the best case scenario would involve 323 days of integration. An array with twice as many elements could achieve the $3 \sigma$ limit in 80 consecutive days if integrating on the South Celestial Pole. Clearly, number of elements has a great effect on the sensitivity since $\Delta I \propto[N(N-1)]^{-1 / 2} \simeq N^{-1}$ but integration time only affects the sensitivity as $\Delta I \propto t^{-1 / 2}$. However, $10 \mathrm{mK}$ may be a pessimistic estimate of the difference in signal 
between the HII and HI regions, so for $20 \mathrm{mK}$, the 128-elements array can reach $3 \sigma$ sensitivity in 81 days with 12 hours of observing per day. Of course, this assumes an otherwise perfect instrument, perfect calibration, and ignores the fact that PAPER is a drift-scan instrument, meaning that a patch of sky will pass through the primary beam over the course of a day, only spending a small amount of time in the most sensitive part of the beam.

\subsection{PAPER-specific Challenges}

The EoR experiment, and low-frequency radio astronomy in general, has unique challenges. Traditionally, the system temperature is dominated by the thermal noise of the radiometer itself, and the sky contributes only a small fraction. Thus, many receivers, such as those on the VLA, are cryogenically cooled to reduce this noise temperature. Due to the steep spectrum of synchrotron emission, however, the emission from the Milky Way (MW) and from extragalactic sources is much brighter at low frequencies. (It is for this reason that Grote Reber did not detect anything with his first system in the GHz-regime and only detected the MW synchrotron after dropping down to $160 \mathrm{MHz}$.

This brighter sky temperature is a help to low-frequency telescopes in that the sky noise dominates, so there is no need to cryogenically cool amplifiers and receivers. Having ambient temperature amplifiers saves on the cost of building the instrument. However, this sky temperature is a hindrance to the EoR experiment since the hydrogen signal is so much fainter than the foreground objects. Thus the focus is on precision calibration. In addition, the Milky Way synchrotron is partially polarized, though most of this emission is on large scales and is thus "resolved out" by the interferometer. 
Not only will PAPER need to be sensitive enough for the EoR experiment, but the system must be stable over the long periods of time necessary to achieve the desired sensitivity. Since the components are not cryogenically cooled, one of the main issues turns out to be the dependence of the gain in various amplifiers in the signal path on the ambient temperature. The system is laid out in Chapter 2, and this stability issue is further explained in Chapter 3.

Low-frequency observatories also have to deal with the scourge of the ionosphere. Though optical astronomers typically deal with scintillation and isoplanatic patches, the atmosphere does not tend to affect radio waves at centimeter wavelengths. Below a few hundred $\mathrm{MHz}$, however, the ionosphere becomes a significant refractive layer. Turbulence in Earth's ionosphere made observations difficult in this regime for several decades. The ionosphere is explored further in Chapter 4. The lessons learned from all of these experiments and the impact on future astronomical measurements with PAPER and other low frequency telescopes is summarized in Chapter 5.

My specific contributions to the PAPER project include a major role building and deploying the Green Bank prototype arrays from 2006 to 2011 and one South Africa deployment in 2011. These stages are summarized in Table 1.1. Over the course of this work, I have learned to design array configurations, use progressively better surveying equipment to mark antenna positions in the field, assemble and orient groundscreens, build amplifiers and receivers, troubleshoot the correlator, cut, connectorize, and roll out RG-6 cable, test and troubleshoot various components of the RF system, and take care of various other tasks in order to bring the array to full operation. I calibrated the antenna positions and delays for progressively larger data sets and explored imaging with wide bandwidths and large fields of view inherent to our type of instrument. I performed the system temperature calibration experiment detailed 
in Chapter 3 and helped engineering graduate student Chaitali Parashare become familiar with my fitting routines when she undertook the project. I calibrated and modeled the positions of bright sources in the sky as explained in Chapter 4 and drew the conclusions about the effects of the ionosphere on PAPER calibration presented in this work. These early experiments are important first steps towards the goals of detecting the signal of the Epoch of Reionization and of doing science with the low frequency arrays currently under construction.

Table 1.1: PAPER Deployments

\begin{tabular}{lcc}
\hline Deployment Dates & Location & $N_{\text {ants }}$ \\
\hline 2004 & Green Bank & 4 \\
$2006-2008$ & Green Bank & 8 \\
2007 & Western Australia & 4 \\
$2008-2009$ & Green Bank & 16 \\
$2009-2012$ & Green Bank & 32 \\
2009 & South Africa & 16 \\
2010 & South Africa & 32 \\
$2011-2012$ & South Africa & 64 \\
\hline
\end{tabular}




\section{Chapter 2}

\section{PAPER Instrument}

\subsection{Hardware}

Most radio telescopes have similar components, despite their diversity in size and structure. This is no different with the PAPER system. At the front end, an antenna responds to the electromagnetic radiation in the chosen frequencies. In the simplest case of a dipole, electrons are excited by the radiation to move within the wire, creating a current (Condon \& Ransom 2007).

In the case of PAPER, it is required to have an antenna with a smooth reception pattern, or beam, over a rather wide range of frequencies. In order to capture the entire reionization history as predicted by high-redshift quasar observations and cosmic microwave background (CMB) polarization (Fan et al. 2006; Mortonson \& Hu 2008), a sleeved dipole was designed for a smooth pattern from 110-180 $\mathrm{MHz}(z \sim 7-12)$ (Parsons et al. 2010). (See Figure 2.1.)

At each of the two antenna terminals is a pair of low-noise amplifiers followed by a 180 degree hybrid power combiner to form an active balun with about $30 \mathrm{~dB}$ of power gain. The total noise in the system is mostly determined by that first element, so a 


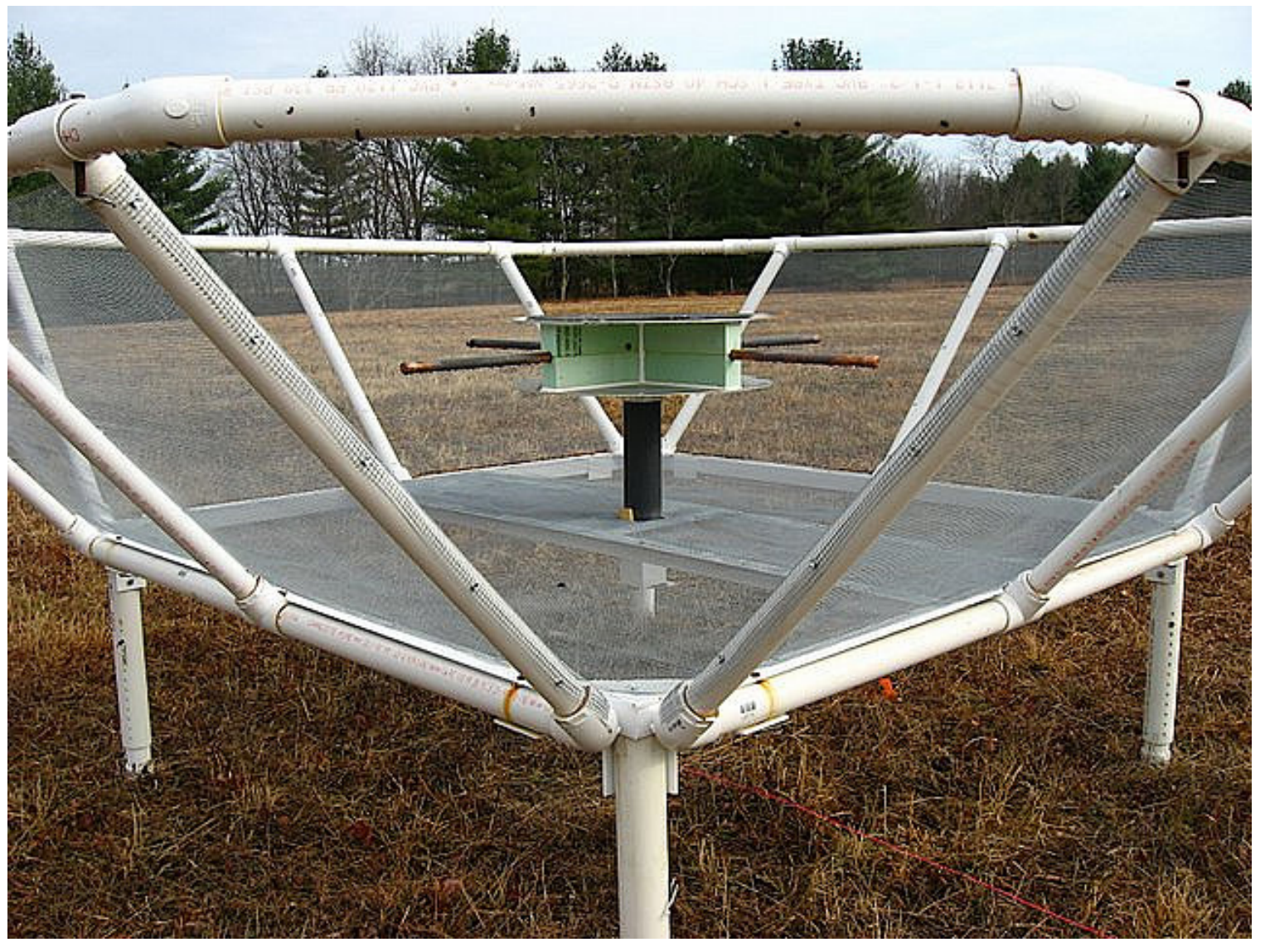

Fig. 2.1. - Single PAPER element or antenna. The copper dipoles in this structure are held in place by dialectric foam and epoxy in between two precisely spaced aluminum plates. The more recent design replaces the dielectric foam with plastic spacers and is thus easier to assemble. The antenna assembly is mounted on a grey PVC pipe containing the first stage of amplification and sits on a steel and PVC groundscreen. 
low-noise amplifier is necessary. This allows the signal to then pass through $150 \mathrm{~m}$ of coaxial cable. The RG-6, 75-ohm cable feeds into a temperature-controlled hut which houses the receivers for each antenna in the field. The receivers filter and further amplify the signals which are then sent to a digitizer and correlator for processing.

PAPER is an experiment geared specifically towards detecting the power spectrum of the EoR. It is a collaboration among the University of Virginia, the University of California at Berkeley, the National Radio Astronomy Observatory, the University of Pennsylvania, and the Square Kilometer Array South Africa. The details of the first 8-antenna deployment in Green Bank, West Virginia, can be found at Parsons et al. (2010). The block diagram is shown in Figure 2.2.

Various PAPER deployments have occurred in the National Radio Quiet Zone $(\mathrm{NRQZ})^{1}$ in Green Bank, West Virginia, operated by the National Radio Astronomy Observatory $^{2}$. This site is still used for engineering and evaluating new PAPER hardware designs, correlators, and antenna configurations, as it is a short drive from Charlottesville, VA. This site currently has 32-antennas on a plot of land at the edge of the Observatory site.

However, there is still significant interference for the sensitive EoR experiment which is the end goal of this project, and the NRQZ is only policed down to $300 \mathrm{MHz}$. Therefore, the full 128-antenna experiment will take place in a designated radio quiet zone in the Northern Cape of South Africa. A 64-antenna array is currently deployed near the KAT-7 demonstrator array in the Karoo ${ }^{3}$.

The PAPER antenna is a transit-telescope that sees a large part of the sky at any given time with a beam FWHM of $\sim 45^{\circ}$ (see Figure 2.3). The dual-polarization sleeved dipole design includes copper crossed dipoles between two aluminum disks

\footnotetext{
${ }^{1}$ http://www.gb.nrao.edu/nrqz/

${ }^{2}$ https://www.nrao.edu/

${ }^{3}$ http://www.ska.ac.za/meerkat/kat7.php
} 


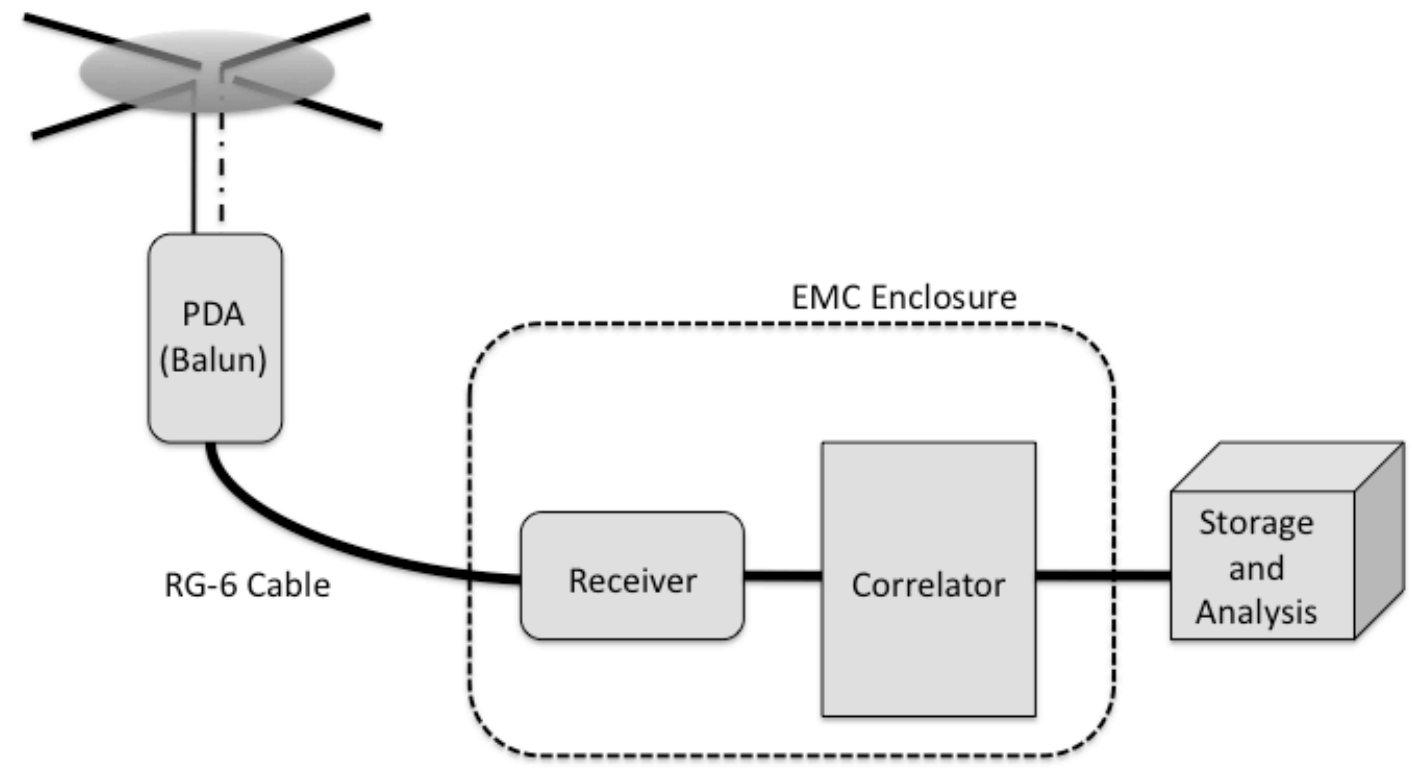

Fig. 2.2.- Block diagram of individual PAPER element, as described in Section 1.3. The cosmic radiation collected at the dipole is first amplified in the balun then sent along 500-ft of RG-6 cable. The signal then enters a shielded hut or shielded boxes within a hut and is further amplified and filtered by the receivers. The signal is then digitized and correlated and sent offsite for further analysis. 
that help ensure this wide frequency coverage. The metal parts are kept separate by plastic spacers, and the entire dipole can be assembled in just a few minutes. This sleeved dipole is centered on a wire-mesh groundscreen with "flaps" extending from each edge of the square at a $45^{\circ}$ angle to improve forward gain. This trough-reflector also serves to eliminate gain variations from changing climate conditions affecting Earth ground, as well as potentially bright sources of interference near the horizon.

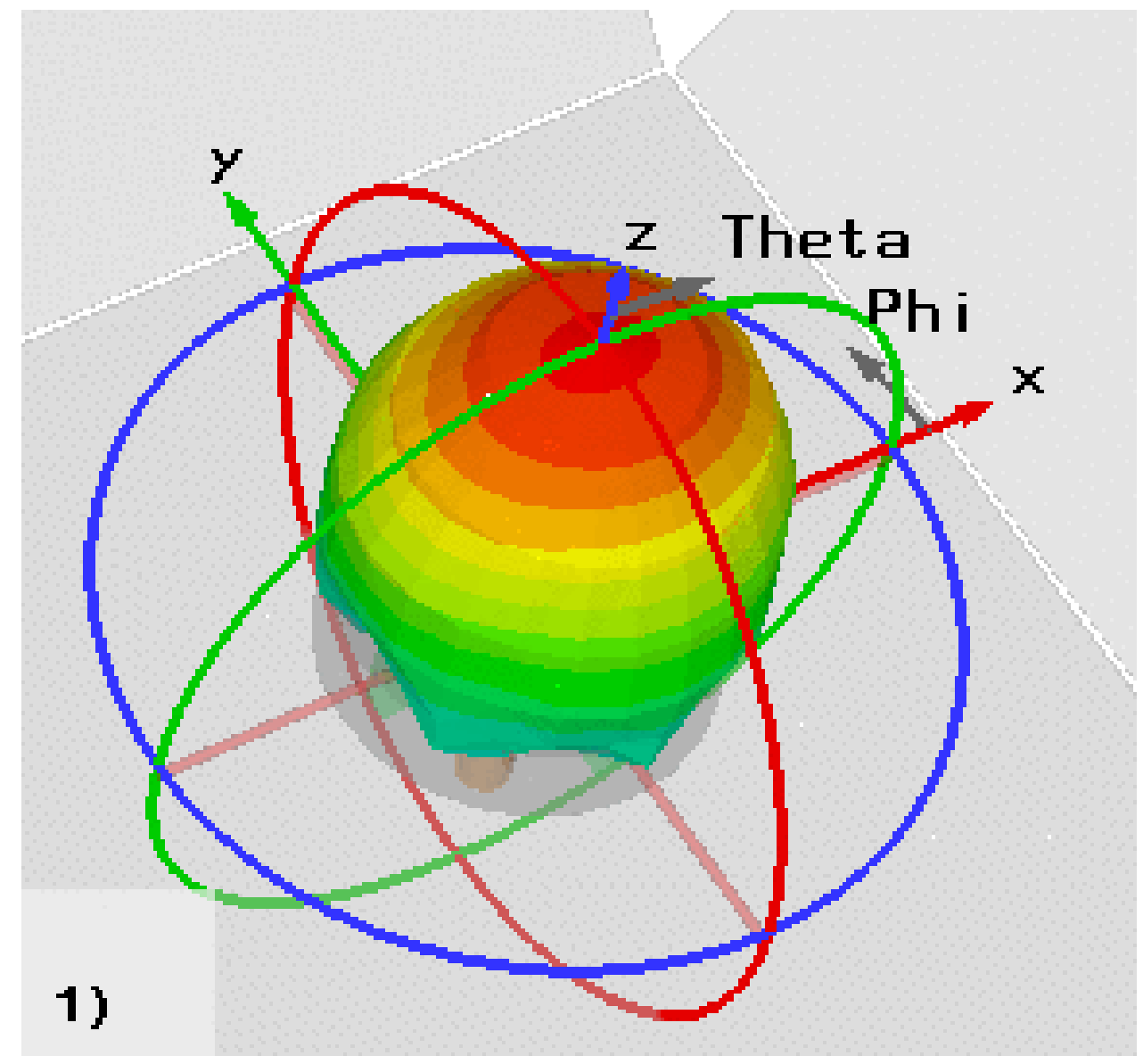

Fig. 2.3.- Visualization of dipole beam pattern from Computer Simulation Technology's Microwave Studio. The beam is slightly elliptical for a single dipole. The peak gain is $12.4 \mathrm{dBi}$.

From this point, the signal travels to a low-noise pseudo-differential amplifier (PDA) housed in the supporting mast for the dipole. This PDA has been designed 
for smooth frequency response to minimize any errors that a complicated spectral shape would have on calibration. Each amplifier has three cascaded gain stages based on NEC NE46100 bipolar junction transistors. The gain through this stage is $\sim 32 \mathrm{~dB}$, however, it can depend on the ambient temperature on the balun housing by $-0.025 \mathrm{~dB} \mathrm{~K}^{-1}$ (Parashare 2011). Thus the actual gain at a given time is

$$
G_{\nu}(t)=G_{\nu}\left(t_{0}\right) H\left(T(t)-T\left(t_{0}\right)\right)
$$

where $G_{\nu}(t)$ is the total gain as a function of time, $G_{\nu}\left(t_{0}\right)$ is the temperatureinvariant gain, $H$ is the temperature-variant gain, and $T(t)$ and $T\left(t_{0}\right)$ are the timevariant temperature and reference temperature, respectively.

One approach to reducing the change in temperature is to paint the top of the aluminum disk on each antenna with the same white paint that is used for the Green Bank Telescope to minimize infrared absorption. In addition, much effort has been made to quantify and correct for the temperature-dependent gains along the entire analog system (Parashare 2011).

The signals are then transmitted to a central location via rugged coaxial cables. These cables are not buried and must endure sun exposure, precipitation, and the occasional gnaw by a local critter, while keeping the signal spectrally smooth. PAPER uses RG-6, 75-ohm cable with a polyethylene jacket that fits our specifications at a low cost. Signal attenuation over a $150 \mathrm{~m}$ cable run is approximately $12 \mathrm{~dB}$ at $150 \mathrm{MHz}$, with a slope of $+0.034 \mathrm{~dB} \mathrm{MHz}^{-1}$. The cable transmits signal for each polarization and for power to the pseudo-differential amplifier.

The receivers follow with more amplification stages $(\sim 60 \mathrm{~dB})$ and a band-limiting filter to restrict the signal to the ranges 130-185 MHz in Green Bank and 120-205 MHz in South Africa. The range is more restricted in Green Bank to mitigate the effects 
of radio-frequency interference (RFI) at the low and high ends of the band from air traffic control and digital television. These receivers prepare the signal for the digital correlator. The amplification stages are also subject to a temperature-dependent gain with a coefficient of $0.045 \mathrm{~dB} \mathrm{~K}^{-1}$. Each dual-polarization receiver is housed in a specially designed EMC enclosure which mitigates crosstalk between receivers and prevent self-interference.

Aperture synthesis is performed by a real-time digital FX correlator based on Field-Programmable Gate Array (FPGA) processors (Parsons et al. 2008). These correlators are based on a flexible architecture whereby signal processing engines transmit packetized data through commercially available 10 gigabit ethernet switches that are responsible for routing data between boards. This architecture, along with analog-to-digital converters, modular FPGA-based signal processing engines, and a software environment for programming, debugging, and running them, were developed in collaboration with the Center for Astronomy Signal Processing and Electronics Research (CASPER) at the University of California, Berkeley.

The correlator is also protected with RFI shielding with a copper-screen box with a wooden frame (Bradley 2006). This alleviates self-interference and allows us to use our equipment in radio-quiet zones shared with other research telescopes. In Green Bank, the receiver boxes and correlator cage are housed in a small hut with a passive system of temperature control using an HVAC system.

\subsection{Deployment and Configurations}

PAPER has been built in a series of larger and larger deployments (see Table 1.1) with a focus towards testing and vetting every stage of the signal path and data pipeline. After the initial design for the PAPER antenna was tested in 2005, a small 
four-element array was deployed in Green Bank along an east-west baseline with a prototype correlator. This first stage involved regulating input power levels to the correlator so as to not overload the analog-to-digital converters and introduced the type of landscape management that would affect future deployments.

Following this, PAPER got a more permanent site in Green Bank in what Don Backer dubbed "Galford Meadow" after the farmer who owned the plot of land before the observatory was built. No longer under the obstructing gaze of the 85-foot telescope or in the path of the site mowers, PAPER was free to expand both in size and in scope. The first eight antennas on the site were placed on a circle 455 feet in radius centered on the small electronics hut that was built for power and internet connectivity. The array design was chosen for ease of surveying since locations were marked out with a 1901 theodolite to measure angles, a 300-foot tape measure, and a hand-held compass. The antennas were spaced around this imaginary circle such as to give the best $u v$-coverage that can be obtained under such tight constraints. That is, the antennas are spaced as to sample of much of the Fourier space as possible to obtain maximum imaging sensitivity.

Several other challenges presented themselves as the array began to develop further. The RG-6 cable that was inexpensive enough to buy in 1000-foot reels was also a target for the local fauna. Occasionally, the cable had to be searched, repaired, or even replaced in cases where the rubber insulation or underlying wire had been chewed through. A plastic cable conduit was not a suitable solution as it could also be penetrated by the inhabitants of the meadow, and it was difficult to feed 500 feet of cable through such a conduit in the first place. It was necessary, in addition, to put restrictions on mowing Galford Meadow to a certain time of year when the cable could be removed temporarily such that it didn't get chewed up by the tractor blades. 
Initial experiments with the eight-element array focused on simple tasks that would be necessary for the larger project to succeed. One such experiment is detailed in Chapter 3, where the total power from a single element was measured and tracked throughout the day in an effort to measure the system temperature in the field. This is in line with PAPER's philosophy in which every aspect of the instrument that can be known beforehand is carefully measured to insure accurate calibration.

Another experiment was to measure the primary beam pattern of the antennas using passing satellites from the OrbComm system. This constellation of satellites operates at $137 \mathrm{MHz}$ and crosses the beam in many locations. The satellite signals are extremely bright and can be detected in a small fraction of time. Rich Bradley developed the concept and built the first prototype instrument for measuring the beam pattern using OrbComm satellites. Paul Ries refined and extended the measurements in Green Bank. Jonnie Pober performed a detailed measurement of the beam pattern assuming beam symmetry.

First attempts at imaging were crude, at best. As this project was in collaboration with the University of California Berkeley, the data were first written to a uvfile format read by the astronomy software package Miriad. Getting back to the very basics of interferometry, the first step of any array is to properly "phase," or point, to the bright sources in the sky. Since PAPER is a transit telescope, observations could be "pointed" only electronically after the fact by adding a phase term to the visibilities. In 2009, a four-antenna deployment was carried out in Western Australia by Don Backer and Erin Mastrantonio to experiment with temperature stability and a much improved RFI environment.

The results of the 8-element array in Green Bank were reported in Parsons et al. (2010). An initial sky model could be used to achieve a first level of calibration from 
which a more accurate sky model could then be constructed. Full-sky imaging was achieved to a level of $10 \mathrm{mJy}_{\text {beam }}{ }^{-1}$ in the quietest parts of the sky, though significant sidelobes were still present around the brightest sources. The power spectrum of the noise reached $5.8 \mathrm{~K}$, still three orders of magnitude above the predicted EoR power spectrum, and was dominated by point sources.

The antenna was redesigned to improve forward gain and thus the sensitivity of the array. The groundscreen added four "flaps" at 45-degree angle from the bottom ground screen. Also, the Green Bank shop made the frame out of steel base and PVC pipes rather than wood, which would be more robust in the field. The primary beam was reduced in width slightly, but there was a gain improvement at the zenith from $8.90 \mathrm{dBi}$ to $12.4 \mathrm{dBi}$. However, the new design made the antennas less portable in the field, so care was taken to get the positions correct.

The new antenna design was deployed in Green Bank with a 16-element array in 2009, at which point the antennas were finally large enough to be spotted on Google Maps (see Figure 2.4). Construction continued with 16 elements on a circle around the central hut, once again spread out such that maximal $u v$-coverage was achieved. The improved method of choosing positions involved borrowed surveying equipment from GB staff, including a laser theodolite to measure angle and distance.

The array was expanded to 32 elements as independent contractors were brought in to build the components of the antenna and groundscreen. The new method of determining antenna positions used a GPS receiver during a brief window when the GBT and other radio telescopes were down for maintenance. This improved the accuracy such that calibration was much easier down the line (see Section 2.3). This also removed the restriction of the antennas being on a circle around the hut.

In 2009, PAPER components were built and assembled at the SKA candidate site 


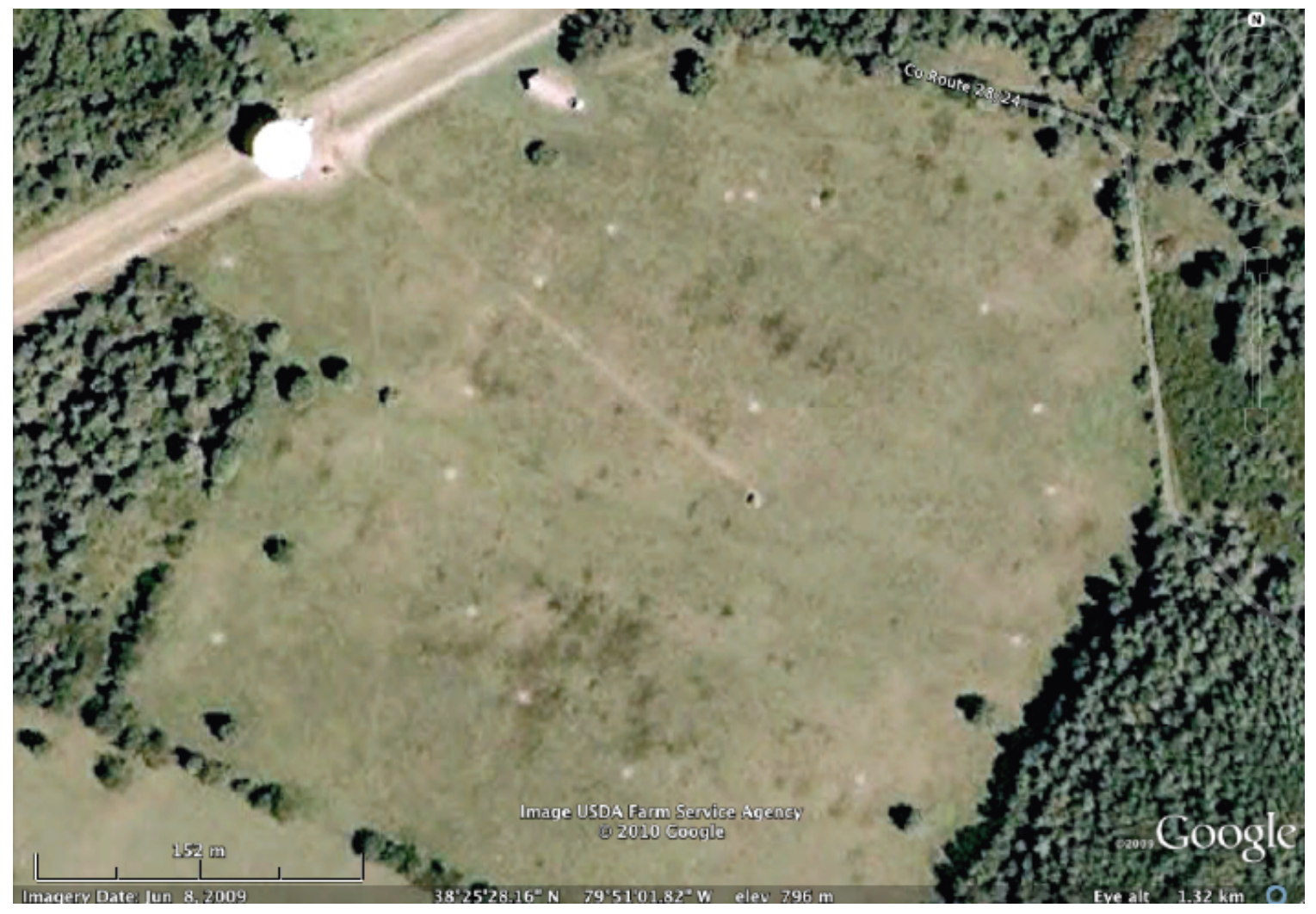

Fig. 2.4. - Aerial view of the 16-antenna array in Green Bank from Google Maps, centered on the Galford Meadow. The road, central hut, and 16 groundscreens are visible. The outermost antenna in the Green Bank Three-Element Interferometer can be seen at the top left as a white circle. 
in the Karoo. This is one of two candidate sites for the Square Kilometer Array, a large international collaboration with the goal of building a wide-band radio array with a collecting area of a square kilometer. The science advisory board recently recommended the South Africa site over the site in Western Australia, but the array will be split between both sites.

In June 2011, there were already 32 elements on site. 32 more groundscreens and antennas, plus some spares, were sent to one part of the PAPER group, who assembled them on site. By this time, the dipole had evolved to one with electrically the same properties, but made with plastic spacers rather than the foam and epoxy that were shown to deteriorate in exposed weather conditions. The new baluns and receivers for these 32 antennas came from Charlottesville with the front-end deployment team. The next week was set aside for inspecting every antenna and groundscreen on site for obvious signs of wear and tear and to repair any major breaks in the groundscreens or bent feeds in the dipoles. RG-6 cable was rolled out to the new antennas, with the help of two interns from Pretoria.

The baluns were installed in the new dipoles and the old ones inspected for signs of corrosion or broken connections. Finally, the groundscreens were revisited to make sure that each was level and aligned with the compass directions. Several were found to be out of alignment, but could be fixed easily by adjusting the height of the telescoping legs. Getting the alignment and levels correct is essential to using the primary beam information for calibration so that the maximum gain is directly at zenith and the slight ellipticity of the beam can be taken into account properly. Several other members of the team worked to build the racks inside the RFI-shielded container, install the receiver boxes, and get the new correlator code compiled and working. 
Once the array was constructed, all of the signal paths were tested and checked for dead channels or obvious reflections, and baluns and receivers were replaced as necessary. To minimize RFI from on-site activities, observing was restricted to nighttime hours. Since power and internet cables had not yet been run out to the PAPER container, the diesel generator was filled every evening before leaving the site and the data disk retrieved each morning. Although it was winter at the site, the container's air conditioning unit had to run almost constantly to compensate for the heat generated by the correlator. Unfortunately, the unit failed several times after the team left the site, requiring maintenance by the SKA staff to alleviate the problem.

\subsection{Calibration and Imaging}

Initial PAPER experiments wrote data files in Miriad uvfile format, however, no existing interferometry package had all of the tools for working with PAPER's unique dataset. AIPS has some capability for dealing with the 74-MHz system on the VLA which has a field of view of several degrees, but PAPER has a primary beam of several tens of degrees. Though $\mathrm{CASA}^{4}$ (Common Astronomy Software Applications) was being designed with a wider range of features to encompass the broadband EVLA and ALMA, development was still in early stages at the beginning of the PAPER project. A new software package was written in Python by Aaron Parsons of UCB. Using much of the C-powered functionality of numpy and scipy, Astronomical Interferometry in PYthon, or aipy ${ }^{5}$ was created and expanded along with the PAPER array deployments, eventually becoming parallelized for larger and larger datasets.

Initial calibration steps included matching up data with correct frequencies, time,

\footnotetext{
${ }^{4}$ http://casa.nrao.edu/

${ }^{5}$ http://pypi.python.org/pypi/aipy
} 


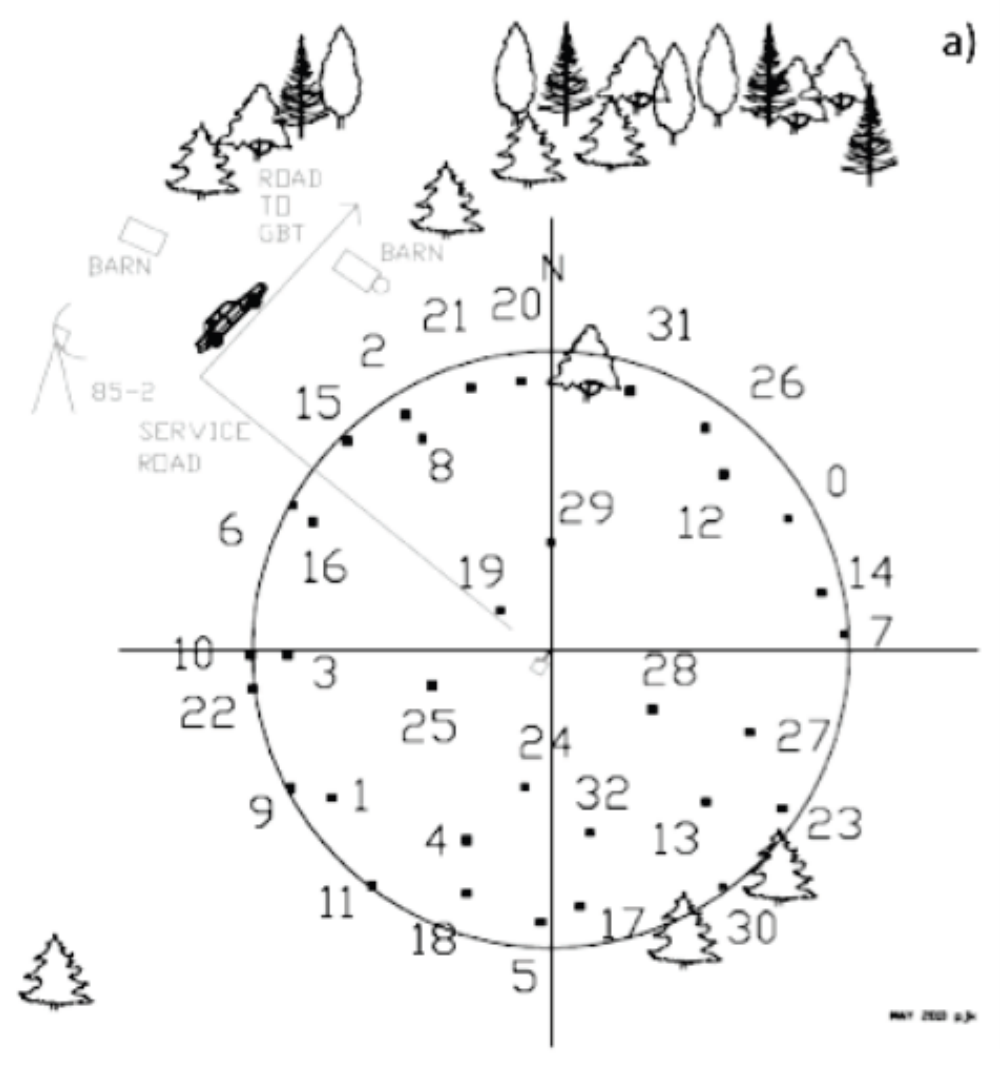

Fig. 2.5. - Diagram of antenna positions for PAPER-32 in Green Bank. This same minimum-redundancy configuration was later adapted for the 32-element array in South Africa. 
and bandpass. RFI excision is also done right away before channels are averaged to make a more manageable data size. More than $40 \%$ of the data in Green Bank is lost due to aircraft traffic control, emergency communication systems, and digital television from Richmond, VA (Parsons et al. 2010).

The heart of the aipy calibration package is the visibility equation (Eqn 1.25). To build an interferometer essentially from scratch, the basic tools needed to calibrate an array and phase to a source are the positions of the antennas, the exact time, and the frequency of the measurements. With time being regulated by the computer (which is calibrated to an NTP server) and frequency being recorded, the biggest challenge in the early stages was to pin down the exact locations of the antennas with respect to each other, as well as the cable delays. Such delays were regulated by improved methods of cutting the cables in the field to the exact length, but a difference of only a few inches was comparable to a few nanoseconds of delay.

As described in Section 2.2, the positioning of the array had a crude beginning. With the antique theodolite and the Green Bank surveying equipment, accuracy of positions was still only a few feet at best, despite great pains to insure quality measurements of angle and distance. These showed up as errors of tens of nanoseconds in the visibility data and had to be corrected in order to continue with data processing.

Initially, a simple model of the sky was used with Cygnus A, Cassiopeia A, and other bright sources to get a handle on antenna positions. A visibility model was made and used a least-squares fitting routine to iterate on the best antenna positions and delays. However, it was easy to fall into a false minimum, so this process had to be done carefully, using just a few antennas at a time over small frequency ranges, only pulling in more antennas as fitting progressed. This challenging process could take months for each configuration, thus a GPS method of finding new positions 
was developed to alleviate this process. Though restricted by RFI constraints at both array sites, once in place, these new positions were accurate to within several nanoseconds, thus affording the precision that PAPER needed to quickly turn around a new dataset.

This was demonstrated during the 64-antenna deployment in South Africa in July 2011. Though the size of the data was unmanageable with one laptop in the field, several were used in parallel, each working on a section of the array, and were able to calibrate and phase accurately after just a day or so of processing. The improved position accuracy of the GPS helped, leaving just the delays to be fit and images of Centaurus A to be made within a few days of array completion (see Figure 2.6).

At this point, CASA was well enough developed for use by the PAPER team. Although it does not have the proper tools for analyzing the power spectrum as needed for the EoR experiment, the calibration and imaging routines are much more intuitive for long-time AIPS users and can handle the large fields of view used by PAPER. Forthcoming ionosphere work will focus on results from imaging with the CASA package. 


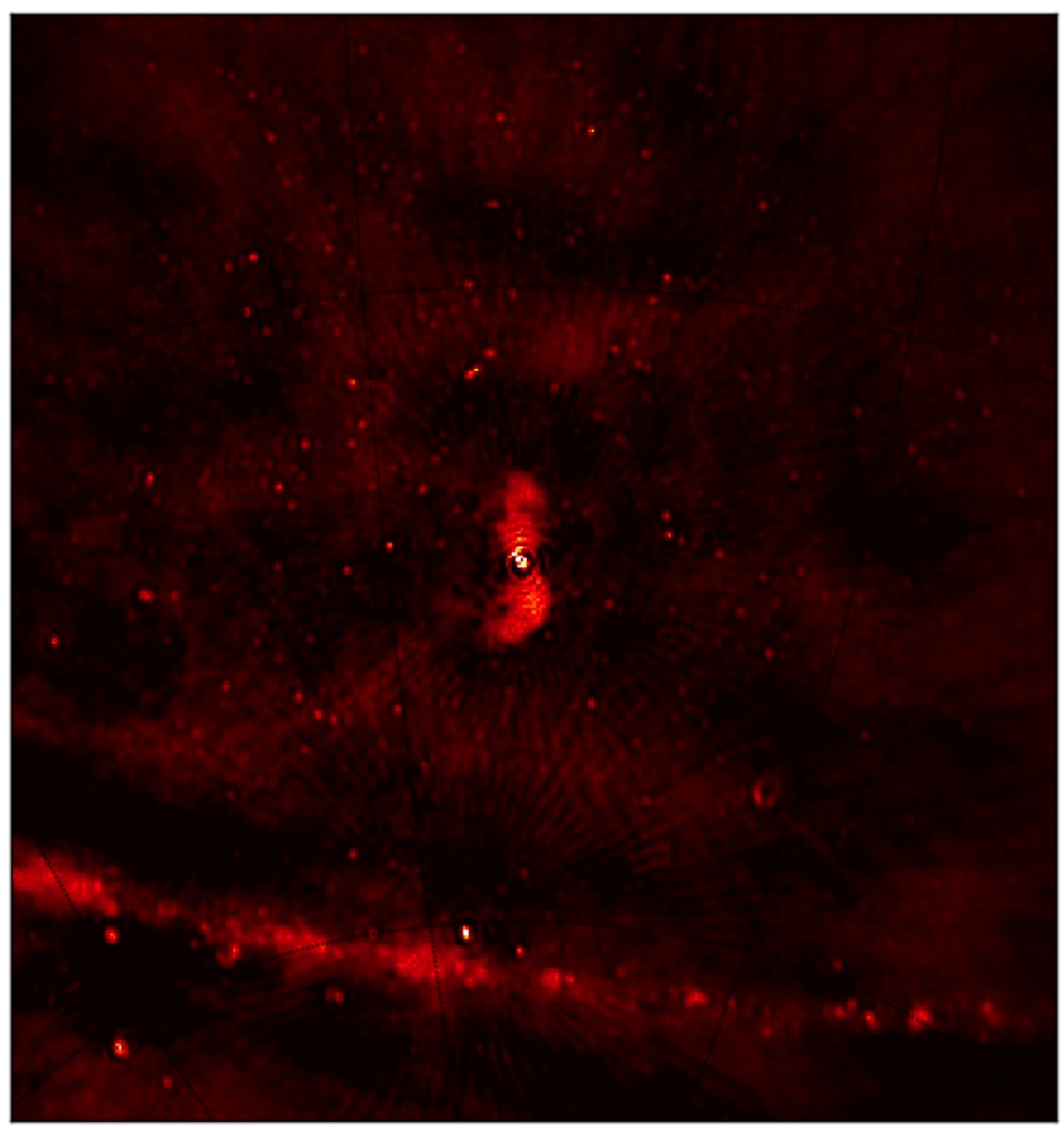

Fig. 2.6. - The double-lobe structure of Centaurus A is clearly visible, along few a few point sources and the Galactic Plane, after initial calibration with the 64-element array in South Africa in July 2011. 


\section{Chapter 3}

\section{Total Power Measurement}

\subsection{Introduction}

In section 1.2, Equation 1.11 related the power of a radiometer to the system temperature. In order to get the system temperature, add up the noise temperatures of the instrument along with that of the source, $T_{s r c}$,

$$
T_{\text {sys }}=T_{\text {inst }}+T_{\text {sky }}+T_{\text {src }}+\ldots
$$

which is measured in Kelvin. In addition to the elements of the telescope that were described in Chapter 2, the ambient sky has some noise to it which varies with frequency, at least from the Cosmic Microwave Background (CMB). $T_{s r c}$ is often a few orders of magnitude less than the other factors. However, for astronomy in the $150 \mathrm{MHz}$ range, emission from the Milky Way Galaxy dominates the sky noise, as seen in Figure 1.7. The sky was mapped at $408 \mathrm{MHz}$ by Haslam et al. (1981, 1982). This can be extrapolated to PAPER wavelengths in MHz using 


$$
T_{\nu}=T_{408}\left(\frac{\nu}{408}\right)^{-2.55}
$$

(Bridle 1967; Sironi 1974; Roger et al. 1999). Note that this map and relation will be useful in determining what parts of the sky will be best for studying EoR. If $T_{\nu}$ exceeds $T_{\text {sys }}$, then improving the system temperature of the hardware will not improve the sensitivity. This map also allows for a fixed temperature scale on the correlator output and the measurement of the receiver temperature for a single element.

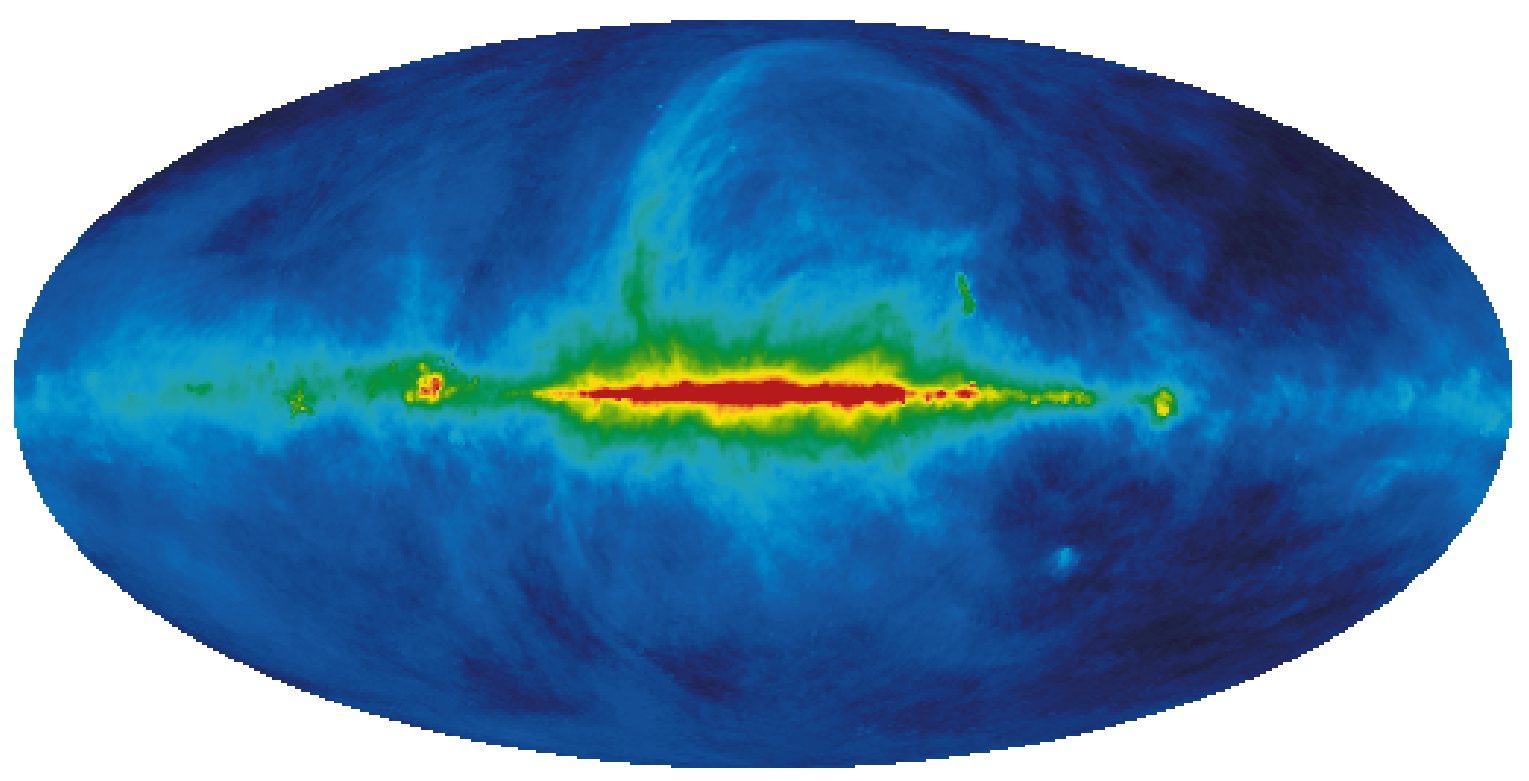

Fig. 3.1.- The Galactic Plane as seen at $408 \mathrm{MHz}$ by the Haslam All-Sky Survey. This image is from a HealPix map created by NASA's Legacy Archive for Microwave Background Data Analysis.

For the initial measurements of the system temperature, the minuscule source (EoR) and CMB signals can be ignored. The Galactic signal is synchrotron emission, created as highly energetic charged particles spiral around magnetic fields lines throughout the Galaxy. The synchrotron spectrum is partly determined by the energy distribution of charged particles in the interstellar medium and has been shown 
to have a spectral index of approximately -2.55 at these frequencies (Lawson et al. 1987). This means that the brightness increases as observations move to lower frequencies. Pathfinder EoR experiments will be avoiding areas of the sky with strong synchrotron emission from the Galaxy. However, it can be used as a tool for calibration.

\subsection{Method}

In order to determine the noise temperature of the array, the method used by Alan Rogers to calibrate the Deuterium Array (Rogers 2004) was used. This method looks at the data from one individual antenna at a time. The autocorrelations for an interferometer are equivalent to the total power measured in the system by the Wiener-Khinchin theorem, so total power data could be collected from the whole array at once through the correlator.

In this method, the system temperature is separated into the bright Galactic component, $T_{g a l}$, and the instrument component, $T_{\text {inst }}$. This also includes the overall gain of the instrument, $G$. Therefore,

$$
P_{\nu}=G k\left(T_{g a l}+T_{\text {inst }}\right)
$$

However, $T_{\text {inst }}$ is dominated by the noise temperature of the very first element in the chain, the balun, as shown by the Friis formula for calculating the noise in a series of elements 1 through $N$ (Kraus 1966),

$$
T_{\text {total }}=T_{1}+\frac{T_{2}}{G_{1}}+\frac{T_{3}}{G_{1} G_{2}}+\ldots+\frac{T_{N}}{G_{1} \ldots G_{N-1}}
$$

Therefore, $T_{\text {inst }}$ has been replaced with $T_{b a l}$ in Equation 3.3 and in future deriva- 
tions.

With data selected in a narrow band around $162 \mathrm{MHz}$, Equation 3.2 becomes

$$
T_{162}=T_{408}\left(\frac{162 \mathrm{MHz}}{408 \mathrm{MHz}}\right)^{-2.55}
$$

$T_{162}$ is calculated at every position in this way. This extrapolated map is then smoothed to a lower resolution of 30 arcminutes, or the size of the PAPER beam. The model is modified so that $300 \mathrm{~K}$ is inserted at every point with an azimuth below 6 degrees, representing the ground temperature, since the antenna beam has some reception over the entire sphere.

This model sky must then be convolved with the reception pattern of the antenna at a chosen frequency, in this case, $162 \mathrm{MHz}$. This beam model was calculated in CST Microwave Studio (see Figure 2.3). The model lists gain $(D)$ per degree altitude and azimuth. Given a certain time on a particular day this can be translated to hour angle $(h)$ and declination $(\delta)$ on the sky using various routines in the IDL Astronomy User's Library ${ }^{1}$.

$$
T_{\text {gal }}(t)=\frac{\sum T_{162}(h, \delta) D(h, \delta)}{\sum D(h, \delta)} .
$$

With that, Equation 3.3 can be written as a simple linear equation

$$
P_{\nu}=a T_{g a l}+b
$$

where $a$ is equal to $G k$ and $b / a$ is $T_{b a l}$. Thus, a linear fit of the power collected by the antenna and the Galaxy model would provide the instrumentation noise temperature and the gain of that individual array element.

\footnotetext{
${ }^{1}$ http://idlastro.gsfc.nasa.gov
} 
Data for this experiment were taken in September 2006 with eight antennas in Green Bank, West Virginia, home of the National Radio Quiet Zone and PAPER's test installation.

\subsection{Data and Analysis}

Figure 3.2 shows the measured power over a full day of data along with the corresponding model. Note that both are plotted in terms of Local Sidereal Time as the model repeats every 23 hours and 56 minutes. Units of power were, at this stage, displayed in digital units and not yet converted to a power in milliWatts.
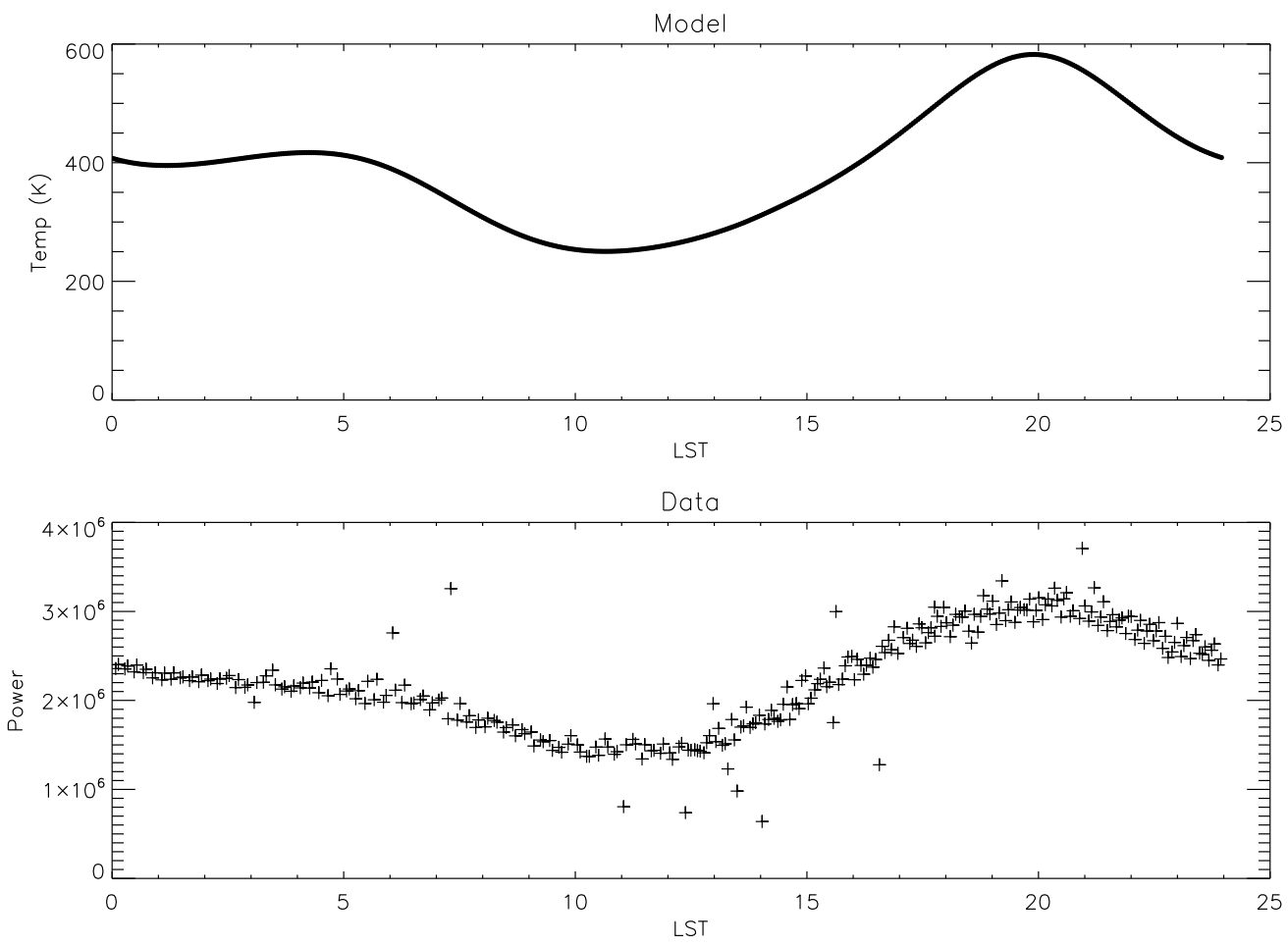

Fig. 3.2.- Top: Model of single-antenna observations using the extrapolation of the Haslam sky map and the simulated antenna beam. Bottom: Power data from antenna 1 of 8 in Green Bank in September 2006. Both are shown as a function of Local Sidereal Time. 


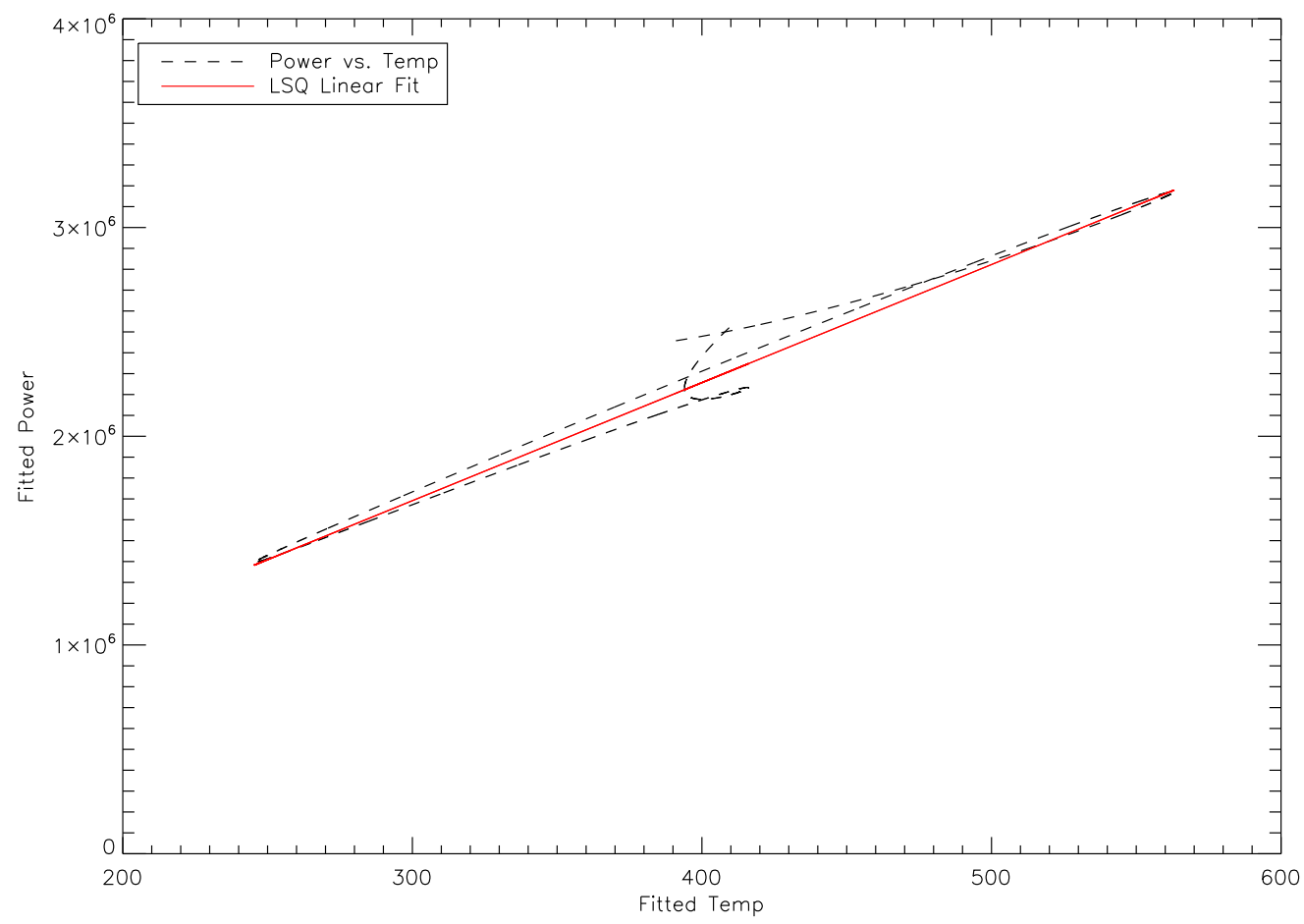

Fig. 3.3. - The black dashed line plots the model temperature versus the total power from the data for one antenna over a 24-hour period, matched up in time. The red solid line is the expected relationship between the model and data given the fitted set of parameters, gain and instrument temperature.

The temperature and power are fit to Eqn. 3.7 using the IDL function poly_fit.pro. The coefficients are then used to predict what the power should be given the temperature model. However, when the actual power is plotted against the model, the results are not linear, but instead take on a skewed shape in this space (see Figure $3.3)$.

In addition, the fitted $T_{b a l}$ is negative, which is clearly unphysical. The situation is more obvious when the power is predicted from the model temperature using the fit parameters, or the sky temperature is predicted from the power data (see Figure 3.4). Similar behavior was noticed for all eight antennas in the array.

The leading hypothesis was that the ambient temperature was affecting the gain of 

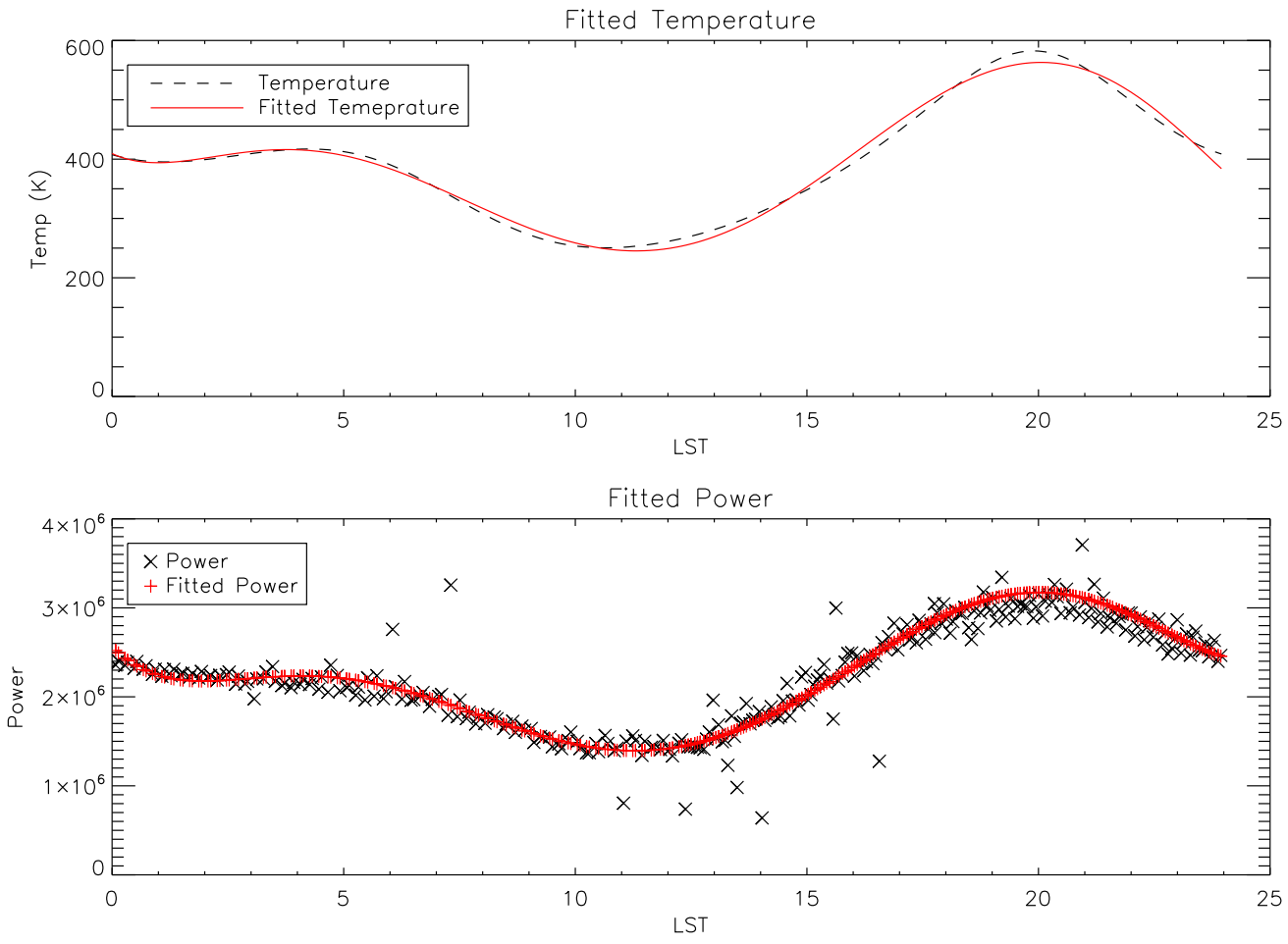

Fig. 3.4. - Top: Model temperature and temperature predicted from the data. Bottom: Actual power data and power predicted from the model. This shows, in addition to Figure 3.3, that the fitted parameters are not precise over the entire day range.

the balun. The balun is enclosed in the supporting "neck" of the antenna which tends to get very hot when the antenna is in sunlight. In particular, a rise in the ambient temperature would mean a lower gain. To test this hypothesis, the corresponding ambient temperature data was collected from a nearby weather station on site at Green Bank, half a mile northeast of PAPER. Introducing a new temperature-dependent gain term, $G_{1}$, and the ambient temperature, $T_{a m b}$, the IDL function curvefit was used to determine the gains and noise temperature from

$$
P_{\nu}=\left(G_{0}+G_{1} T_{a m b}\right)\left(T_{b a l}+T_{g a l}\right)
$$

This experiment was repeated with the eight-antenna array in February of 2007. 
Figure 3.5 gives an example of the results. Although the fit is much improved, as seen in the third row, with the inclusion of the temperature-dependent gain, the resulting $T_{b a l}$ is $52 \mathrm{~K}$, a physical answer, but much below the laboratory measurement of the noise temperature of $110 \mathrm{~K}$ (Parashare, private communication).
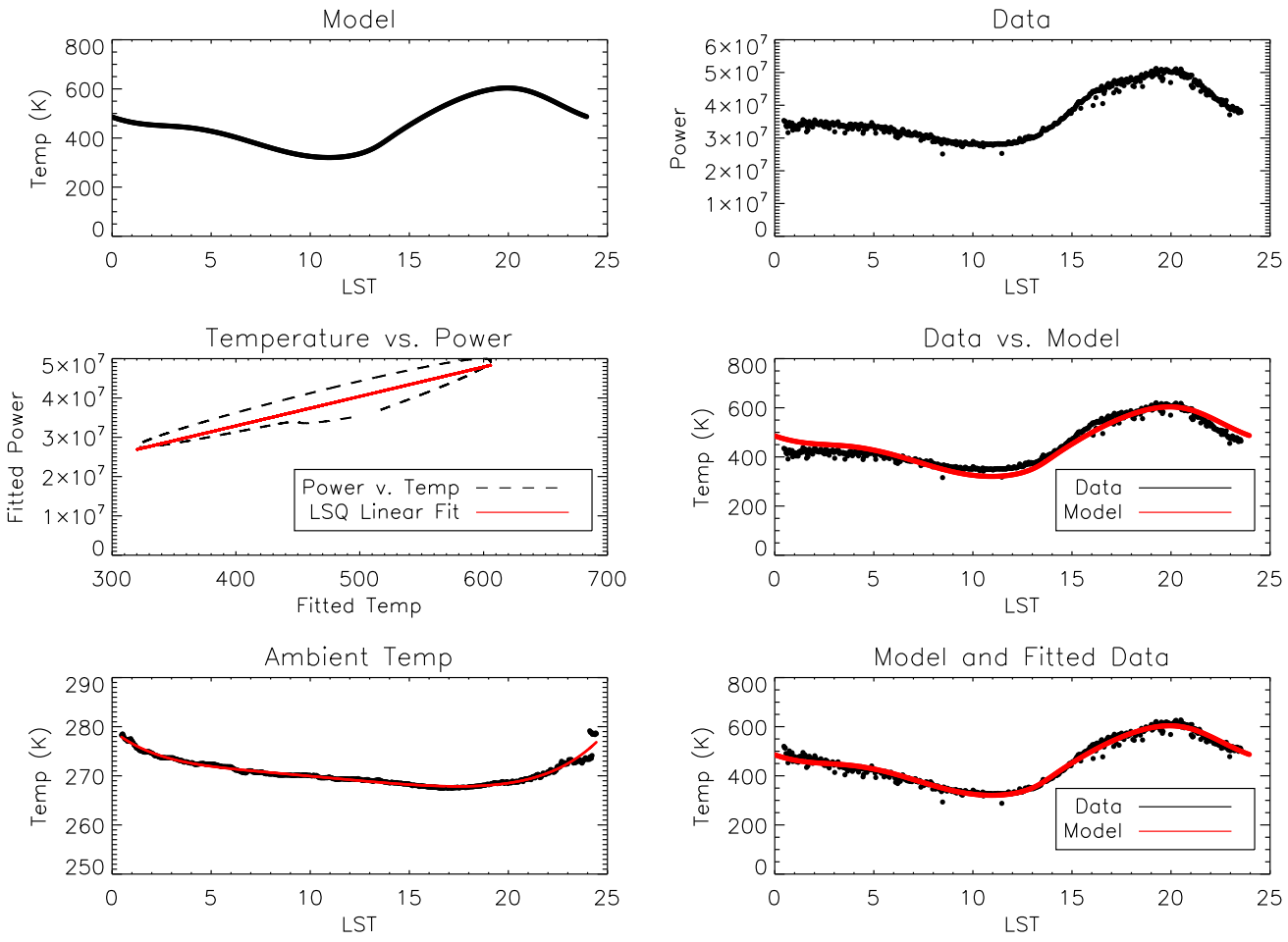

Fig. 3.5.- Top left: Model. Top right: Data. Middle left: Result of linear fit. Middle right: Model and power extrapolated with linear fit. Bottom left: Ambient temperature with polynomial fit. Bottom right: Model with power extrapolated from fit to Eqn 3.7.

\subsection{Engineering Work}

The ambient temperature fit only goes so far toward measuring the system gain and $T_{b a l}$. Clearly, a more accurate system is needed. UVa Engineering graduate student Chaitali Parashare took on the task of measuring temperature dependent 
gains for every element in the system in the lab with a temperature control box, with indoor temperature fluctuations, and with outdoor fluctuations. The balun, cable, and receivers of one antenna were fitted with temperature sensors so that an accurate ambient temperature could be found and the fluctuations of the gain accurately measured. The results of this work are available in Parashare (2011). For subsequent work with the array, the temperature of a single antenna was used to remove the time-variant gain to first order. This "gainometer" also would not see the sky, but was attached to a load which also had a temperature sensor built in.

\subsection{Conclusion}

It is expected that the field values of the noise temperature of the antenna system is similar to that which was measured in the laboratory at 110 K. Some data sets already indicate a fit near this value, though precise measurements could not be made without the meticulous observations of gain changes with temperature in the lab by Chaitali Parashare. In addition, the gainometer has added a crucial component to the overall calibration scheme of PAPER. An important issue with PAPER data collection was discovered early on and solved to ensure precise measurements. This project also demonstrates the advantage of having collaborators from different disciplines work in tandem to discover and solve a problem in radio astronomy. 


\section{Chapter 4}

\section{Effects of the Ionosphere}

\subsection{Radio Astronomy and the Ionosphere}

The ionosphere is the layer of the Earth's atmosphere that has been ionized by solar radiation. The TEC (total electron content) is an important quantity that measures the total number of electrons along a line of sight in units of TECU, or $10^{16}$ electrons $\mathrm{m}^{-2}$.

The layers of the ionosphere are labelled D, E, F1, and F2, with approximate heights and electron densities shown in Figure 4.1. Densities are generally higher during the day than at night and during solar maximum as opposed to solar minimum. The global distribution of electrons around the ionosphere changes daily, such that the area of highest ionization is at the subsolar point. Thus, the changes in the TEC are also seasonal for a particular location on Earth. The structure of the ionosphere is rather regular at middle and equatorial latitudes, compared to the polar regions where the Earth's magnetic field plays a more important role. However, the equatorial anomaly introduces a trough in the TEC of the $\mathrm{F}$ layer at the magnetic equator as plasma flows to higher latitudes as a current. These large scale ionospheric structures 
can be seen in Figure 4.2.

Irregularities and density fluctuations can be on scales from 1 to $1000 \mathrm{~km}$ in size (Hinder \& Ryle 1971). Medium-sized irregularities (10-400 km) are known as traveling ionospheric disturbances (TIDs) and have periods $<90$ minutes, whereas small-scale irregularities (1-10 km) occur primarily at night and cause radio scintillation for lowfrequency telescopes. Large-scale structures $(\sim 1000 \mathrm{~km})$ include the "wedge" present at dawn and at dusk during rapid periods of ionization and recombination. (Smallest structures may "never" be corrected for in interferometric data, according to Kassim et al. (2007).)

As the 2012-2013 solar maximum approaches, observatories will be on the lookout for SIDs, or sudden ionospheric disturbances. A sudden enhancement in H-alpha emission from the Sun is called a solar flare, and it is often accompanied by an increase in solar x-rays. This produces a sudden increase in ionization in the D layer of the ionosphere over the course of minutes, which then slowly recovers back to normal densities over a few hours.

It is useful to first look back on what is known about the ionosphere in regard to radio astronomical observations. Hinder \& Ryle (1971) explored this issue as it became relevant with the advent of aperture synthesis and the building of radio interferometers that had baselines that were tens of kilometers long. They realized that the ionosphere creates a path length increase along the line of sight to an individual receiving element of

$$
\Delta L \approx-4.5 \times 10^{-16} \lambda^{2} T E C
$$

where $\lambda$ is the wavelength in meters and TEC is the total electron content along the line of sight in electrons per meters squared. It was already known at this time 


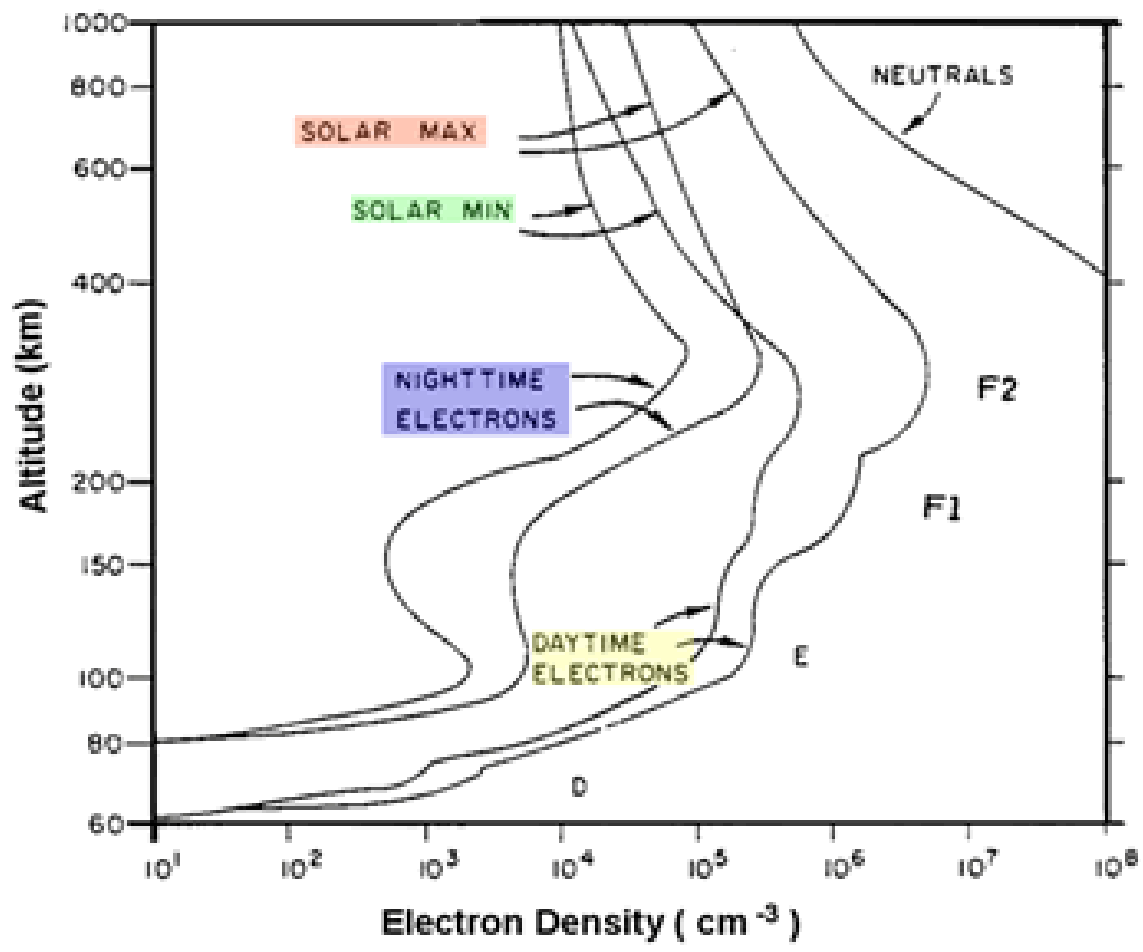

Fig. 4.1. - Electronic density as a function of elevation for daytime, nightime, solar maximum and solar minimum periods. The F2, F1, E and D layers are indicated. From Peter Guest at http://www.weather.nps.navy.mil/ psguest/ 


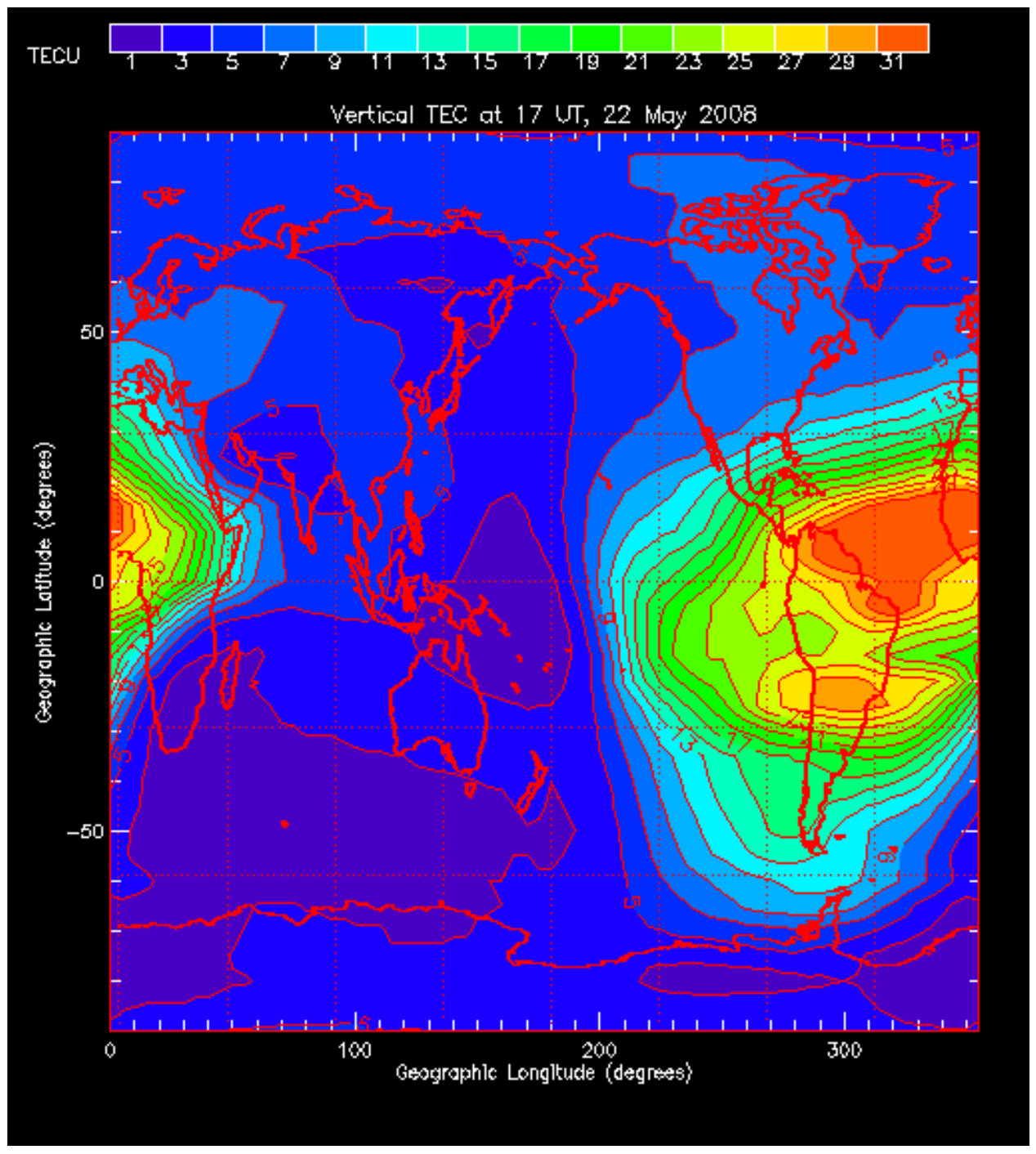

Fig. 4.2.- Global TEC distribution from by the Australian Government Radio and Space Services as measured by GPS satellites sending signals to ground-based receivers at several frequencies. The continents are outlined in red and the color map indicates the total electron content in TECU. The area of highest density occurs on the daytime side of the Earth, following the subsolar point as ultraviolet solar radiation ionizes those regions of the atmosphere. The aforementioned equatorial anomaly can be seen as a trough in density over South America. 
from Faraday rotation measurements with Earth-orbiting satellites that irregularities in the TEC existed on size scales ranging from 1 to $1000 \mathrm{~km}$. In fact, the ionosphere has been probed by various methods to determine the size and behavior of irregularities, such as ionospheric sounding, Doppler shift, and Faraday rotation (Ratcliffe 1972). Hinder \& Ryle determined that large scale TIDs (traveling ionospheric disturbances) were rare and that large gradients near sunrise could be avoided by simply not observing during these times. However, smaller irregularities would pose a calibration issue, and the overall curvature of the ionosphere would begin to affect baselines larger than $100 \mathrm{~km}$. A numerical analysis of a model eight-element interferometer with a maximum baseline of $10 \mathrm{~km}$ shows that the sidelobes introduced in the synthesized beam are $3 \%$ of the main beam, but the curvature effects limit the array size to a few kilometers if accurate corrections are not implemented using bright point sources. They recommend that aperture arrays restrict their observing frequency coverage to minimize the effects of the ionosphere at low frequencies and similar effects of the troposphere at high frequencies.

The Very Large Array (VLA) ${ }^{1}$ is one such example of a successful aperture synthesis telescope that became productive for science in the centimeter regime. However, just a few years after its official inauguration, Perley \& Erickson (1984) proposed a low-frequency telescope for the site in New Mexico. The memo details a calibration strategy that rejects data from time periods with obviously bad scintillation and uses models and maps from higher frequencies observations to calibrate within the isoplanatic patch. However, this would limit the field of view to just a few degrees around the phase center. The isoplanatic patch size is essentially the Fried parameter, determined by the turbulence of the refractive layer which is, in the case of low frequency radio astronomy, the ionosphere. At centimeter wavelengths, the isoplanatic patch is

\footnotetext{
${ }^{1}$ https://science.nrao.edu/facilities/evla
} 
typically larger than the field of view. However, the isoplanatic patch shrinks in size with increasing wavelength as the excess path length through the ionosphere is proportional to $\nu^{-2}$. (Note that in the optical regime, the isoplanatic patch is typically much smaller, several arcseconds across, and is due to turbulence in the troposphere.)

Radio telescopes working at low frequencies could continue to be either restricted by this few degree of sky limitation or try and use another method to determine the structure of the ionosphere. Spoelstra (1983) demonstrated that some corrections could be made with ionosonde data from a ground station and several satellites when combined with $608.5 \mathrm{MHz}$ observations with the Westerbork Synthesis Radio Telescope $^{2}$. Though astronomical source refraction was reduced, the results were ultimately limited by the availability of ionosonde data.

As global positioning system (GPS) technology became ubiquitous, these satellites could also be used to probe the TEC along lines of sight near astronomical sources of interest. From 1995 to 1999, experiments were run at $327 \mathrm{MHz}$ with the VLA and four GPS receivers that were installed on site to probe the local ionosphere (Erickson et al. 2001). Real-time measurements of the total electron content towards the satellites were applied to a model ionosphere which started as a simple uniform layer. GPS data provided pierce points through the ionosphere $1000 \mathrm{~km}$ apart, thus correcting for the largest irregularities. Effects on a scale of $100 \mathrm{~km}$ could only be corrected for in the isoplanatic patch $\left(\sim 4^{\circ}\right)$ around each satellite, so that many more ground receivers would be needed for this method to be useful for observations. This was deemed impractical for the VLA at the time.

A $74 \mathrm{MHz}$ receiver system was implemented in 1998 (Kassim et al. 2007). Since refraction due to the ionosphere scales as $\lambda^{2}$, challenges to calibration would be even more severe for this instrument than for observations near $150 \mathrm{MHz}$. In addition,

\footnotetext{
${ }^{2}$ http://www.astron.nl/radio-observatory/astronomers/wsrt-astronomers
} 
the $13^{\circ} \mathrm{FWHM}$ primary beam would have multiple isoplanatic patches in each field of view. For the prototype of this system (Kassim et al. 1993), simultaneous measurements were made at $74 \mathrm{MHz}$ and $330 \mathrm{MHz}$, and ionospheric fluctuations were measured at the higher frequency and extrapolated to the lower frequency in order to make self-calibration and imaging possible. With the full system in place, however, a new calibration technique was developed so that the $74 \mathrm{MHz}$ band could truly become a "facility-level" instrument.

Kassim et al. (2007) demonstrates various effects of the ionosphere using the bright radio source Virgo A. A typical 8-hour observation shows large gradients at dawn, nighttime scintillation, and large TIDs in the phase differences between antennas of the array. (See Figure 4.3.) These effects were more exaggerated along longer baselines and resulted in source wander on a scale of several arcminutes. Imaging fields of view that were larger than the isoplanatic patch using traditional self-calibration methods showed distortions, position offsets, and loss of signal to noise away from the astronomical calibrator source. Self-calibration in radio interferometry is the iterative process by which phase and amplitude errors are removed from the visibilities, using the target source itself as a model, rather than an external calibrator. This process has served well for small fields of view, but a single phase offset is inadequate to correct for distortions over a larger field of view. In order to cope with these effects, a "field-based" calibration strategy was implemented using snapshot images tied to an astrometric grid, usually from a catalog at higher frequencies, such as the NVSS (Condon et al. 1998). By mapping the position offsets around the field of view, a phase screen model of the ionosphere was created assuming a thin-layer ionosphere and low-order Zernike polynomials to model the irregularities. This improved the job of removing distortions and allowing identification of point sources across the field of 
view, leading to the VLA Low-frequency Sky Survey (VLSS), which catalogued over 70,000 sources (Cohen et al. 2007).

Field-based calibration is useful in a certain regime where the array is relatively small in comparison to the physical extent of ionospheric irregularities. These four "ionospheric calibration regimes" as described by Lonsdale (2005) are diagrammed in Figure 4.3. The first two regimes have telescopes with small fields of view, and thus work well with the traditional self-calibration. The VLA $74 \mathrm{MHz}$ system was in regime 3 , in which the field of view is larger than the size scale of the irregularities. PAPER is an extreme example of this regime as the zenith-pointing antennas have a full-width half-max of $\sim 45^{\circ}$. Much larger arrays, such as LOFAR, will reside in regime 4 and will have additional challenges due to the large array extent. Typical EoR experiments, however, do not require high spatial resolution, and so these will remain in regime 3 . As the PAPER field of view is so large $\left(\mathrm{FWHM} \sim 45^{\circ}\right)$, however, it remains to be seen to what level field-based or related calibration schemes such as SPAM (Intema et al. 2009) can be implemented, and whether there are any unforeseen technical challenges to imaging and foreground removal.

The overall statistics of ionosphere density fluctuations at the size scales that affect the EoR measurement are unknown and should be characterized before predictions can be made. Cohen \& Rottgering (2009) began identifying statistical patterns using the VLSS and a technique called differential refraction. This technique takes a pair of astronomical sources and tracks their distance from one another rather than from their absolute positions so that instrumental phase delay does not contribute. From analyzing hundreds of hours of observations for the VLSS, they found that increasing refraction due to decreasing elevation could be easily corrected by a thin-shell model. Daytime observations showed significantly more variation than nighttime observa- 


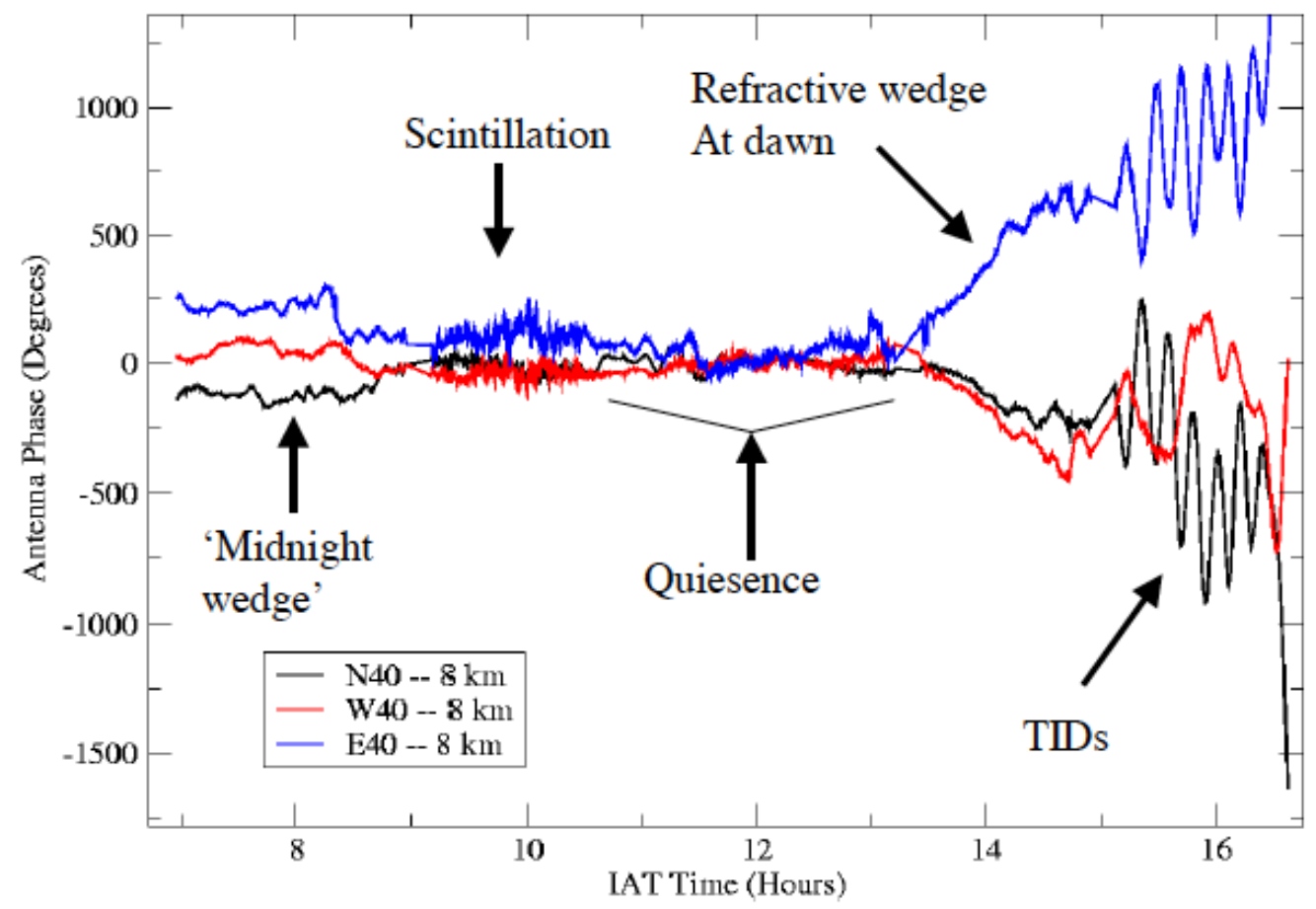

Fig. 4.3. - Phase of three antennas relative to a central antenna during an approximately 8 hour observation of Virgo A illustrating many of the ionospheric phenomena typically observed at the VLA. Ionospheric disturbances on small, medium, and large size scales are seen, with TID's happening during the daytime, scintillation at night, and the refractive wedge at dawn which makes observations difficult if not impossible near sunrise. From Kassim et al. (2007). 


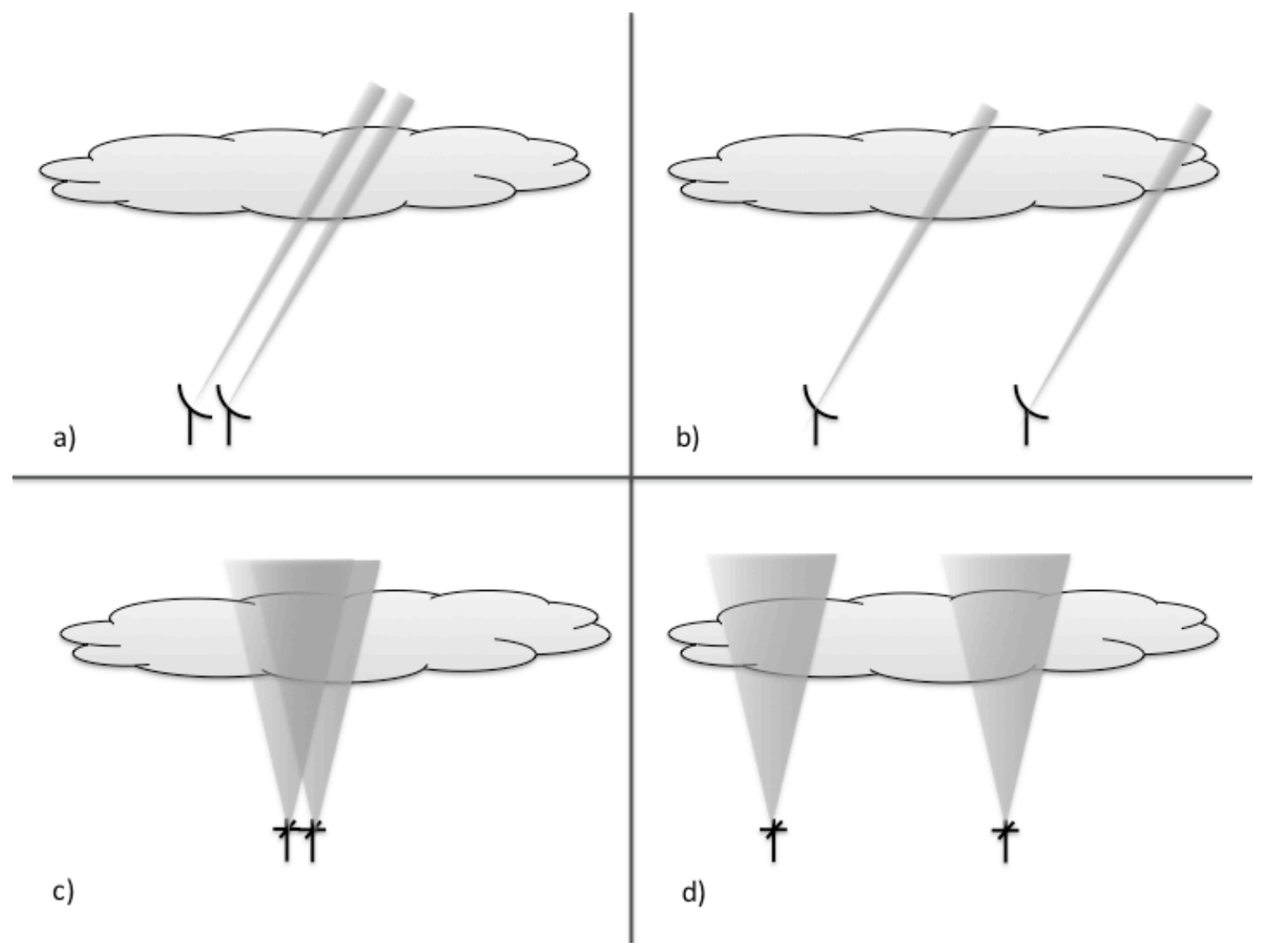

Fig. 4.4.- Cartoon of the four ionosphere calibration regimes. When the field of view is smaller than the scale of ionospheric density fluctuations, such as in cases (a) and (b), self-calibration is used without regard to the ionosphere. PAPER is an example of case (c) where the field of view encompasses several ionospheric patches, but the array is small in comparison to these. Larger observatories, such as LOFAR, will have to work in the most challenging regime (d). 
tions. Daytime observations also tended to have irregularities on larger size scales (100s km, as opposed to $10 \mathrm{~s} \mathrm{~km}$.) This work was, as the authors describe, just the "tip of the iceberg" for characterizing the ionosphere with radio astronomy datasets. More sampling must be done to correlate disturbances with time of year and time of the solar cycle. PAPER may be used to continue this monitoring as well as probe some of the largest ionospheric disturbances with its wide field of view.

\subsection{Observations}

Our initial search for ionospheric effects on PAPER data made use of the brightest sources in the Northern sky: Cygnus A, Cassiopeia A, Virgo A, and the Crab Nebula (Taurus A). Cas A and Tau A are supernova remnants in our galaxy, while Cyg A and Vir A are active galaxies. They range from 11,600 Jy to $1050 \mathrm{Jy}$ at $178 \mathrm{MHz}$ in the 3rd Cambridge Revised Catalogue (Kellermann et al. 1969). (See Table 4.1)

Since the primary beam of PAPER has a beamwidth at the half-power point of $\sim 45^{\circ}$, one of these bright sources is almost always in the beam. However, the lower the source elevation, the more attenuated the flux, as PAPER is a non-tracking telescope. More importantly, however, these bright sources can be detected with high SNR in just one scan of 10 seconds, when high in the primary beam. A single-scan snapshot with Cygnus A has a signal-to-noise ratio, as measured by the routine "imstat" in

the radio interferometer imaging software CASA, of 16,000. These short scans allow

Table 4.1: Bright Sources

\begin{tabular}{lccc}
\hline Source & RA & Dec & 3CR Flux (Jys) \\
\hline Taurus A & 053432.0 & +220052 & 1838 \\
Virgo A & 123049.4 & +122328 & 1446 \\
Cygnus A & 195928.3 & +404402 & 10900 \\
Cassiopeia A & 232327.9 & +584852.4 & 9160 \\
\hline
\end{tabular}


us to search for the smallest, fastest moving density fluctuations that are not easily calibrated by traditional methods.

In line with previous experiments described in Section 4.1, the offsets of source positions on the sky are used as a measure of refraction due to the ionosphere on these data. The change in source position is related to the differential TEC in a given direction by

$$
\frac{d}{d x} T E C=6.59 \times 10^{-5}\left(\frac{\nu}{74 \mathrm{MHz}}\right)^{2}\left(\frac{\theta}{1^{\prime \prime}}\right) \mathrm{TECU} / \mathrm{km}
$$

where $T E C$ (total electron content) is the number of electrons located along a path between two points in TEC units (TECU), or $10^{16}$ electrons $\mathrm{m}^{-2}, \nu$ is the observing frequency in $\mathrm{MHz}$, and $\theta$ is the position shift of source in a given direction, $x$. (Cohen \& Rottgering 2009).

Twenty-four hours of data were collected with a 32-antenna array in Green Bank on July 21-22, 2010 (Julian date 2455399). The full bandwidth from the correlator is $100-200 \mathrm{MHz}$ split into 1024 channels. Calibration follows the prescription first outlined in Parsons et al. (2010) using aipy. Initial steps include gain linearization to remove quantization effects introduced by the digital correlator, RFI-excision, and cross-talk removal. The RFI excision routine fits a smooth polynomial to the visibilities and removes outliers above a user-defined standard deviation limit. At the Green Bank site, several channels are lost due to digital television signals, aircraft transmissions, and local emergency radio channels. Channels below $125 \mathrm{MHz}$ and above $170 \mathrm{MHz}$ are flagged to match the receiver bandpass and to avoid a strong digital TV signal on the higher end of the band. For integrations where a particularly strong RFI source saturated the ADC input, the rest of the band "drops out," so these integrations are flagged as well. The resulting spectrum is channel averaged to 
have 256 channels over the full range. Cross-talk removal looks for and removes an additive term varying over several hours, much longer than those of the fringe rates for astrophysical sources.

Antenna positions have been carefully marked out using a GPS system to ensure an accurate starting model for calibration. Antenna delays are fit using the interferometer measurement equation, starting GPS positions, and a starting model of the sky with bright sources. Antenna positions are tweaked via this model as well. The measurement equation for a single polarization set of visibilities, $V_{\nu}(t)$ is

$$
V_{\nu}(t)=G_{\nu}(t) \sum_{n} A_{\nu}\left(\hat{s}_{n}\right) S_{\nu}\left(\hat{s}_{n}\right) e^{2 \pi i \nu\left(\vec{b} \cdot \hat{s}_{n}+\tau\right)+\phi}
$$

where $G_{\nu}(t)$ is the complex gain, $A_{\nu}$ is the primary beam and $S_{\nu}$ the source flux in the source direction $\hat{s}_{n}, \nu$ is the observing frequency, $\vec{b}$ the baseline vector separating antenna elements, $\tau$ is the electronic delay of signals to the correlator, and $\phi$ is the relative instrumental phase. Note that this is measured for all pairs of antennas, indexed with $i$ and $j$, such that $G_{\nu}(t) \equiv g_{i \nu}(t) g_{j \nu}^{*}$ and $A_{\nu}\left(\hat{s}_{n}\right) \equiv a_{i \nu}\left(\hat{s}_{n}\right) a_{j \nu}^{*}\left(\hat{s}_{n}\right)$. This measurement equation is central to the AIPY calibration package.

The positions of the four bright sources are found with a least squares fit of the visibilities after the initial calibration steps are completed. This uses the AIPY task "fitmdl" to generate visibilities from an initial model of the array and sky and fit for the source position at each integration. Before this, the ionospheric refraction angles are normalized to a standard frequency, $150 \mathrm{MHz}$.

In order to separate each source's visibilities, the delay/delay-rate (DDR) filtering method is used as described in Parsons \& Backer (2009). This is basically executed by adding a phase term to rotate the visibilities such that the phase center is at the desired source position. Then, a Fourier transform of the spectrum of each baseline 
pair is taken, creating "delay images" so that sources can be traced by their geometric delay. Sources are more fully separated when the change in the geometric delay with time, or delay-rate, is taken into account. Since the data are already phased to the source, the fringes are stopped in time, and a Fourier transform over the time axis produces "delay/delay-rate" images which is a rough approximation of the sky. A CLEAN algorithm is applied to remove effects of lingering RFI. (CLEAN refers to a set of algorithms that were first developed by Hogbom (1974) for radio interferometry by building a model from the brightest points in the image and removing the sidelobe response between each subtraction.)

This DDR method of filtering out sources is reliable for the brightest ones in the sky, but rapidly becomes less accurate with decreasing flux. Since the sources are in our field of view for several hours, the measured source fluxes are attenuated for sources lower in the beam, so that the DDR method fails to accurately separate bright sources over the entire transit time of the source. Therefore, the more reliable results shown here come from the few hours around transit for each bright source. Source positions are then fit from the visibilities using Eqn. 4.3 at every 10-second integration, using the least square method described above. The result is an offset in both right ascension and declination of each source for every integration.

\subsection{Results}

Figures 4.5 through 4.12 show the measured position offset of each of the bright sources as a function of LST (local sidereal time) and a histogram of the position offsets in each case. Data were selected around the times of transit for each source when the DDR filter was working at its best. Table 4.2 lists the parameters for the offsets and their distributions. 

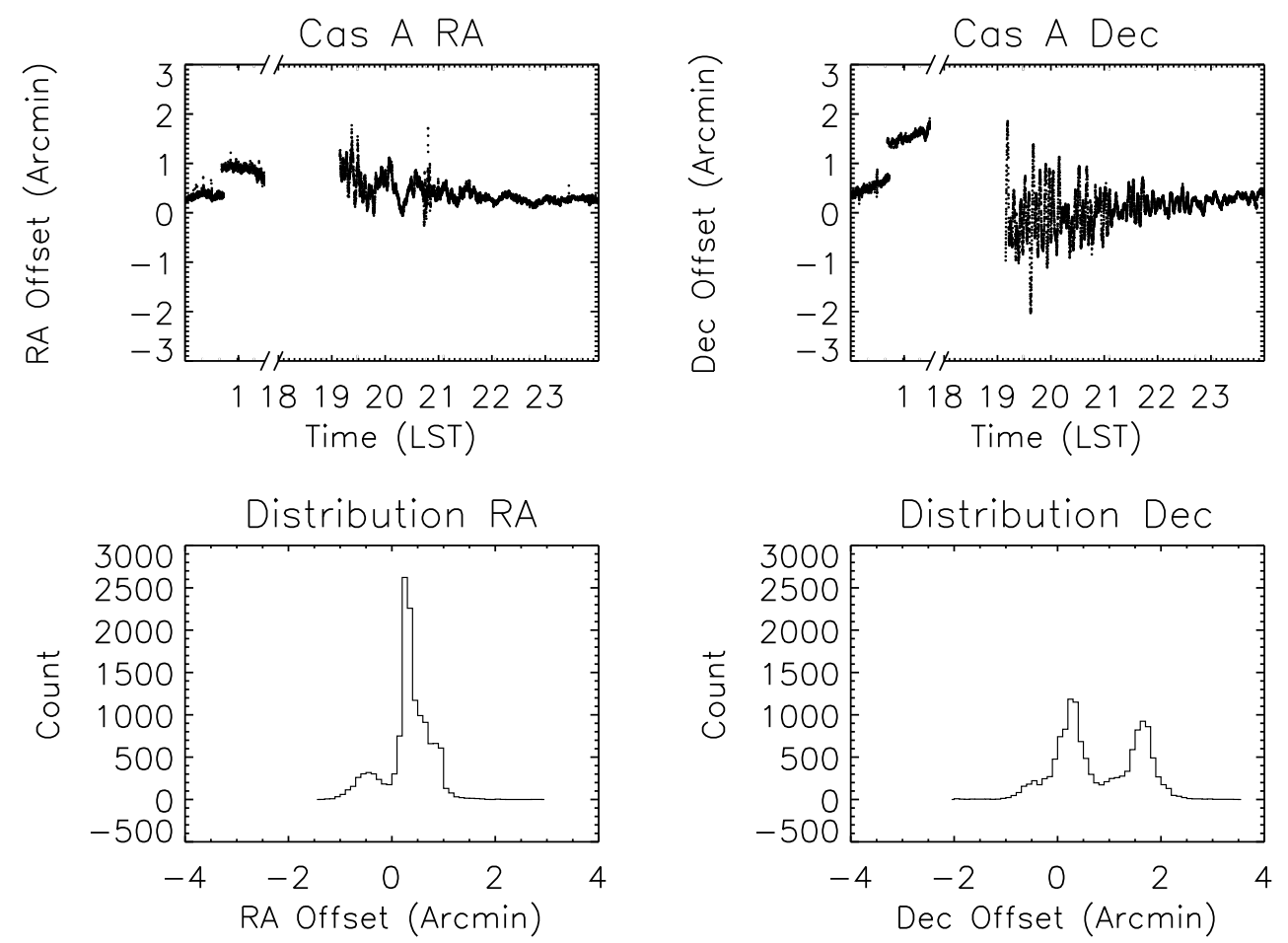

Fig. 4.5.- Top: Position offset in arcminutes vs local sidereal time for Cas A at $130 \mathrm{MHz}$, as measured by the process described in Section 4.3. Bottom: Histogram of these offsets. 

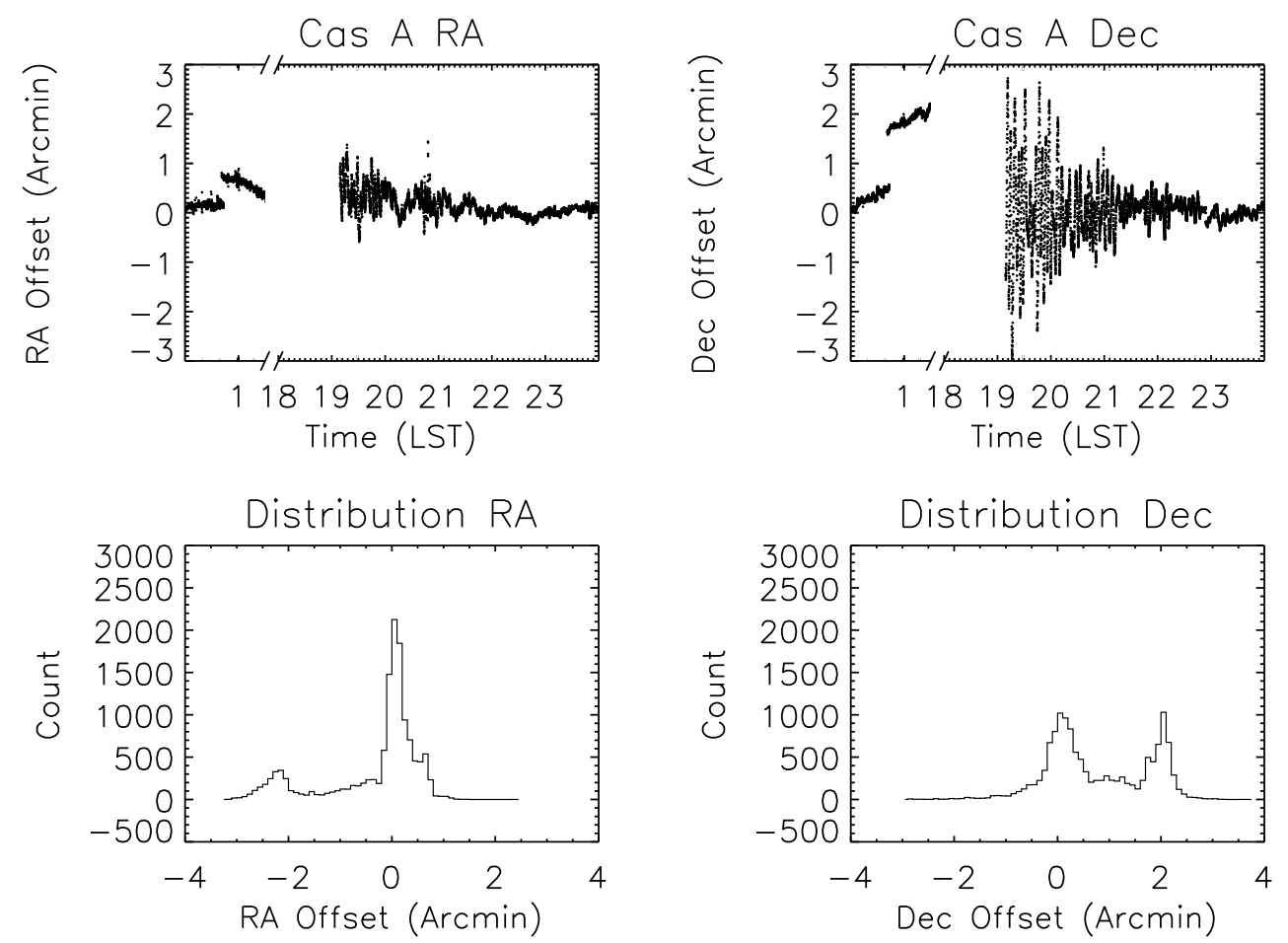

Fig. 4.6. - Top: Position offset in arcminutes vs local sidereal time for Cas A at $165 \mathrm{MHz}$, as measured by the process described in Section 4.3. Bottom: Histogram of these offsets. 

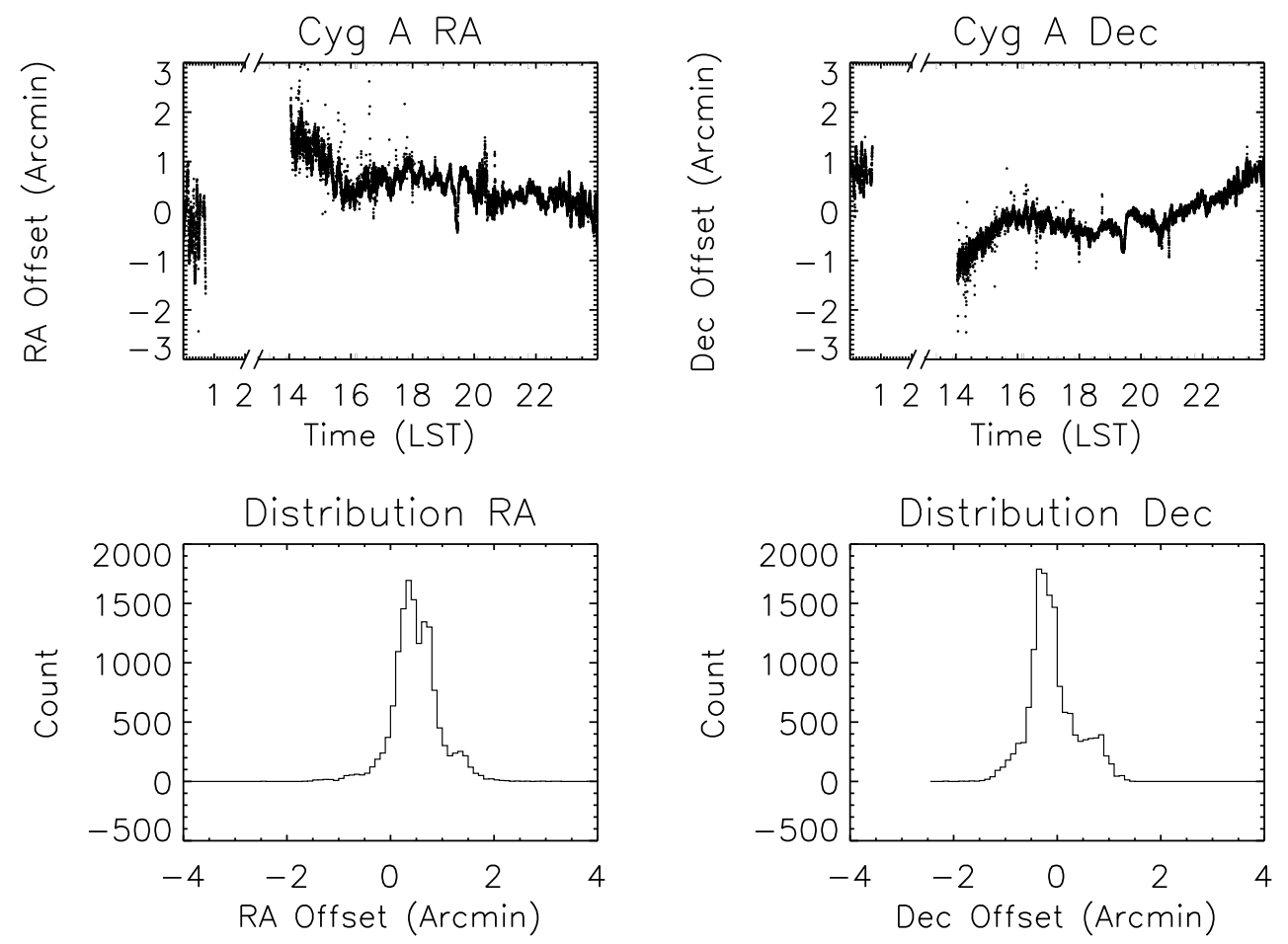

Fig. 4.7.- Top: Position offset in arcminutes vs local sidereal time for Cyg A at $130 \mathrm{MHz}$, as measured by the process described in Section 4.3. Bottom: Histogram of these offsets. 

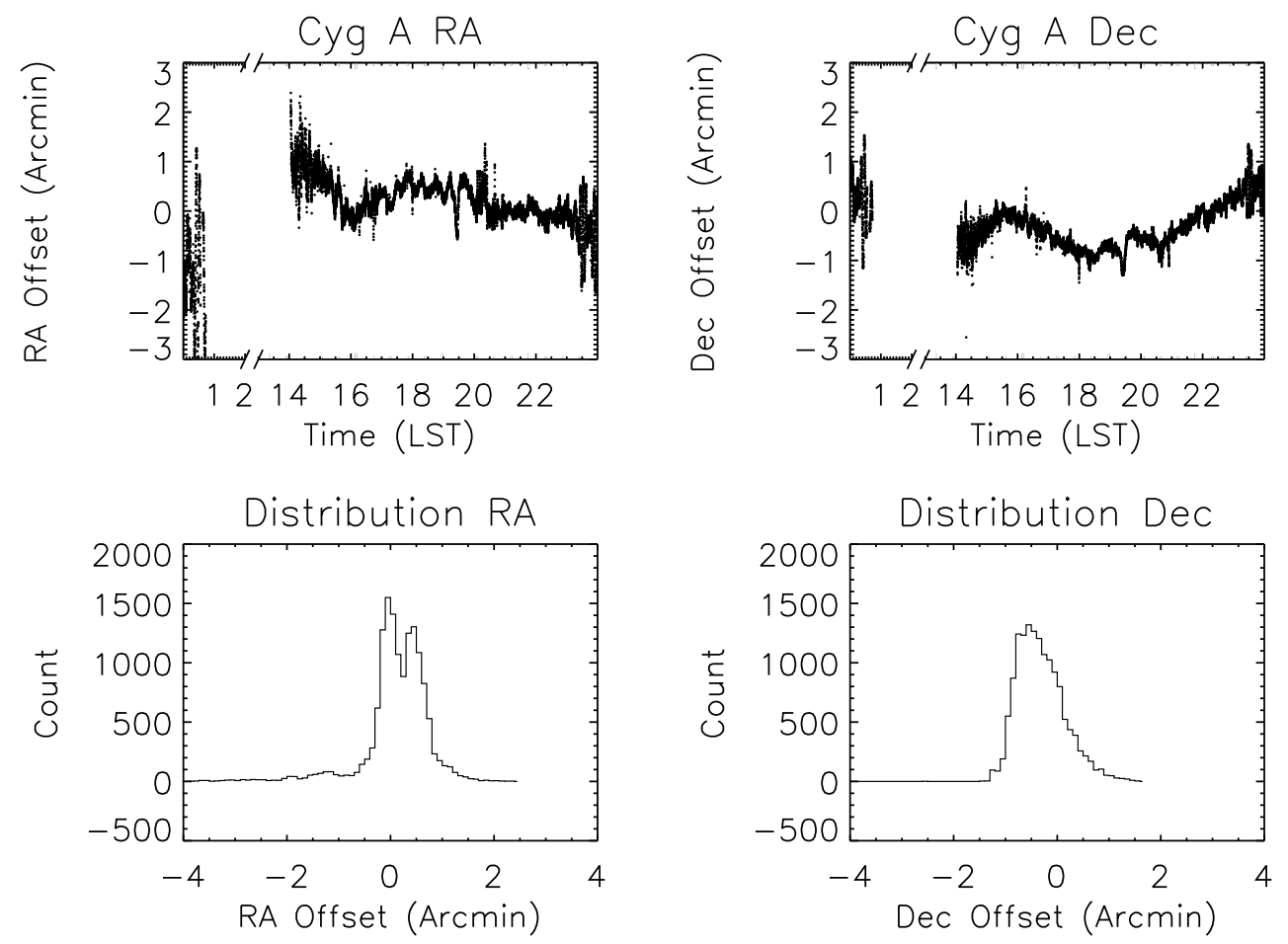

Fig. 4.8. - Top: Position offset in arcminutes vs local sidereal time for Cyg A at $165 \mathrm{MHz}$, as measured by the process described in Section 4.3. Bottom: Histogram of these offsets. 

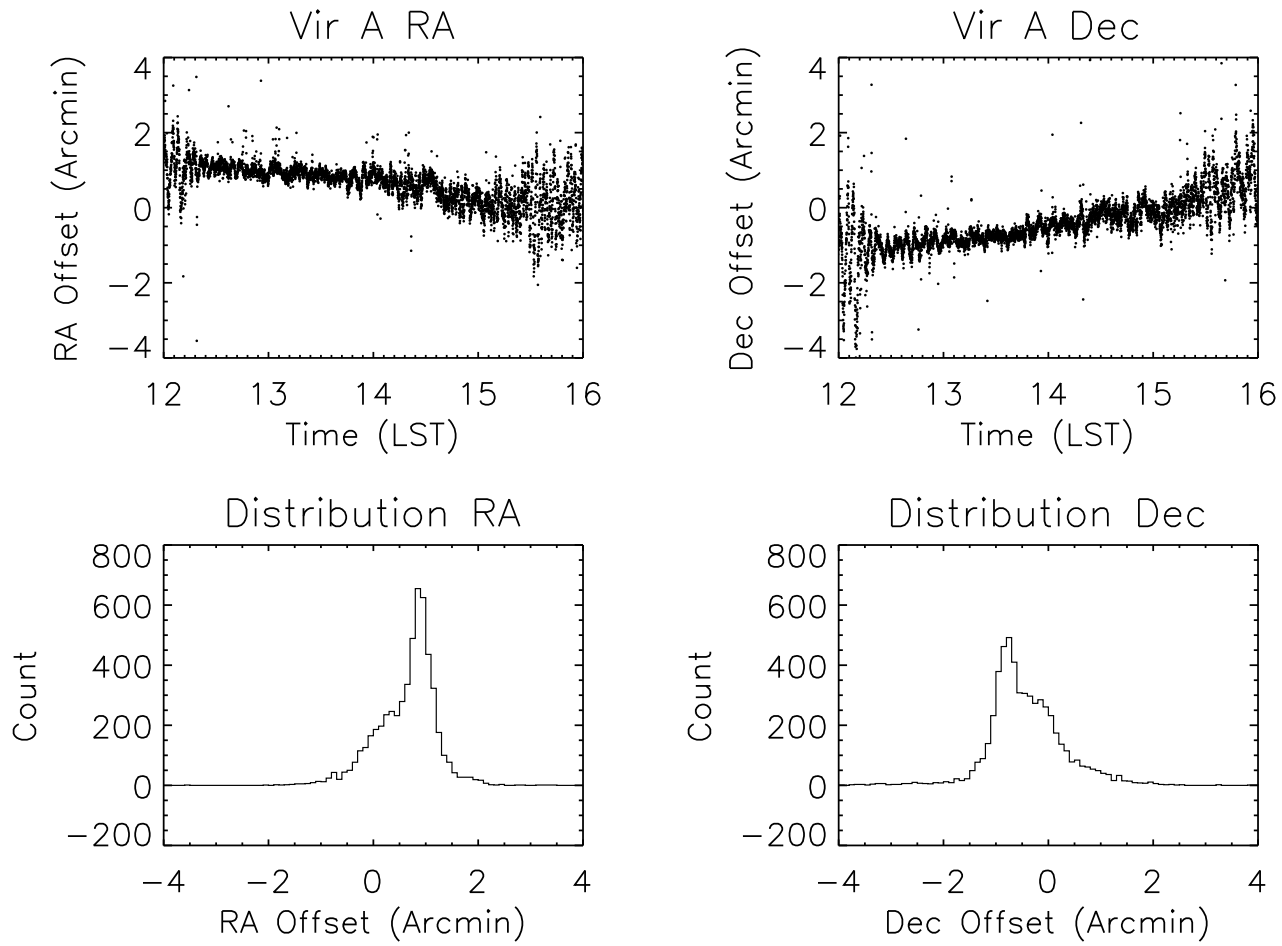

Fig. 4.9.- Top: Position offset in arcminutes vs local sidereal time for Vir A at $130 \mathrm{MHz}$, as measured by the process described in Section 4.3. Bottom: Histogram of these offsets. 

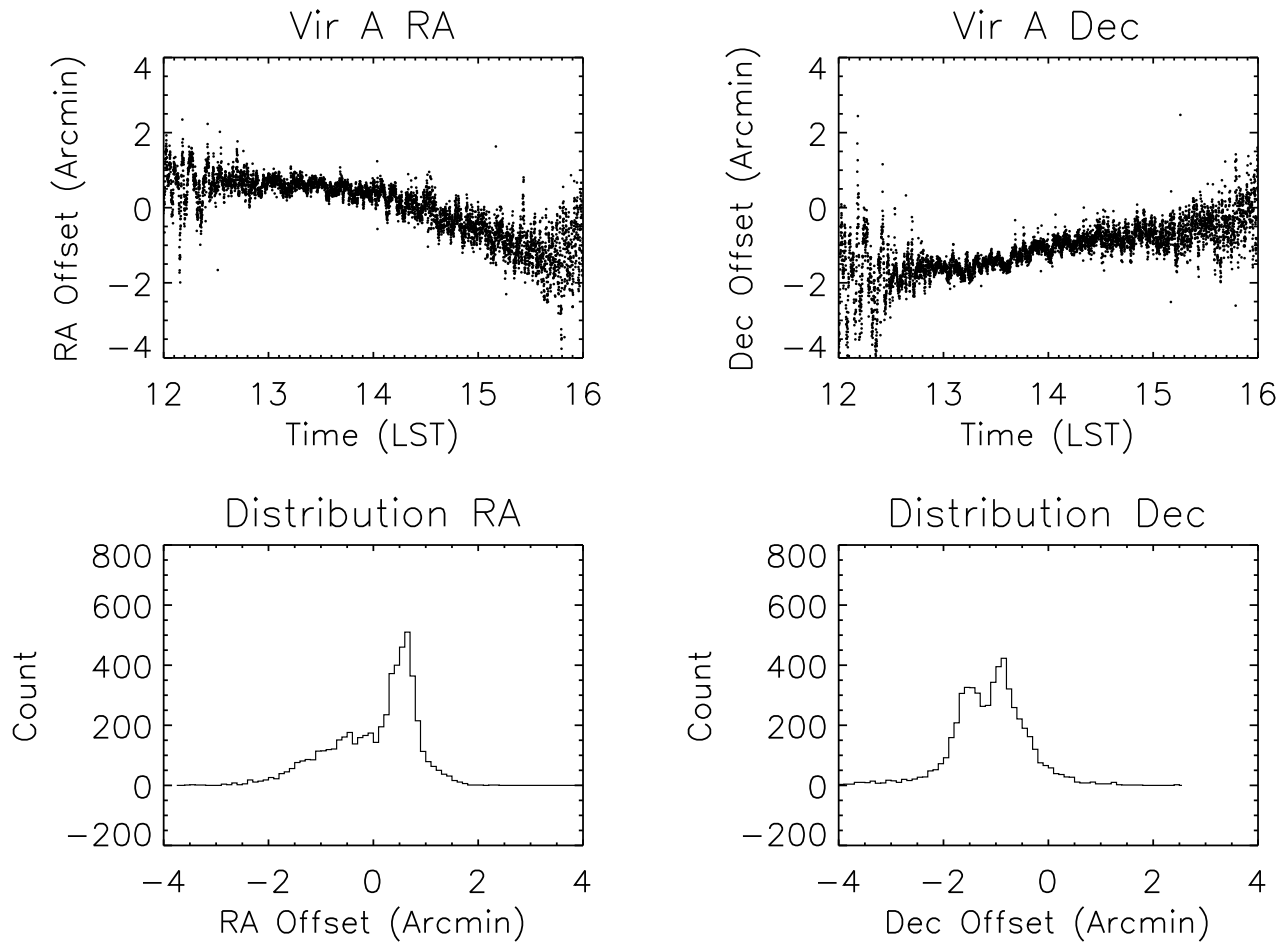

Fig. 4.10.- Top: Position offset in arcminutes vs local sidereal time for Vir A at $165 \mathrm{MHz}$, as measured by the process described in Section 4.3. Bottom: Histogram of these offsets. 

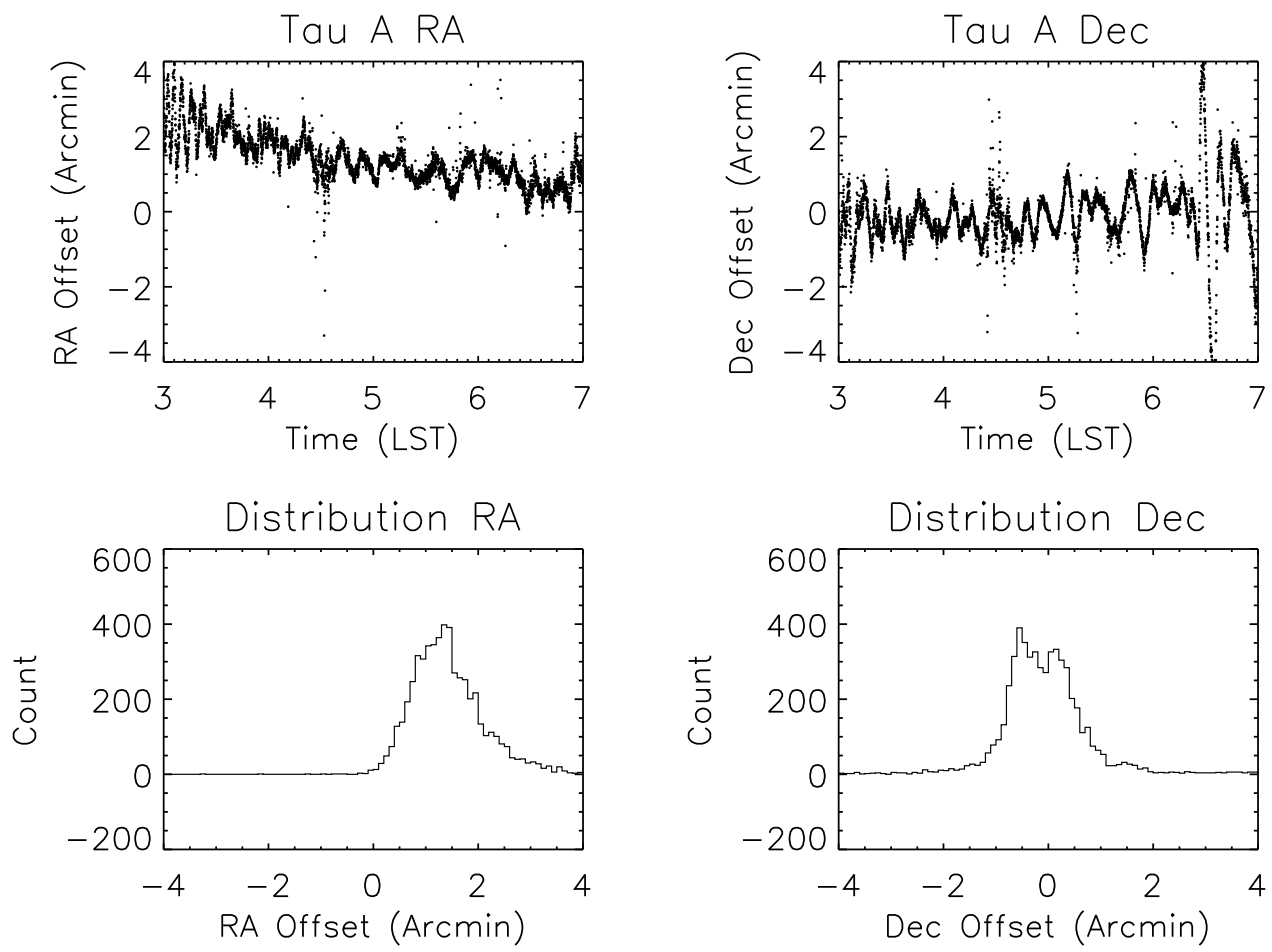

Fig. 4.11.- Top: Position offset in arcminutes vs local sidereal time for Tau A at $130 \mathrm{MHz}$, as measured by the process described in Section 4.3. Bottom: Histogram of these offsets. 

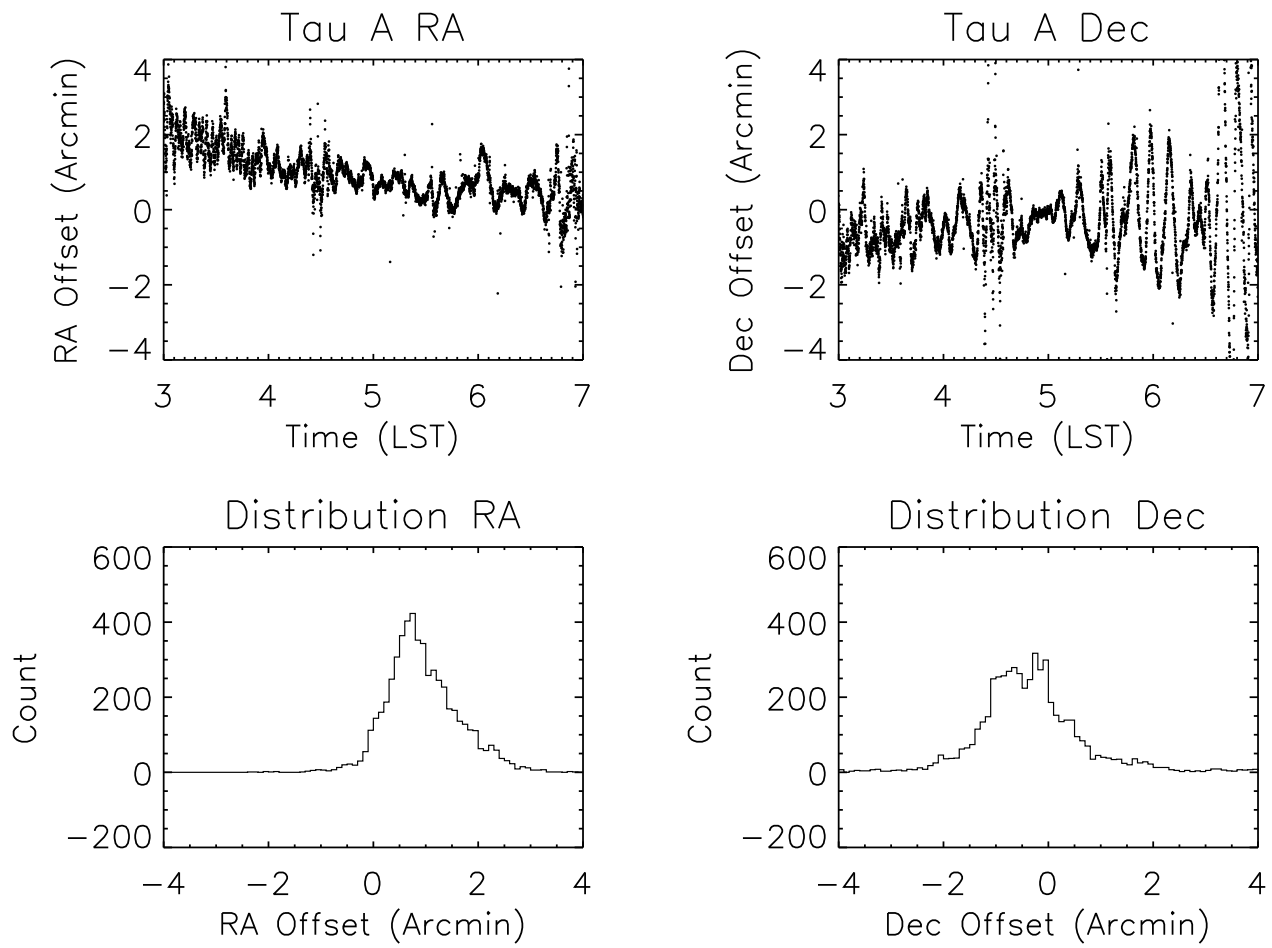

Fig. 4.12.- Top: Position offset in arcminutes vs local sidereal time for Tau A at $165 \mathrm{MHz}$, as measured by the process described in Section 4.3. Bottom: Histogram of these offsets. 
The offsets are expected to have a $\nu^{-2}$ dependence, so this is taken into account as a multiplicative offset before plotting and measuring the distributions. Median offsets are on the order of one arcminute, and the standard deviations of the offsets range from 0.08 arcminc to 1.61 arcmin. The former represents the overall refraction of the source due to the refractive effects of the ionosphere, and the standard deviation probes the scales of the irregularities. If these position offsets are due to a gradient in the total electron content as described in Eqn. 4.2, then these are estimated at $3 \times 10^{-2}$ to $4 \times 10^{-3}$ TECU km${ }^{-1}$. The distributions are not truly Gaussian and often are skewed by a few larger offsets.

These positions offsets are compared to the intrinsic source size at these wavelengths. Though these are all unresolved by PAPER, higher resolution images have been made with the VLA at $74 \mathrm{MHz}$. For Cyg A, Cas A, Tau A, and Vir A, highquality models have been created to help with calibrating data for other sources ${ }^{3}$. These are used as an approximation for intrinsic source size, and expect the positionfitting using the measurement equation to not do better than the intrinsic source size, regardless of signal-to-noise ratio.

For Cyg A and Vir A, the VLA models have been fit with elliptical Gaussians in CASA using the task "imfit." Measuring the distance between the half-power points of these along the longest axis gives an estimated source size of 2.5 arcminutes for Cyg A and 1.2 arcminutes for Vir A.

The structure of Cas A is more complicated, therefore it is estimated by measuring the width of the remnant from the contour levels that are $3 \sigma$ above the noise. With this, the source size is estimated to be just under 6 arcminutes. For Tau A, the emission is dominated by the central source at the position of the Crab pulsar, which is unresolved in the VLA data. However, using the same contour method as above,

\footnotetext{
${ }^{3}$ http://lwa.nrl.navy.mil/tutorial/
} 
the surrounding nebula is 8 arcminutes at its widest.

These rough source estimates could effect the precision to which the offsets can be measured, especially for a double-lobed source such as Cyg A, though there does not seem to be evidence of that. More important is the sensitivity of the interferometer. This can be estimated using

$$
S_{r m s}=\frac{2 k T_{s y s}}{A_{e f f} \sqrt{B t N(N-1) / 2}},
$$

were $k$ is Boltzmann's constant, $T_{\text {sys }}$ the system temperature, estimated to be $500 \mathrm{~K}$ when including the receiver temperature and the projected sky temperature,

$A_{e f f}$ is the effective area which from antenna model averages is estimated to be $3.7 \mathrm{~m}^{2}$, $B$ is the bandwidth, approximately $10 \mathrm{MHz}$ in these data, $t$ is the integration time of $10 \mathrm{~s}$, and $\mathrm{N}$ is the number of antennas, which is 32 in this case. This indicates a single snapshot rms flux of $\approx 50 \mathrm{mJy}$. Actual measurements of the image rms using CASA are closer to $100 \mathrm{mJy}$.

However, this method does not use imaging, but it uses the DDR filtering process described in the previous section. This is affected by the accuracy calibration to this point, since the sidelobes of the other large sources can confuse the process. Therefore, this process works best when the target source is high in the antenna primary beam and the other bright sources are below or near the horizon. For this reason, the plots in Fig. 4.5-4.12 show this "good" window of time around source transit.

\subsubsection{Comparison to Ionosphere Model}

These results are compared to a model screen ionosphere using a 2-D Kolmogorev spectrum screen as described by Martí-Vidal et al. (2010). A Kolmogorev screen assumes small scale, isotropic, self-similar turbulent motions, and is often used to 
Table 4.2: Source Position Offsets in Arcmins

\begin{tabular}{lcccccc}
\hline Source & Freq $(\mathrm{MHz})$ & Median & Std Dev & Points & Skew & Kurt \\
\hline Cas A & 130 & 0.84 & 0.65 & 13411 & 0.37 & -1.2 \\
Cas A & 165 & 0.95 & 0.98 & 13411 & 0.17 & -0.31 \\
Cyg A & 130 & 0.67 & 0.42 & 14290 & 5.9 & 176 \\
Cyg A & 165 & 0.63 & 0.50 & 14256 & 6.7 & 223 \\
Vir A & 130 & 1.08 & 0.85 & 5504 & 17 & 567 \\
Vir A & 165 & 1.43 & 0.60 & 5504 & 1.5 & 5.9 \\
Tau A & 130 & 1.54 & 1.04 & 5488 & 11 & 240 \\
Tau A & 165 & 1.26 & 1.89 & 5488 & 11 & 177 \\
\hline
\end{tabular}

a.

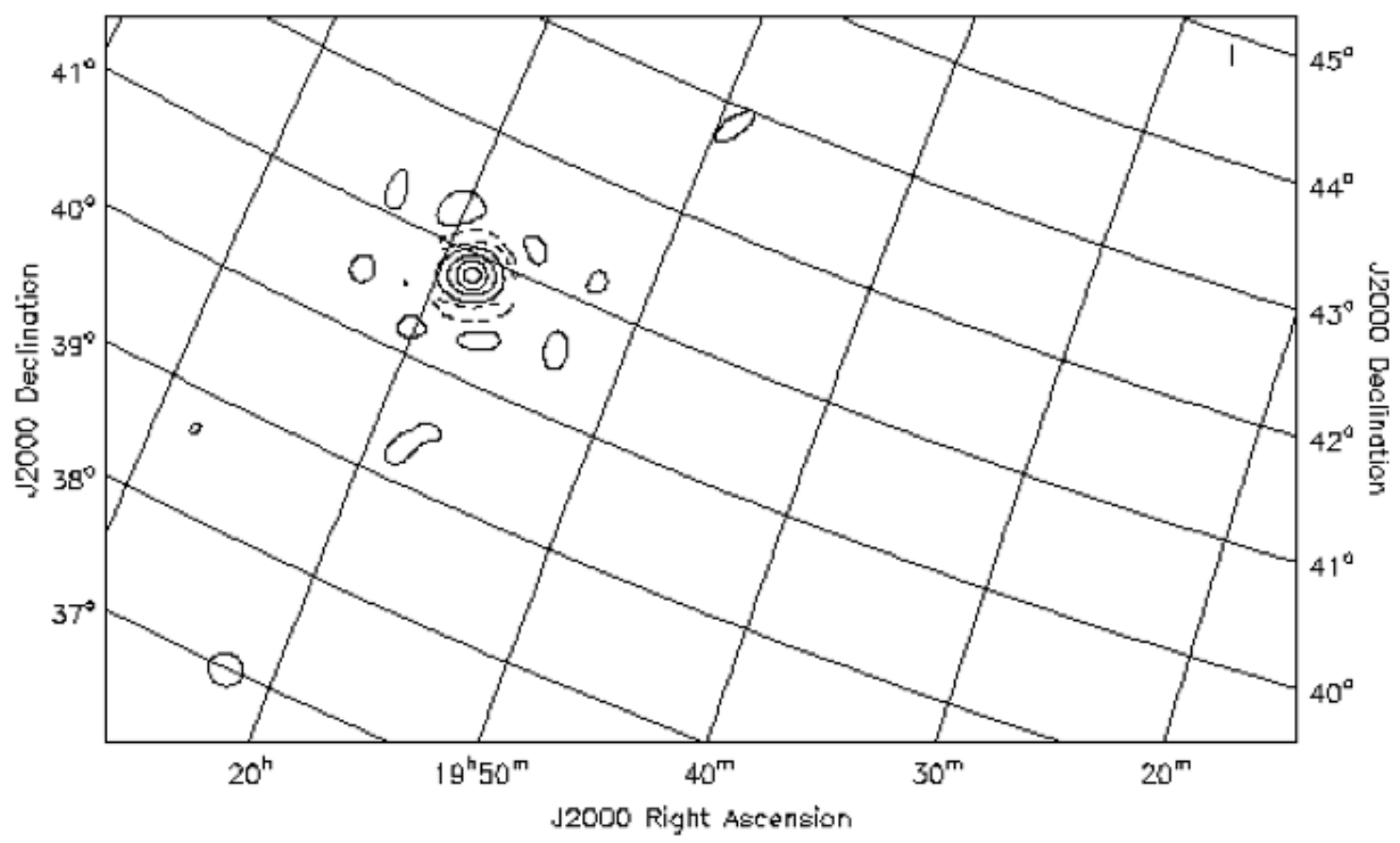

Fig. 4.13. - Contour plot of Cygnus A made with CASA. Cyg A is an unresolved point source as seen by PAPER, and image rms 100 mJy. 
describe a phase screen for optical astronomical observations. The standard model assumes a thin screen at a height of $300 \mathrm{~km}$ and a Fried length of $3 \mathrm{~km}$. The Fried length is the typical size scale over which the phase varies by less than a radian. It describes the size of the isoplanatic patch. This Fried length corresponds to an angular size scale of $\sim 0.82$ degrees. The standard model and resulting positions offsets from 2000 Monte-Carlo simulations are shown in Figure 4.14. These produce offsets that are several times larger than what is measured above. According to this model, the Fried parameter would have to be much larger, near $10 \mathrm{~km}$, to describe the observations using this model.

\subsection{Conclusion}

The first probe of the ionosphere using bright source position perturbations from the Precision Array for Probing the Epoch of Reionization (PAPER) are presented at 130 and $165 \mathrm{MHz}$. Using the brightest sources in the sky, source positions are fit using the visibility data and interferometer measurement equation. Short-term density fluctuations are detected at the current level of precision. Such effects of the ionosphere have plagued low-frequency astronomy for decades and will continue to provide a challenge for current and future telescopes searching for the signal of the Epoch of Reionization. A characterization of these density fluctuations will aid further imaging and analysis.

Position offsets showed perturbations due to the ionosphere on the scale of one or two arcminutes, and the distribution of offsets indicates that fluctuations are on the scale of 0.1 arcminutes. However, there exists significant scatter when comparing the frequency dependence across the PAPER band. These data show offsets an order of magnitude smaller than what is predicted from a two-dimensional Kolmogorev 


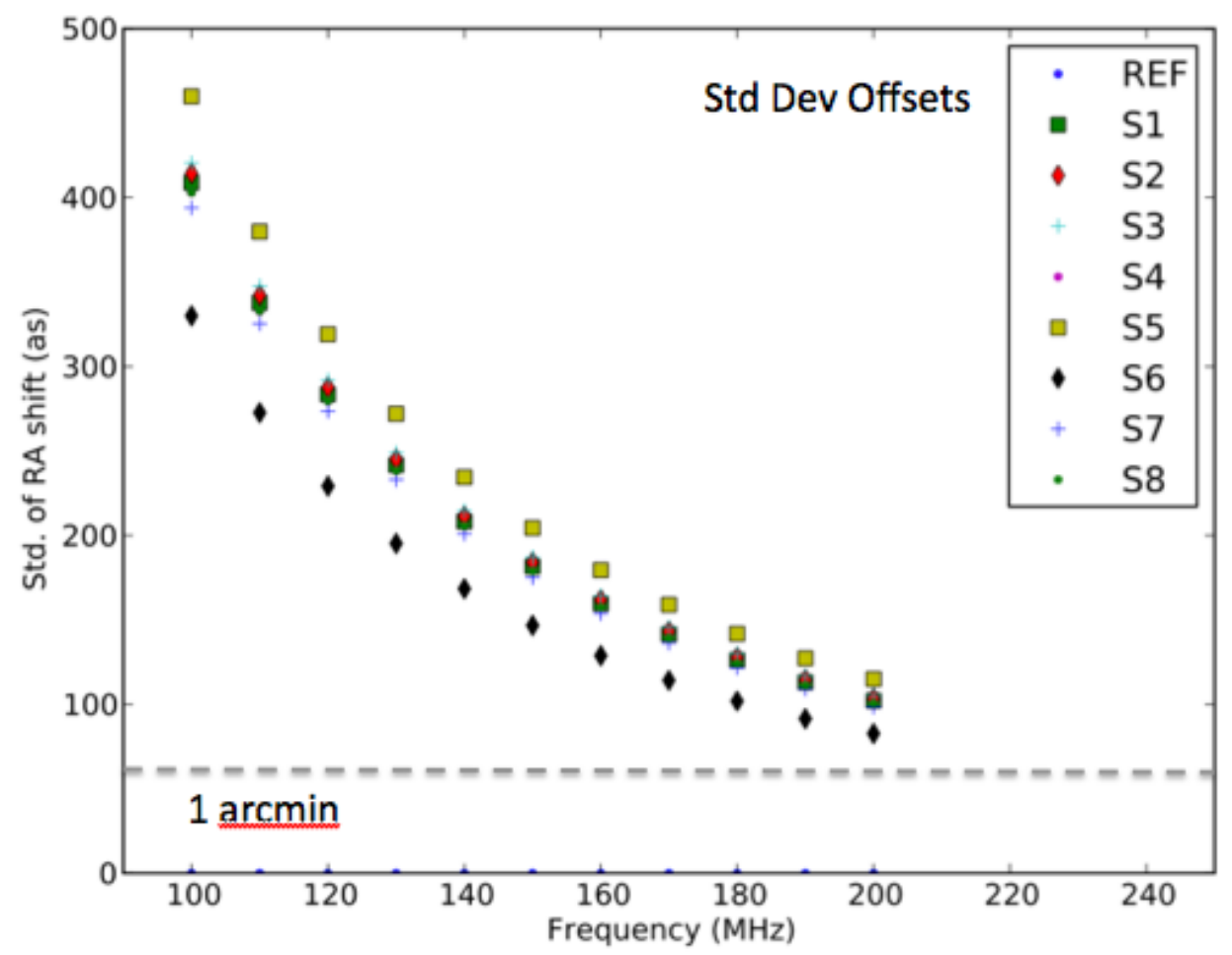

Fig. 4.14. - Results from Monte-Carlo simulations with a PAPER-like array and nine point sources using a 2-D Kolmogorev screen with a Fried length of $3 \mathrm{~km}$ at height of $300 \mathrm{~km}$. The standard deviation of position offsets are several times larger than the measured offsets in the PAPER data. 
spectrum phase screen model of the ionosphere with a Fried length parameter of $3 \mathrm{~km}$, or 0.8 degrees. This is encouraging in light of future observations to be done at these frequencies, particularly the Epoch of Reionization measurement that will require precision measurements of foreground sources to be removed.

The delay/delay-rate method of filtering bright sources from visibility data is effective for only the brightest radio sources in the PAPER data. Snapshot images made with the 32-element array do show a high signal-to-noise ratio, and thus further studies of the ionosphere with PAPER will use these images to look for ionosphere density fluctuations on the sky. PAPER's instantaneous wide field-of-view and wide bandwidth will be crucial to identifying large perturbations and determining ionospheric disturbances by their frequency dependance. 


\section{Chapter 5}

\section{General Conclusions}

PAPER has grown from a tiny underdog of a project to a serious competitor for being the first to detect the power spectrum of the Epoch of rReionization. By following a philosophy of small changes and progressively larger deployments, it has become a sensitive, stable radio telescope with an end-to-end system of deployment without spending much on early infrastructure costs. PAPER has the advantage of a clean primary beam and well-characterized RF system to aid in the calibration needed to detect the sensitive EoR signal. The path ahead, however, is not easy.

The stability of PAPER's RF amplifiers is affected largely by the ambient temperature of the cables and balun that are exposed to the elements and to a smaller extent by the receivers which are kept in a climate-controlled space. Taking these terms into account significantly helps in making accurate measurements of the sky temperature, as evidenced by single-antenna measurements of the total power, dominated by the Galactic synchrotron.

This work presents the first probe of the ionosphere using bright source position perturbations from PAPER at around $150 \mathrm{MHz}$. Using the brightest sources in the sky, source positions are fit using the visibility data and interferometer measurement 
equation. Short-term density fluctuations are not detected with a level of precision of several arcminutes. Such effects of the ionosphere have plagued low-frequency astronomy for decades and will continue to provide a challenge for current and future telescopes searching for the signal of the EoR. A characterization of these density fluctuations will aid further imaging and analysis.

The delay/delay-rate method of filtering bright sources from visibility data is effective for only the brightest radio sources in the PAPER data. Snapshot images made with the 32-element array do show a high signal-to-noise ratio, and thus further studies of the ionosphere with PAPER will use these images to look for ionospheric density fluctuations on the sky. PAPER's instantaneous wide field-of-view and wide bandwidth will be crucial to identifying large perturbations and determining ionospheric disturbances by their frequency dependance.

Initial probes of the ionosphere as seen by PAPER demonstrate that bright sources are refracted by a few arcminutes over timescales measured in minutes. This is comparable to a Kolmogorev spectrum for the disturbances in the ionosphere with a larger isoplanatic patch than predicted, when compared to a simple, 2D model of the ionosphere. This is a factor of 10 better than the fiducial value and thus indicates that the problem of the ionosphere will be a solvable one for spatially-small low-frequency arrays in the future.

Datta et al. (2009) investigated the kind of stability and precision needed by a PAPER-like array in order to detect the power spectrum of reionization. The desired RMS noise level in their simulations requires 0.1 arcsecond position accuracy and residual calibration errors of $<0.2 \%$. However, these constraints are on detection in the imaging domain. As expected, PAPER and other concurrent instruments are likely several orders of magnitude away from being able to detect the EoR from 
imaging.

For future investigations, the 64-antenna array in South Africa has been reconfigured to a maximum redundancy array. That is, the antennas are arranged in rows such that a few antenna spacings are sampled multiple times with the idea of increasing sensitivity for certain spatial frequencies (Parsons et al 2011). Results are forthcoming. The next 64-elements of PAPER-SA are currently under construction to be deployed before the end of 2012. Then, an attempt at detecting the EoR can be made. 


\section{References}

Backer, D. C., Aguirre, J., Bowman, J. D., Bradley, R., Carilli, C. L., Furlanetto, S. R., Greenhill, L. J., Hewitt, J. N., Lonsdale, C., Ord, S. M., Parsons, A., \& Whitney, A. 2010, White paper for the Astro2010 Decadal Survey

Barkana, R. 2009, MNRAS, 397, 1454

Becker, R. H., Fan, X., White, R. L., Strauss, M. A., Narayanan, V. K., Lupton, R. H., Gunn, J. E., Annis, J., Bahcall, N. A., Brinkmann, J., Connolly, A. J., Csabai, I., Czarapata, P. C., Doi, M., Heckman, T. M., Hennessy, G. S., Ivezić, Ž., Knapp, G. R., Lamb, D. Q., McKay, T. A., Munn, J. A., Nash, T., Nichol, R., Pier, J. R., Richards, G. T., Schneider, D. P., Stoughton, C., Szalay, A. S., Thakar, A. R., \& York, D. G. 2001, AJ, 122, 2850

Bowman, J. D. \& Rogers, A. E. E. 2010, Nature, 468, 796

Bradley, R. F. 2006, NRAO Green Bank Electronics Division Internal Reports

Bridle, A. H. 1967, MNRAS, 136, 219

Clark, B. G. 1999, in Astronomical Society of the Pacific Conference Series, Vol. 180, Synthesis Imaging in Radio Astronomy II, ed. G. B. Taylor, C. L. Carilli, \& R. A. Perley, 1 
Cohen, A. S., Lane, W. M., Cotton, W. D., Kassim, N. E., Lazio, T. J. W., Perley, R. A., Condon, J. J., \& Erickson, W. C. 2007, AJ, 134, 1245

Cohen, A. S. \& Rottgering, H. J. A. 2009, AJ, 138, 439

Condon, J. J., Cotton, W. D., Greisen, E. W., Yin, Q. F., Perley, R. A., Taylor, G. B., \& Broderick, J. J. 1998, AJ, 115, 1693

Condon, J. J. \& Ransom, S. 2007, Online Lecture Notes

Erickson, W. C., Perley, R. A., Flatters, C., \& Kassim, N. E. 2001, A\&A, 366, 1071

Fan, X., Strauss, M. A., Becker, R. H., White, R. L., Gunn, J. E., Knapp, G. R., Richards, G. T., Schneider, D. P., Brinkmann, J., \& Fukugita, M. 2006, AJ, 132, 117

Fan, X., Strauss, M. A., Becker, R. H., White, R. L., Gunn, J. E., Knapp, G. R., Richards, G. T., Schneider, D. P., Brinkmann, J., \& Fukugita, M. 2006, AJ, 132, 117

Field, G. B. 1959, ApJ, 129, 536

Furlanetto, S. R., Oh, S. P., \& Briggs, F. H. 2006, Phys. Rep., 433, 181

Furlanetto, S. R., Sokasian, A., \& Hernquist, L. 2004a, MNRAS, 347, 187

Furlanetto, S. R., Zaldarriaga, M., \& Hernquist, L. 2004b, ApJ, 613, 1

Gunn, J. E. \& Peterson, B. A. 1965, ApJ, 142, 1633

Hinder, R. \& Ryle, M. 1971, MNRAS, 154

Hogbom, J. A. 1974, A\&AS, 15 
Intema, H. T., van der Tol, S., Cotton, W. D., Cohen, A. S., van Bemmel, I. M., \& Rottgering, H. J. A. 2009, A\&A, 501, 1185

Kassim, N. E., Lazio, T. J. W., Erickson, W. C., Perley, R. A., Cotton, W. D., Greisen, E. W., Cohen, A. S., Hicks, B., Schmitt, H. R., \& Katz, D. 2007, ApJS, 172,686

Kassim, N. E., Perley, R. A., Erickson, W. C., \& Dwarakanath, K. S. 1993, AJ, 106, 2218

Kellermann, K. I., Pauliny-Toth, I. I. K., \& Williams, P. J. S. 1969, ApJ, 157

Kraus, J. D. 1966, Radio astronomy (New York: McGraw-Hill)

Larson, D., Dunkley, J., Hinshaw, G., Komatsu, E., Nolta, M. R., Bennett, C. L., Gold, B., Halpern, M., Hill, R. S., Jarosik, N., Kogut, A., Limon, M., Meyer, S. S., Odegard, N., Page, L., Smith, K. M., Spergel, D. N., Tucker, G. S., Weiland, J. L., Wollack, E., \& Wright, E. L. 2011, ApJS, 192

Lawson, K. D., Mayer, C. J., Osborne, J. L., \& Parkinson, M. L. 1987, MNRAS, 225, 307

Lonsdale, C. J. 2005, ASPC, 345

Lonsdale, C. J., Cappallo, R. J., Morales, M. F., Briggs, F. H., Benkevitch, L., Bowman, J. D., Bunton, J. D., Burns, S., Corey, B. E., Desouza, L., Doeleman, S. S., Derome, M., Deshpande, A., Gopala, M. R., Greenhill, L. J., Herne, D. E., Hewitt, J. N., Kamini, P. A., Kasper, J. C., Kincaid, B. B., Kocz, J., Kowald, E., Kratzenberg, E., Kumar, D., Lynch, M. J., Madhavi, S., Matejek, M., Mitchell, D. A., Morgan, E., Oberoi, D., Ord, S., Pathikulangara, J., Prabu, T., Rogers, A., 
Roshi, A., Salah, J. E., Sault, R. J., Shankar, N. U., Srivani, K. S., Stevens, J., Tingay, S., Vaccarella, A., Waterson, M., Wayth, R. B., Webster, R. L., Whitney, A. R., Williams, A., \& Williams, C. 2009, Proceedings of the IEEE, 97, 1497

Martí-Vidal, I., Guirado, J. C., Jiménez-Monferrer, S., \& Marcaide, J. M. 2010, A\&A, $517, \mathrm{~A} 70$

McQuinn, M., Zahn, O., Zaldarriaga, M., Hernquist, L., \& Furlanetto, S. R. 2006, ApJ, 653, 815

Mitra, S., Choudhury, T. R., \& Ferrara, A. 2011, MNRAS, 413, 1569

Morales, M. F. \& Wyithe, J. S. B. 2010, ARA\&A, 48, 127

Mortonson, M. J. \& Hu, W. 2008, ApJ, 686, L53

Paciga, G., Chang, T.-C., Gupta, Y., Nityanada, R., Odegova, J., Pen, U.-L., Peterson, J. B., Roy, J., \& Sigurdson, K. 2011, MNRAS, 413, 1174

Parashare, C. 2011, Ph.d thesis, University of Virginia

Parsons, A. R. \& Backer, D. C. 2009, AJ, 138, 219

Parsons, A. R., Backer, D. C., Foster, G. S., Wright, M. C. H., Bradley, R. F., Gugliucci, N. E., Parashare, C. R., Benoit, E. E., Aguirre, J. E., Jacobs, D. C., Carilli, C. L., Herne, D., Lynch, M. J., Manley, J. R., \& Werthimer, D. J. 2010, AJ, 139, 1468

Perley, R. A. \& Erickson, W. C. 1984, NRAO VLA Scientific Memos

Ratcliffe, J. A. 1972, An Introduction to the Ionosphere and Magnetosphere (New York: Cambridge University Press) 
Roger, R. S., Costain, C. H., Landecker, T. L., \& Swerdlyk, C. M. 1999, A\&AS, 137, 7

Rogers, A. E. E. 2004, Deuterium Array Memo No. 10

Rottgering, H. J. A., Braun, R., Barthel, P. D., van Haarlem, M. P., Miley, G. K., Morganti, R., Snellen, I., Falcke, H., de Bruyn, A. G., Stappers, R. B., Boland, W. H. W. M., Butcher, H. R., de Geus, E. J., Koopmans, L., Fender, R., Kuijpers, J., Schilizzi, R. T., Vogt, C., Wijers, R. A. M. J., Wise, M., Brouw, W. N., Hamaker, J. P., Noordam, J. E., Oosterloo, T., Bahren, L., Brentjens, M. A., Wijnholds, S. J., Bregman, J. D., van Cappellen, W. A., Gunst, A. W., Kant, G. W., Reitsma, J., van der Schaaf, K., \& de Vos, C. M. 2006, ArXiv Astrophysics e-prints

Santos, M. G., Cooray, A., \& Knox, L. 2005, ApJ, 625, 575

Seager, S., Sasselov, D. D., \& Scott, D. 1999, ApJ, 523, L1

Sheth, R. K. 1998, MNRAS, 300, 1057

Sironi, G. 1974, MNRAS, 166, 345

Sokasian, A., Abel, T., Hernquist, L., \& Springel, V. 2003, MNRAS, 344, 607

Spoelstra, T. A. T. 1983, AAP, 120, 313

Springel, V. \& Hernquist, L. 2003, MNRAS, 339, 312

Springel, V., White, S. D. M., Jenkins, A., Frenk, C. S., Yoshida, N., Gao, L., Navarro, J., Thacker, R., Croton, D., Helly, J., Peacock, J. A., Cole, S., Thomas, P., Couchman, H., Evrard, A., Colberg, J., \& Pearce, F. 2005, Nature, 435, 629

Wouthuysen, S. A. 1952, AJ, 57, 31 
Wrobel, J. M. \& Walker, R. C. 1999, in Astronomical Society of the Pacific Conference Series, Vol. 180, Synthesis Imaging in Radio Astronomy II, ed. G. B. Taylor, C. L. Carilli, \& R. A. Perley, 171 